\title{
Reflex or reflect? Implicit and explicit cognitive processes and early intervention in adolescent alcohol use
}

Citation for published version (APA):

Thush, C. (2008). Reflex or reflect? Implicit and explicit cognitive processes and early intervention in adolescent alcohol use. [Doctoral Thesis, Maastricht University]. Datawyse / Universitaire Pers Maastricht. https://doi.org/10.26481/dis.20081009ct

Document status and date:

Published: 01/01/2008

DOI:

10.26481/dis.20081009ct

Document Version:

Publisher's PDF, also known as Version of record

\section{Please check the document version of this publication:}

- A submitted manuscript is the version of the article upon submission and before peer-review. There can be important differences between the submitted version and the official published version of record.

People interested in the research are advised to contact the author for the final version of the publication, or visit the DOI to the publisher's website.

- The final author version and the galley proof are versions of the publication after peer review.

- The final published version features the final layout of the paper including the volume, issue and page numbers.

Link to publication

\footnotetext{
General rights rights.

- You may freely distribute the URL identifying the publication in the public portal. please follow below link for the End User Agreement:

www.umlib.nl/taverne-license

Take down policy

If you believe that this document breaches copyright please contact us at:

repository@maastrichtuniversity.nl

providing details and we will investigate your claim.
}

Copyright and moral rights for the publications made accessible in the public portal are retained by the authors and/or other copyright owners and it is a condition of accessing publications that users recognise and abide by the legal requirements associated with these

- Users may download and print one copy of any publication from the public portal for the purpose of private study or research.

- You may not further distribute the material or use it for any profit-making activity or commercial gain

If the publication is distributed under the terms of Article 25fa of the Dutch Copyright Act, indicated by the "Taverne" license above, 
Reflex or Reflect?

Implicit and Explicit Cognitive Processes and Early Intervention in Adolescent Alcohol Use 


\section{Colophon}

ISBN: 9789052787572

Graphic design: Carolien Thush

Production: Datawyse, Universitaire Pers Maastricht

@ Carolien Thush, Maastricht 2008

The studies presented in this dissertation were funded by Dutch Health Care Research Organization (ZonMw; 2100.0058 and 31000065) and the National Institute on Drug Abuse (NIDA; DA16094). 


\title{
Reflex or Reflect? \\ Implicit and Explicit Cognitive Processes and Early Intervention in Adolescent Alcohol Use
}

\begin{abstract}
PROEFSCHRIFT
Ter verkrijging van de graad van doctor aan de Universiteit Maastricht, op gezag van de Rector Magnificus, prof. mr. G.P.M.F. Mols, volgens het besluit van het College van Decanen, in het openbaar te verdedigen op donderdag

9 oktober 2008 om 12.00 uur

door

Carolien Thush
\end{abstract}

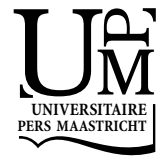




\section{Promotores}

Prof. dr. A. T. M. Janssen

Prof. dr. R.W. Wiers

\section{Beoordelingscomissie}

Prof. dr. R. A. Knibbe (voorzitter)

Prof. dr. R. C. M. E. Engels (Radboud Universiteit Nijmegen)

Prof. dr. J. J. Jolles

Prof. dr. M. L. Peters

Prof. dr. W. A. M. Vollebergh (Universiteit Utrecht) 


\section{Contents}

$\begin{array}{lll}\text { Chapter } 1 \text { General Introduction } & 7\end{array}$

Chapter 2 A randomized clinical trial of a targeted intervention to moderate 21 alcohol use and alcohol-related problems in at-risk adolescents

Chapter 3 Explicit and implicit alcohol-related cognitions and the prediction of 39 future drinking in adolescents

Chapter 4 Apples and oranges? Comparing indirect measures of alcohol-related 59 cognition predicting alcohol use in at-risk adolescents

Chapter 5 Interactions between implicit and explicit cognition and working 73 memory capacity in the prediction of alcohol use in at-risk adolescents

Chapter 6 The influence of Motivational Interviewing on explicit and implicit 93 alcohol-related cognition and alcohol use in at-risk adolescents

Chapter 7 General Discussion

Summary

Samenvatting

References

Dankwoord 



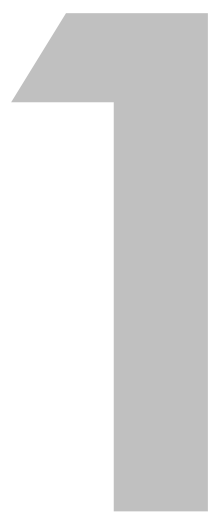

General Introduction 


\section{ADOLESCENTS AT RISK?}

Adolescence is not only a developmental period in which body and brain mature, but it is also a period which offers other special opportunities as well as vulnerabilities (Dahl, 2004). Due to the plasticity of the adolescent brain, opportunity arises for reasoning capabilities and decision making skills to develop at a rapid pace. Nevertheless, this plasticity of the adolescent brain has a downside as well: emotional deregulation.

This emotional instability has been explained by the different pace at which several brain, behavioral and cognitive systems mature. For instance, while sensation seeking and emotional arousability peaks already in early adolescence, fully coordinated executive functioning does not develop until late adolescence or young adulthood (e.g. Luna, 2004; Steinberg, 2005). As such, adolescents are relative good at making rationally sound decisions under conditions of low arousal ('cool processing'), yet they have great difficulty coming to a responsible rational choice under conditions of high arousal and strong emotions ('hot processing') (Dahl, 2004). For example, adolescents tend to exhibit more risky behavior in the presence of peers than alone compared to adults (Gardner \& Steinberg, 2005). This demonstrates that adolescents are not merely incapable of making rational sound decisions, but that under certain conditions of high arousal cross-talk between the emotional and cognitive control system is impaired (Steinberg, 2007).

Fortunately, although adolescence is a turbulent period, most adolescents do find their way into adulthood without too many problems (Arnett, 1999). Adolescents seem to grow out of certain types of risky behaviors, such as excessive alcohol use, when taking on new responsibilities and more adult roles (e.g. Newcomb \& Bentler, 1987; Sher \& Gotham, 1999). However, still a significant proportion of these adolescents do grow up struggling with emotional, cognitive and social troubles which shapes their trajectory into adulthood (Dahl, 2004). The use and misuse of alcohol can be seen as one of these problems, which can have great impact in adolescence and on later periods of life.

\section{ADOLESCENT ALCOHOL USE}

Adolescent alcohol abuse is a serious problem. Compared to adults relatively a large proportion of adolescents, namely $22 \%$, are problem drinkers (Van Dijck \& Knibbe, 2005). Already at the age of twelve, $30 \%$ of Dutch adolescents reports having consumed alcohol in the past month, and this number increases with age $143 \%$ of thirteen year olds, $62 \%$ of fourteen year olds, $70 \%$ of fifteen year-olds; Monshouwer, van Dorsselaer, Gorter, Verdurmen, \& Vollebergh, 2004). Dutch adolescents are among the most frequent binge drinkers in Europe (Hibell et al., 2004). Binge drinking can be defined as 
consuming large amounts of alcohol on one occasion'. Binge drinking often occurs during the weekend (Monshouwer et al., 2004) and on holidays (Van de Luitgaarden, Wiers, Knibbe, \& Boon, 2006). As a result, the number of alcohol poisonings among 12-17 year olds is rising (Van Kleef \& Van der Lely, 2006).

Research has shown that alcohol abuse among adolescents increases the probability of severe short term consequences like forced and unsafe sex, dropping out of school, unemployment, social isolation, and depression (e.g. Newcomb \& Bentler, 1989). Furthermore, alcohol abuse during adolescence has negative long term consequences. Research has indicated that an early onset of drinking during adolescence predicts alcohol-related problems later in life (Grant \& Dawson, 1997). Additionally, animal research has demonstrated that the long term effects of binge drinking on the adolescent brain differ and are more severe compared with the effects on the adult brain (e.g. Crews, Braun, Hoplight, Switzer, \& Knapp 2000; White et al., 2000; White \& Swartzwelder, 2004). Moreover, studies involving human adolescents have revealed that alcohol abuse has negative consequences for the maturation of brain regions (e.g., DeBellis et al., 2000), causes impaired neuropsychological functioning (e.g., Brown, Tapert, Granholm, \& Dellis, 2000) and altered processing in executive functioning tasks (Tapert et al., 2004). Thus, adolescents seem to be more sensitive than adults to alcohol-induced damage in structures, which play a role in the control of impulses that, for example, could prevent binge drinking episodes (Spear, 2004).

This all suggests that adolescence is a very important period to try to moderate hazardous adolescent drinking to safer levels. Not in the least because a strong base for problematic addictive behavior is (in retrospect) often found in adolescence (Grant \& Dawson, 1997). Besides the need for better Dutch government policy concerning adolescent drinking (Van de Luitgaarden, Thush, Wiers, \& Knibbe, 2008), it is also imperative to investigate the cognitive alcohol-related processes that guide adolescent drinking behavior. This can bring new theoretical insights into the processes of initiation and development of adolescents drinking. Additionally, this might have important implications for the development of intervention programs targeted at reducing adolescent drinking.

\footnotetext{
1 In the US the National Institute on Alcohol Abuse and Alcoholism (NIAAA) has classified binge drinking as an increase of the blood alcohol concentration (BAC) to 0.08 percent or more. For men, binge drinking can thus be defined as consuming five or more standard alcohol servings on one occasion and for women as consuming four more standard alcohol servings on one occasion. However, a standard alcohol serving in the Netherlands contains somewhat less alcohol than an American standard alcohol serving: approximately 10 vs. 14 gr. In the Netherlands, binge drinking is therefore often generally defined as drinking five or more standard alcohol servings on one occasion.
} 


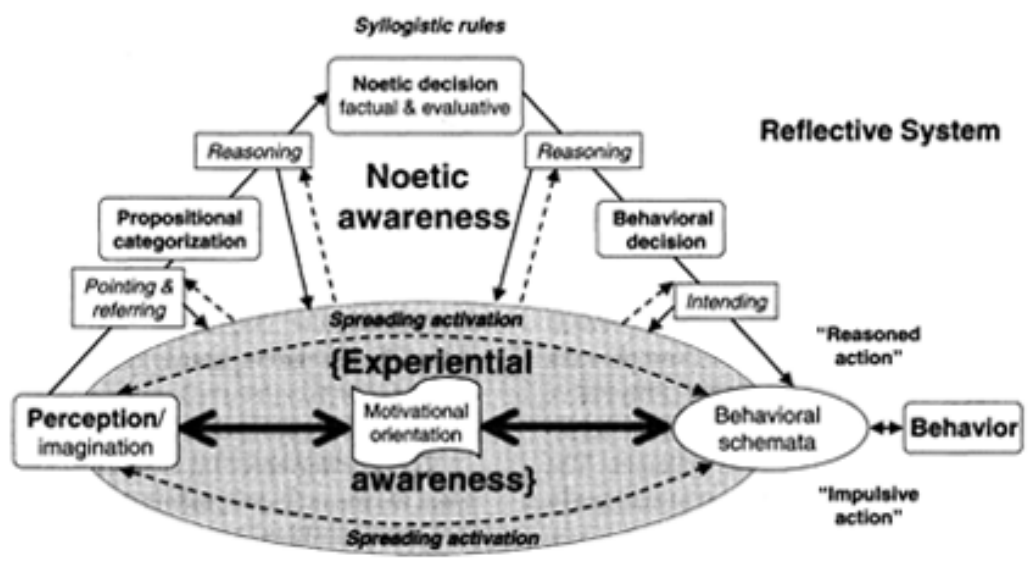

Associative store

Episodic and semantic links

Impulsive System

RS -

Figure 1. Reflective Impulsive Model by Strack and Deutsch (2004).

\section{ALCOHOL USE \& COGNITIVE PROCESSES}

Traditional theoretical models, such as the theory of planned behavior (Ajzen, 1991), explain the existence of maladaptive health-related behavior (like addictive behaviors) by the influence of distorted subjective norms, general attitudes and perceived selfefficacy in the rational decisional process. However, these types of theoretical models have difficulty explaining why people persist with maladaptive health-related behavior even when they are well aware of the risk factors involved and harmful consequences. The occurrence of maladaptive health-related behavior, addictive behavior specifically, might not always be based on a rational decision process. Accordingly, general cognitive dual process models predict that both more reflective explicit and more impulsive implicit cognitive processes influence behavior (e.g. Fazio \& Towles-Schwen, 1999; Kahneman, 2003; Strack \& Deutsch, 2004). Implicit cognitive processes represent more automatic associative motivational processes, whereas explicit cognitive processes are related to more deliberate thought processes (Greenwald \& Banaji, 1995; Kahneman, 2003; Strack \& Deutsch, 2004). Additionally, an assumption of these general dual process models is, that the influence of these implicit processes on subsequent behavior is moderated by explicit processes when a person is motivated enough and has the opportunity to do so (e.g. Fazio \& Towles-Schwen, 1999; Kahneman, 2003; Strack \& Deutsch, 2004). For example, according to the Reflective Impulsive Model (RIM; Strack \& Deutsch, 2004; see Figure 1), one can perform logical operations on the 
content of these impulsive implicit processes in the reflective explicit processing mode (such as negation and placing the content in a different time frame). Nevertheless, explicitly reversing an automatic association in a reflective mode of processing (negation) does not necessarily change the content of the impulsive implicit processes (Deutsch, Gawronski, \& Strack, 2006). In that respect these explicit and implicit processes influence behavior independently. However, under the right circumstances, these reflective operations can consequently lead to a diminished influence of the impulsive implicit processes on subsequent behavior.

Specifically, dual process models of addiction predict that the influence of implicit appetitive cognitive processes on subsequent addictive behavior might be moderated by explicit reflective cognitive processes when an individual's level of executive functioning and motivation is sufficient to do so (e.g. Stacy, Ames, \& Knowlton, 2004; Wiers et al, 2007; for example see Figure 2). Indeed, both implicit and explicit alcohol-related cognitions have been shown to predict a unique part of the variance in current and prospective adult alcohol use (e.g. Jajodia \& Earleywine, 2003; Stacy, 1997; Wiers, van Woerden, Smulders \& De Jong, 2002). Additionally, neurobiological addiction research has indeed shown that the prefrontal cortex and associated areas are not only involved in more reflective decision making, but also in the moderation of impulses to use substances (e.g. Bechara, 2005; Kalivas \& Volkow, 2005; Wilson, Sayette \& Fiez, 2004).

The unique influence of these implicit and explicit processes on drinking behavior can be explained by taking the concept of time into consideration. While immediate effects of consuming alcohol (often positive) will be stored as relatively automatic alcohol associations, more proximate effects of alcohol (often negative) require more reflection and will not immediately be stored as automatic associations. Consequently, both automatic appetitive alcohol associations triggered by alcohol-related cues and more deliberate inhibitory responses fueled by more distal negative outcomes of drinking will influence behavior through different processing modes. The assumption that alcohol and drug-related cues will automatically activate appetitive associations and more time is needed to reflect on the negative consequences of alcohol use is in line with the aspect of time presented in the model by Strack and Deutsch (2004).

In sum, according to dual process models of addictive behaviors both explicit and implicit alcohol-related cognitive processes influence the development of adolescent alcohol use. Explicit processes can moderate the influence of implicit processes, given that the level of executive functioning and motivation is sufficient. In the following paragraphs each of these elements will be discussed separately. In this general introduction and throughout this thesis, explicit and implicit cognitive processes, explicit and implicit alcohol-related cognition and explicit and implicit (or direct and indirect) measures will be used. The qualification 'processes' will be used to distinguish the manner in which alcohol-related information is processed or influences behavior. The term 'cognition' will be used when the content of these processes is discussed. The use of the terms explicit and implicit alcohol-related cognition does not directly imply that 
there are distinct implicit and explicit cognitions; these terms are used to indicate that alcohol-related cognition can be measured with direct or indirect measures and the outcomes of these measures influences behavior through different pathways. The terms direct and indirect (or implicit and explicit) measures will be used to differentiate between procedures that measure alcohol-related cognition in a way that resembles how explicit and implicit processes work (De Houwer, 2006)

\section{Explicit Alcohol-Related Cognition}

Explicit alcohol-related cognitive processes have been investigated in many studies on alcohol outcome expectancies. Explicit alcohol outcome expectancies can be defined as propositional if-then beliefs an individual has about the effect of drinking alcohol (Goldman, Del Boca \& Darkes, 1999). For example, early (direct or vicarious) experiences with alcohol may result in that behavior (e.g. drinking alcohol) and affecttive outcomes (e.g. excitement) become causally connected in memory. This affective aspect of explicit alcohol outcome expectancies can be placed along different dimensions: a positive-negative dimension (valence) and an arousal-sedation dimension (activation). The most common alcohol expectancies can be classified as positive arousal, positive sedation or negative alcohol expectancies (Goldman et al. 1999; Goldman \& Darkes, 2004). Once these expectancies are formed, an individual can decide to engage (or not to engage) in the drinking of alcohol based on what is expected as a result (e.g. 'alcohol makes me feel excited').

Explicit alcohol outcome expectancies have shown to be strongly correlated with current alcohol use, predicting up to half of the variance in concurrent alcohol use in cross-sectional studies (Goldman et al., 1999; Wiers, Hoogeveen, Sergeant \& Gunning, 1997). Explicit alcohol outcome expectancies have also been shown to be predictive of prospective alcohol use (e.g. Goldman \& Darkes, 2004; Stacy, 1997; Stacy, Newcomb \& Bentler, 1991), however, it must be noted that the proportion of explained variance is much smaller than in studies that examined concurrent alcohol use (see Jones, Corbin, \& Fromme, 2001).

Although explicit alcohol expectancies have been proven successful in predicting alcohol use and abuse, their assessment has been criticized on a number of methodological and conceptual grounds (Leigh, 1989). Measuring explicit alcohol expectancies requires participants to rely on introspection; however, the question remains whether participants are willing and able to articulate the underlying motivational processes of their behavior (Stacy, 1997; Wiers et al., 2002; cf. Nisbett \& Wilson, 1977). Consequently, several authors proposed the use of indirect measures (Stacy, 1997; cf. Greenwald \& Banaji, 1995). These indirect measures do not rely on introspective awareness of ones reasons for engaging in certain behavior and are supposed to tap into more automatic underlying motivational processes (Greenwald \& Banaji, 1995; Greenwald, McGhee \& Schwartz, 1998). 


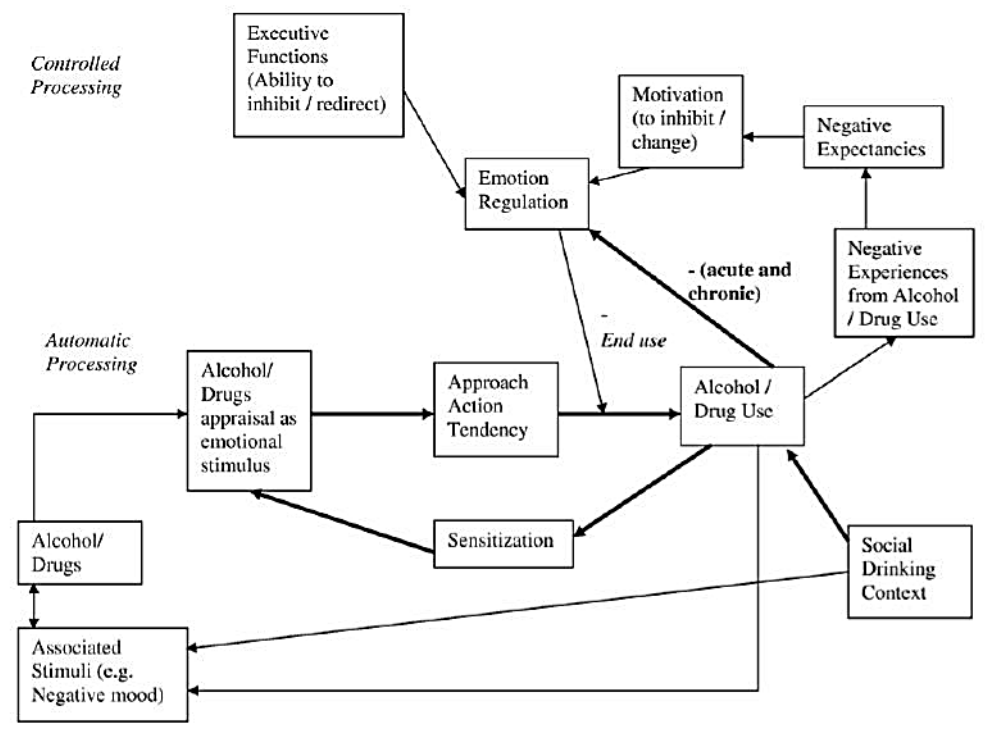

Figure 2. Dual process model on adolescent drinking by Wiers et al (2007).

\section{Implicit Alcohol-Related Cognition}

Recently, there has been an increased interest into the role of implicit cognitive processes in the development of addictive behaviors. Implicit cognitive processes represent relatively automatic associative motivational processes (Greenwald \& Banaji, 1995; Kahneman, 2003). Early experiences with alcohol could result in alcohol-related cues (e.g. presence of alcohol), outcomes (e.g. excitement), and behaviors (e.g. drinking) to become associated in memory. These associations may become strengthened over time and guide behavior relatively automatically (Stacy, 1997). For instance, when one is present at a party where alcohol is available, alcohol-related cues can automatically activate salient appetitive alcohol associations (such as the urge to feel excited) and consequently guide drinking behavior and enhance binge drinking in the current situation. Implicit associations with alcohol have been shown to predict unique variance in current and prospective alcohol use after controlling for explicit alcohol expectancies (e.g. Jajodia \& Earleywine, 2003; Palfai \& Wood, 2001; Stacy, 1997; Wiers et al., 2002).

Implicit cognition can be measured with various tasks derived from different research paradigms. For example, indirect word association tasks have been derived from basic memory research and found to predict substance use among college students (e.g. Kelly, Masterman, \& Marlatt, 2005; Palfai \& Wood, 2001; Stacy, 1995), community samples (e.g. Stacy \& Newcomb, 1998), drug offenders (e.g. Ames, Zogg, \& Stacy, 2002), and at-risk youth (e.g. Ames, Sussman, Dent, \& Stacy, 2005). With various 
indirect tests of word associations, it was shown that individuals who generated more drug-related responses to ambiguous drug-related cues were more likely to report higher levels of drug use than individuals who generated fewer drug-related responses. Word associations related to alcohol and other drugs are thought to reflect the likelihood that a given behavior (e.g. alcohol use) will be spontaneously triggered by relevant cues in a variety of settings (Stacy, 1997).

In addition, various reaction time paradigms have been adapted to assess implicit associations with alcohol. Among the most commonly researched are variants of the Implicit Association Test (IAT; Greenwald, McGhee, \& Schwartz, 1998) and the Extrinsic Affective Simon Task (EAST; De Houwer, 2003). The IAT is a response latency categorization task that evaluates the relative strength of contrasted associations (see Greenwald et al., 1998). In general, the IAT has been found to generate robust findings and acceptable reliabilities and to compare the association between contrast categories (e.g. Greenwald \& Nosek, 2001). However, this task has also been found to be sensitive to contextual variations (e.g. Mitchell, Nosek, \& Banaji, 2003) and has been criticized on its relative nature and the possibility that people employ recoding strategies (De Houwer, 2002; Rothermund \& Wentura, 2004).

Since the development of the IAT, other related reaction time measures, such as the Extrinsic Affective Simon Task (EAST, De Houwer, 2003), have been developed with their own strength and weaknesses. The EAST differs from the IAT in several ways. For instance, the EAST allows for the evaluation of a single association as well as several associations within the same task. Additionally, in the EAST associations are not contrasted with each other and as such the task measures associations more absolute. Both the EAST and IAT have been found to predict alcohol use and alcohol-related problems among college students (e.g. IAT: Jajodia \& Earleywine, 2003; Wiers et al, 2002; EAST: De Houwer, Crombez, Koster, \& De Beul, 2004; De Jong, Wiers, van den Braak, \& Huijding, 2007). For instance, with the use of the IAT, Wiers et al. (2002) found that heavy drinkers associated alcohol more strongly with arousal than with sedation, whereas light drinkers did not, and in addition these arousal associations predicted prospective alcohol use.

\section{Executive Functioning}

From a dual process perspective on addiction, executive functioning can be regarded as imperative in moderating the influence of implicit processes (Stacy et al., 2004; Wiers et al., 2007). Executive functions can be described as a set of cognitive skills relevant to goal-directed behavior involving different abilities such as shifting, updating and inhibition (Miyake et al., 2000). Working memory capacity has been proposed to be a central construct that possibly binds these different but related executive functions (Kane \& Engle, 2002). The relationship between executive functions and alcohol use has been shown to be bidirectional. Poorer executive functioning can be considered a risk 
factor for developing addictive behaviors such as drinking alcohol (e.g. Finn \& Hall, 2004; Peterson et al., 1992; Tapert, Baratta, Abrantes, \& Brown, 2002). Additionally, alcohol abuse has been shown to have negative consequences for the maturation of brain regions (e.g., DeBellis et al., 2000), causes impaired neuropsychological functioning (e.g., Brown et al., 2000) and altered processing in executive functioning tasks (Tapert et al., 2004). Prior research has suggested that the influence of implicit automatic processes is indeed moderated by executive control (e.g. Feldman-Barrett et al., 2004; Payne, 2005).

Finn and Hall (2004) proposed two mechanisms that might be responsible for the moderating influence of executive functioning on the implicit processes-behavior relationship. First, low activating capacity of working memory makes it difficult to shift attention away from highly activated stimuli to stimuli that are less salient. Second, short term positive associations with behavior tend to be highly activated (salient), whereas the long term negative associations with behavior are usually weakly activated. Consequently, in high-risk situations such as being at a party where alcohol is available, an individual needs to be able to switch to less salient goals - such as the intention not to drink large amounts of alcohol or other information such as the negative consequences of alcohol (mis)use - and attend to this information while distracting salient information in the current high risk situation is automatically activated (e.g., the urge to feel intoxicated). This relationship between executive functioning and behavior suggests that individuals who are less able to actively manage less salient but adaptive goals when faced with distracting information are more likely to let their behavior be guided by distracting salient information that is triggered in the current situation (e.g. Stacy et al., 2004; Wiers et al, 2007).

\section{Motivation}

Even if someone has a high level of executive functioning, one still needs to be motivated or have a goal in mind to apply this executive control (Feldman-Barrett et al., 2004; Wiers et al, 2007). Thus, the concept of executive functioning does not replace the importance of motivation and goals, just as the concept of motivation does not replace the importance of the capacity to restrain one-self (Towles-Schwen \& Fazio, 2006). Indeed, prior research has shown that the influence of implicit racial stereotypes on social behavior increases when an individual has no motivation to control these automatic racial attitudes (Olson \& Fazio, 2004).

The importance of having motivation to control one's impulses has been demonstrated by a large body of research in the context of addictive behaviors (Milner \& Rollnick, 2002). Individuals with more adaptive motivational structures are better able to control their drinking than those with maladaptive motivational structures. Having more adaptive motivational structures can be defined as having positive goals in life that an individual wants to achieve, feeling optimistic about achieving these goals, and the 
satisfaction that will ensue. Research showed that in treatment alcohol abusers with adaptive motivational structures show a better response to treatment, less problem denial and more motivation for change than those with maladaptive motivational structures (Cox \& Klinger, 2002).

\section{ALCOHOL USE \& INTERVENTION}

In the Netherlands, primary prevention in the form of school based alcohol and drugs education is stimulated and facilitated by the Dutch government. An example of such a program is the 'Healthy School and Drugs' project, for which only some small beneficial effects on alcohol use were reported. Additionally, a paradoxical intervention effect on marijuana use was found (Cuijpers, Jonkers, De Weerdt, \& De Jong, 2002). Even though these results are not very positive, it is estimated that each year at least 350,000 secondary school students (ages 12 to 18) receive this intervention. While primary preventive interventions does sometimes show protective effects in terms of adolescent drinking (e.g. Botvin, Griffin, Diaz, \& Ifill-Williams, 2001; Botvin \& Griffin, 2004), reviews generally show low levels of effectiveness (Foxcroft, Ireland, Lister-Sharp, Lowe \& Breen, 2003). Therefore, there is an additional necessity to develop secondary or targeted preventive intervention for adolescents who are, despite all preventive efforts, involved in hazardous alcohol and/or drug use.

Although research has provided insight into consequences of adolescent drinking and risk factors for developing alcohol problems, the question remains what comprises an effective targeted intervention to modify drinking in those at-risk adolescents. For example, intervention research has mainly focused on the development of effective targeted interventions for (young) adults. In diverse adult populations, different forms of targeted interventions have been demonstrated to be modifying hazardous health behavior. For example, while in treatment-seeking populations extended interventions showed better outcomes than brief interventions, non-treatment seeking adults receiving a brief intervention effectively reduced alcohol consumption compared with assessment-only controls (Moyer, Finney, Swearingen, \& Vergun, 2002). Additionally, the clinical implication from a dual process perspective of addictive behavior would be that interventions can be effective in reducing hazardous drinking by changing (explicit or implicit) alcohol-related cognition, targeting executive functioning and adding a motivation enhancement aspect. And although research has implicated all these factors to be important in the development of addictive behaviors(e.g. Stacy et al., 2004; Wiers et al, 2007), there is still much unknown about how to use this knowledge in developing interventions for at-risk adolescents and what the precise underlying mechanism are through which these interventions might work.

For example, interventions that consist of brief Motivational Interviewing (Ml; Miller \& Rollnick, 2002) have consistently yielded greater support for their efficacy to reduce 
hazardous drinking in adults compared with educational or information-only interventions (e.g. Marlatt et al., 1998, see for review, Larimer \& Cronce, 2002). Motivational interventions might be one of the most promising approaches especially in difficult atrisk non-treatment seeking populations (Grenard et al., 2007; Moyer et al., 2002). However, there is still much unknown about the cognitive mechanisms underlying $\mathrm{Ml}$ effects. Modifying readiness to change is seen as an important precursor to behavior change (Miller \& Rollnick, 2002). Therefore, increasing readiness to change might be an important underlying mechanism through which MI works. Even so, only a few studies have tried to identify the cognitive mechanisms through which MI might work (e.g. Bosari \& Carey, 2000; Wood, Capone, Laforge, Erickson \& Brand, 2007) and there is as yet little evidence for it's effectiveness in (at-risk) adolescents, although it should be noted there is little research yet (for review see Grenard, Ames, Pentz \& Sussman, 2006).

\section{OUTLINE OF THIS THESIS}

As pointed out previously there are several reasons why there is an additional necessity to develop secondary or targeted preventive intervention for adolescents who are, despite all preventive efforts, involved in hazardous alcohol and/or drug use. Because of the current lack of effective targeted interventions in the Netherlands and questions regarding which targeted determinants of alcohol use will ultimately change subsequent drinking behavior, Chapter 2 describes a study in which the effectiveness of a targeted intervention program aimed at at-risk adolescents was investigated in a randomized clinical trial. This program combined intervention methods which have been proven effective in reducing drinking in young adults, such as an expectancy challenge, cognitive behavioral skill training and brief motivational feedback. Additionally, this intervention contained the new element of discussing biological, cognitive and social risk factors for developing alcohol problems. Thus, by focusing on changing more explicit alcohol-related processes, for instance by reducing positive alcohol expectancies for a high dose of alcohol, the aim was to moderate adolescent alcohol use.

However, besides explicit cognitive processes, dual process models of addiction state that also more implicit appetitive cognitive processes might play an important role in the development of addictive behaviors (Stacy et al., 2004; Wiers et al, 2007). Consequently, in Chapter 3 it was investigated whether these implicit appetitive alcohol-related cognitions predicted adolescent binge drinking after one year above and beyond several other variables, such as explicit alcohol-related cognitions. Additionally, it was investigated whether younger and older adolescents differed in their alcoholrelated cognition using a cross-sectional design. It was expected that with increasing age and drinking experience, adolescents had stronger explicit and implicit appetitive 
alcohol-related cognitions and weaker explicit and implicit negative alcohol-related cognition.

These implicit alcohol-related cognitions can be measured with various indirect tasks derived from different research paradigms. However, given the wide range of different indirect measures and the fact that assessment procedures are not always process pure (e.g. Houben \& Wiers, 2006), it is necessary to evaluate the predictive value of these indirect assessment procedures next to other more direct assessment procedures. Chapter 4 deals with the question how three of these indirect measures (IAT, EAST and word association tasks) performed as indirect measures of alcoholrelated cognition in the prediction of alcohol use in at-risk adolescents.

In addition, besides questions regarding the assessment procedure, the predictive value of these implicit appetitive cognitive processes might also be influenced by explicit reflective cognitive processes. In turn, these explicit reflective cognitive processes are thought to be moderated by motivation and executive functioning (e.g. Stacy et al, 2004; Wiers et al 2007). Therefore, Chapter 5 describes a study in which both the interactions between implicit alcohol-related cognitions and working memory capacity and explicit alcohol-related cognitions and working memory capacity were explored in the prediction of alcohol use in at risk adolescents. It was hypothesized that the influence of implicit appetitive associations on subsequent drinking behavior is stronger in adolescents with a below average level of working memory capacity than in adolescents with an above average level of working memory capacity. Conversely, the influence of explicit positive-arousal alcohol-related processes on subsequent drinking behavior is hypothesized to be stronger in adolescents with an above average level of working memory capacity compared with adolescents with a below average level of working memory capacity. Additionally, in Chapter 6 a study is evaluated to determine the influence of Motivational Interviewing (Miller \& Rollnick, 2002) on implicit and explicit alcohol-related cognitions and subsequent alcohol use in at-risk adolescents. From a dual process perspective, it was expected that by enhancing motivation to change, negative alcohol expectancies would moderate the influence of implicit positive-arousal associations on drinking behavior.

Finally, Chapter 7 provides a general overview of the main findings of this thesis. These main findings will be discussed from a dual process perspective and will be related to research on implicit and explicit cognitive processes, intervention and adolescent development. Finally, the implications for future experimental and clinical research on adolescent alcohol use are discussed. 
GENERAL INTRODUCTION 



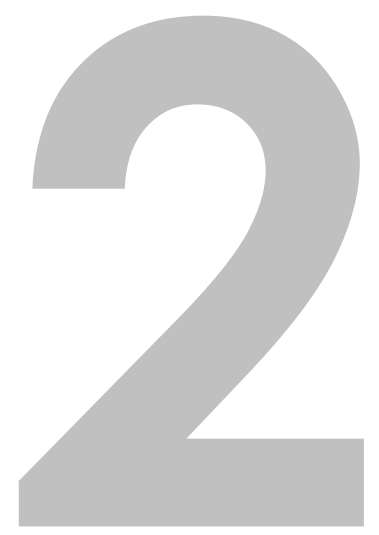

A randomized clinical trial of a targeted intervention to moderate alcohol use and alcohol-related problems in at-risk adolescents

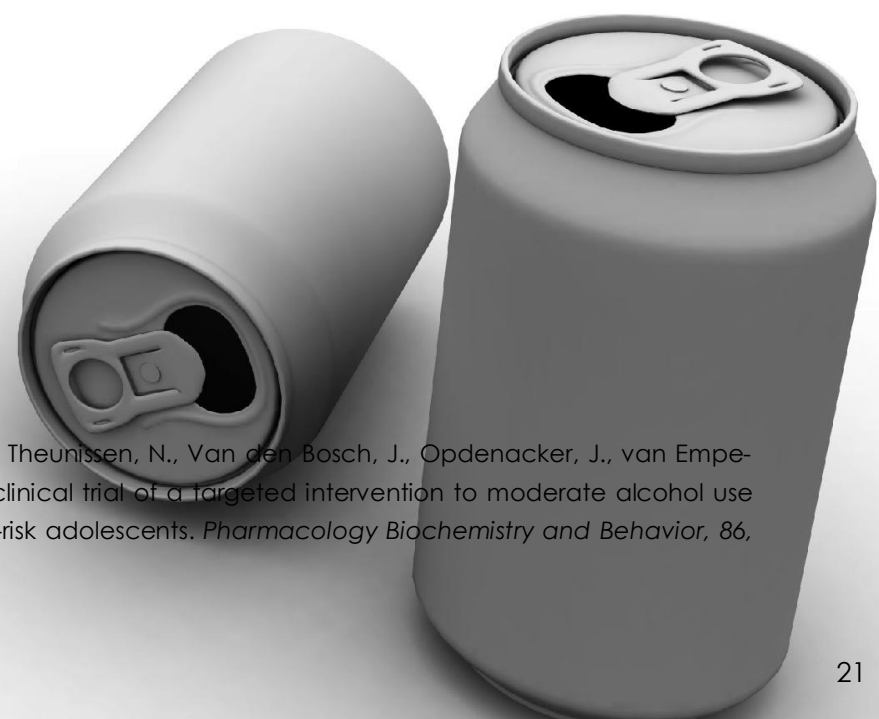




\section{ABSTRACT}

This study investigated the effectiveness of a targeted intervention program aimed at at-risk adolescents in a randomized clinical trial design ( $N=107)$. This program combined intervention methods which have been proven effective in reducing drinking in young adults, such as an expectancy challenge, cognitive behavioral skill training and brief motivational feedback. Additionally, this intervention contained the new element of discussing biological, cognitive and social risk factors for developing alcohol problems. We investigated whether this seven session program was successful in changing cognitive determinants of drinking behavior and consequently in moderating alcohol use and the development of alcohol-related problems in at-risk adolescents. The intervention was effective in changing several of the targeted cognitive determinants. However, despite the changes in these cognitive determinants of drinking, the experimental group did not show a significant difference in decrease of drinking at post-test compared with the control group. The results did not yield support for any differential long term effects of the intervention. We concluded that although the present intervention successfully changed important cognitive determinants of drinking more is needed to change subsequent drinking behavior in at-risk adolescents. 
Adolescent alcohol abuse is a serious problem. Research has shown that alcohol abuse among adolescents increases the probability of severe short term consequences like forced and unsafe sex, dropping out of school, unemployment, social isolation, and depression (e.g. Newcomb \& Bentler, 1989). Furthermore, alcohol abuse during adolescence has negative long term consequences. Research has indicated that an early onset of drinking during adolescence predicts alcohol-related problems later in life (Grant \& Dawson, 1997). Additionally, animal research has demonstrated that the long term effects of binge drinking on the adolescent brain differ and are more severe compared with the effects on the adult brain (e.g. Crews, Braun, Hoplight, Switzer, \& Knapp 2000; White et al., 2000; White \& Swartzwelder, 2004). Moreover, studies involving human adolescents have revealed that alcohol abuse has negative consequences for the maturation of brain regions (e.g. DeBellis et al., 2000), causes impaired neuropsychological functioning (e.g., Brown, Tapert, Granholm, \& Dellis, 2000) and altered processing in executive functioning tasks (Tapert et al., 2004). All these reasons suggest that adolescence is a very important period to try to moderate hazardous adolescent drinking to safer levels.

Although primary preventive interventions shows protective effects in terms of adolescent drinking (e.g. Botvin, Griffin, Diaz, \& Ifill-Williams, 2001; Botvin \& Griffin, 2004), there is an additional necessity to develop secondary preventive intervention for adolescents who are, despite all preventive efforts, involved in hazardous alcohol and/or drug use. Although research has provided insight into consequences of adolescent drinking and risk factors for developing alcohol problems, the question remains what comprises an effective targeted intervention to modify drinking in those at-risk adolescents. Until now, research has mainly focused on the development of effective targeted interventions for (young) adults. In diverse adult populations, different forms of targeted interventions have been demonstrated to be modifying adult hazardous health behavior. For example, while in treatment-seeking populations extended interventions showed better outcomes than brief interventions, non-treatment seeking adults receiving a brief intervention effectively reduced alcohol consumption compared with assessment-only controls (Moyer, Finney, Swearingen, \& Vergun, 2002). Since some of those at-risk adolescents could be classified as being somewhere in between non-treatment seeking (e.g. not yet ready to change their hazardous behavior) and treatment-seeking (e.g. being aware of having problems in school and/or at home because of drinking), it might be most effective to use a moderately long targeted intervention program to reduce hazardous drinking. Moreover, cognitive behavioral skills-based interventions and brief motivational feedback have consistently yielded greater support for their efficacy to reduce hazardous drinking in adults compared with educational or information-only interventions (Larimer \& Cronce, 2002). Intervention programs which combine social learning and cognitive behavioral principles, such as the Alcohol Skills Training Program (ASTP; Fromme, Marlatt, Baer, \& Kivlahan, 1994) and the related Brief Alcohol Screening and Intervention for College Students (BASICS; 
Dimeff, Bear, Kivlahan, \& Marlatt, 1999), show promising effects of moderating drinking in young adult drinkers. Since at-risk adolescents presumably have similar problems compared with young adults, it might perhaps be most effective to combine cognitivebehavioral skills training with motivational feedback within one intervention program. Furthermore, alcohol outcome expectancies have been shown to have a large influence on subsequent alcohol use (Goldman, Del Boca \& Darkes, 1999). Challenging these hazardous alcohol outcome expectancies is an effective method in decreasing drinking in young adults (Darkes \& Goldman, 1993; 1998; Wiers, Van de Luitgaarden, Van den Wildenberg, \& Smulders, 2005). Consequently, changing hazardous alcohol outcome expectancies in at-risk adolescents might positively modify the influence of these risk factors on the subsequent drinking behavior.

Moreover, Wiers, Gunning and Sergeant (1998) proposed to use another element in targeted interventions to moderate drinking in at-risk adolescents, namely raising awareness for common misconceptions concerning risk factors for developing alcohol problems. Perceived vulnerability or someone's subjective perception of his personal risk is an important factor in predicting behavioral change (Weinstein, 1982). Drinkers are prone to misinterpret risk factors, such as the influence of family history of drinking, level of response to alcohol, hazardous alcohol outcome expectancies, susceptibility to peer pressure, gender, age of onset, other drug use and externalizing and internalizing problem behavior (Wiers et al., 1998). Although Schuckit (1998) actually proved the opposite to be true, a common misconception is that someone who experiences few negative sedative effect of drinking will have smaller chance of developing alcohol problem. In general, these misconceptions have been shown to be very resilient and to exist in several knowledge domains such as physics, psychology and biology (Wellman \& Gelman, 1992). One of the most effective ways to challenge people's current misconceptions is to enhance deep processing strategies by discussing anomalous data and presenting a plausible alternative theory (Chinn \& Brewer, 1993). Consequently, educating drinkers in an interactive way about the biological, cognitive and social risk factors for developing alcohol-related problems next to effectively training them in cognitive behavioral skills and enhancing motivation for change may be an effective way to moderate their hazardous drinking.

The current study investigated the effectiveness of the targeted intervention program "Learning to Drink" in a randomized clinical trial design. The aim was to investigate whether this program was effective in changing cognitive determinants of drinking and thus in moderating alcohol use and the development of alcohol-related problems in at-risk adolescents. The program combined intervention methods such as brief motivational feedback and cognitive behavioral skills-based interventions (Larimer \& Cronce, 2002) and was partly based on the Alcohol Skills Training Program (ASTP; Fromme et al., 1994) and the related Brief Alcohol Screening and Intervention for College Students (BASICS; Dimeff et al., 1999). It was hypothesized that the targeted cognitive determinants of drinking behavior would effectively change in the experi- 
mental group compared with the information-only control group. The perception of risk factors was expected to increase, whereas the positive alcohol expectancies for a large dose of alcohol were expected to decrease as a function of the intervention. Consequently, we hypothesized that this change in cognitive determinants would lead to a moderation of the increase in alcohol use and alcohol-related problems in the experimental group compared with the information-only control group. Finally, we explored if the intervention would be effective over a longer period in moderating alcohol use as well.

\section{METHOD}

\section{Recruitment}

High school students of different educational levels were approached to participate in this study. Several different recruitment strategies, such as recruitment through written advertisements, school nurses and youth workers, were used and proved to be largely unsuccessful. More than 7000 adolescents were approached out of which only 22 adolescents were included (the negative response was primarily due to lack of reaction to the advertisement). Direct contact in schools through classroom recruitment talks proved to be a better strategy in recruiting participants. The aim of these talks was that regular drinking adolescents (drinking alcohol at least once every two weeks) felt impelled to participate in 'Learning to Drink', advertised as a fun program in which adolescents learned to drink alcohol in a healthy way (with the metaphor of learning how to drive a car). Practical information was given on how many sessions the adolescents were to attend, where and when the sessions took place and the monetary incentives that were given. Furthermore, information sheets for the adolescents and their parents were handed out. Out of approximately 2000 distributed information sheets 102 adolescents replied out of which 85 could be included in the program.

\section{Participants}

A total of 107 participants ( 61 male, 46 female) were included at pre-test and randomly divided over the experimental intervention group $(n=53)$ and information-only control group ( $n=54$ ). The experimental intervention group and the information-only control group were subsequently divided in small groups of approximately 10 participants. The age of the participants ranged from 14 to $18(M=15.51, S D=1.01)$. On average the participants consumed 9.11 Dutch standard alcoholic drinks per week ( $S D=7.85$ ) (a standard alcohol serving in the Netherlands contains somewhat less alcohol than an English or American standard alcohol serving: 12 vs. 14 gr.). Out of the 107 participants $77(71.9 \%)$ indicated having one or more binge episodes in the last two weeks. 
Table 1

Pearson Correlation and Cronbach Alphas for Perception of Risk Factors, Alcohol Expectancies, Alcohol Use and AlcoholRelated Problems at Pre-test

\begin{tabular}{|c|c|c|c|c|c|c|c|c|c|c|c|c|}
\hline & \# Items & $\alpha$ & 1 & 2 & 3 & 4 & 5 & 6 & 7 & 8 & 9 & 10 \\
\hline 1. Perc. Risk Factors & 13 & .67 & - & & & & & & & & & \\
\hline 2. Pos. Alc. Exp. LD & 16 & .88 & .02 & - & & & & & & & & \\
\hline 3. Pos. Alc. Exp. HD & 11 & .86 & .06 & $.76^{* *}$ & - & & & & & & & \\
\hline 4. Neg. Alc. Exp. LD & 11 & .86 & $.25^{*}$ & $.40^{* *}$ & $.38^{* *}$ & - & & & & & & \\
\hline 5. Neg. Alc. Exp. HD & 10 & .74 & $.26^{*}$ & .16 & $.29^{* *}$ & $.62^{* *}$ & - & & & & & \\
\hline 6. Alc. Week & 7 & .49 & -.05 & $.35^{* *}$ & $.44^{* *}$ & .15 & .06 & - & & & & \\
\hline 7. Alc. Week Day & 4 & .30 & .04 & .03 & .08 & .07 & .09 & $.54^{* *}$ & - & & & \\
\hline 8. Alc. Weekend Day & 3 & .50 & -.06 & $.38^{* *}$ & $.47^{* *}$ & .15 & .05 & $.99 * *$ & $.39 * *$ & - & & \\
\hline 9. Alc. Binge & 7 & .38 & -.05 & $.33^{* *}$ & $.42^{* *}$ & .14 & .05 & $.85^{* *}$ & $.35^{* *}$ & $.86^{* *}$ & - & \\
\hline 10. RAPI & 17 & .74 & -.01 & $.38^{* *}$ & $.52^{* *}$ & .18 & $.30 * *$ & $.37^{* *}$ & .11 & $.39 * *$ & $.35^{* *}$ & - \\
\hline
\end{tabular}

Note. Perception of risk factors scores were average scores on an unmarked $100 \mathrm{~mm}$ Visual Analogue Scales; all alcohol expectancy scores were average scores on a 5-point Likert scale ranging from strongly disagree to strongly agree; all RAPI scores were average scores on a 5-point Likert scale ranging from never to daily. Perc. Risk Factors = Perception of Risk Factors; Pos. Alc. Exp. LD = Positive Alcohol Expectancies for Low Dose; Pos. Alc. Exp. HD = Positive Alcohol Expectancies for High Dose; Neg. Alc. Exp. LD = Negative Alcohol Expectancies for Low Dose; Neg. Alc. Exp. HD = Negative Alcohol Expectancies for High Dose; Alc. Week = combined number of standard drinks for each day of the week; Alc. Week Day = mean number of standard drinks on a week day; Alc. Weekend Day = mean number of standard drinks on a weekend day; Alc. Binge $=$ combined number of binges per week; RAPI $=$ mean index of alcohol-related problems. ${ }^{*} p<.05$, two-tailed. ${ }^{* *} p<$ .01 , two-tailed.

\section{Material}

\section{Alcohol Use}

Alcohol use was measured with a shortened version of a Dutch self-report alcohol use questionnaire (Wiers, Hoogeveen, Sergeant \& Gunning, 1997), based on the timeline follow-back method (Sobell \& Sobell, 1990). Self-report questionnaires have been proven to be reliable and valid if the soberness of the participant and confidentiality of data is assured (Sobell \& Sobell, 1990). Both requirements were fulfilled in this study. At pre-test, post-test, six month follow-up and one year follow-up, participants indicated how many standard alcoholic drinks they consumed for each day of the past week. From this alcohol consumption measure the number of standard alcoholic drinks per week, the mean number of standard alcoholic drinks on a weekday (Monday to Thursday), the mean number of alcoholic drinks on a weekend day (Friday to Sunday) and the number of binges (five standard alcoholic drinks or more per day) per week were calculated. Each alcohol consumption measure had a moderate internal consistency with Cronbach's alphas ranging from .30 to .50 (see Table 1).

\section{Alcohol-Related Problems}

An index of alcohol-related problems was assessed using an adapted version of the Rutgers Alcohol Problems Index (White \& Labouvie, 1989). Previously, it has been shown that a shortened version of the RAPI correlated .99 with the original 23-items version 
(White \& Labouvie, 2000). We used a shortened version of the RAPI which consisted of 17 items related to personal consequences of alcohol use. Participants were asked to indicate on a 5-point Likert scale (ranging from never to daily) how many times they experienced certain problems within the last six months because of their alcohol use. Examples of items are: "Caused shame or embarrassment to someone" and "Not able to do your homework or study for a test. The items were summed to create an index of alcohol-related problems (Cronbach's alpha $=.74$ ).

\section{Alcohol Outcome Expectancy}

Participants were asked to fill out a 48 items questionnaire briefly describing situations (e.g. "After studying hard, I relax from drinking a few glasses of alcohol") that assessed positive and negative expectancies for a low and for a high dose of alcohol (Wiers et al., 1997). Participants had to indicate to what extent they (dis)agreed with each item on a 5-point Likert scale (ranging from strongly disagree to strongly agree). An average alcohol expectancy score was calculated for each of the subscales. Each subscale had a good internal consistency with Cronbach's alphas all above .70 (see Table 1)

\section{Perception of Risk Factors}

Participants were asked to fill out a 14 items questionnaire with statements briefly describing risk factors, such as "somebody who mainly experiences the positive effects of drinking alcohol will have a higher risk of becoming alcohol dependent than somebody who experiences these positive effects to a lesser extent". Participants had to indicate to what extent they (dis)agreed with each item on a $100 \mathrm{~mm}$ unmarked Visual Analogue Scale (VAS; ranging from strongly disagree to strongly agree). An average VAS score was calculated as an index for the perception of risk factors (Cronbach's alpha $=.67)$.

\section{Procedure}

After this study was approved by an institutional medical ethical committee, participants were recruited. When active consent was obtained from both the participants and parents, participants were included in the project.

Pre-test

The 107 included participants and their parents were invited in small groups (of approximately 10 people) to the bar-lab at Maastricht University. After the parents were accompanied to another room and the confidential and anonymous nature of the data was emphasized, the participants were asked to fill out the alcohol use questionnaire, the questionnaire on alcohol-related problems (RAPI), the alcohol expectancy questionnaire and additional questionnaires on background variables needed for the feedback given in the individual seventh session. Instructions were given on how to fill 
out the questionnaires and the experimenter was present to answer participants' questions. Meanwhile, the parents in the other room were also asked to fill out several questionnaires (e.g. regarding family history, their own drinking and communication with their son or daughter)

\section{Intervention}

The program "Learning to Drink" consisted of a total of seven weekly sessions and of one parent session which took place in a bar lab at the Maastricht University. The seven weekly sessions for the adolescents consisted of six 90 minutes group-sessions and one final individual motivational interview. The intervention sessions were carried out by four counselors who were all trained in Motivational Interviewing and in using the protocol for this intervention. All the trainers were either master students or graduate students in Psychology.

PARENT SESSION. During the parent session a couple of topics were addressed. First, some background information was given about alcohol use among adolescents. Furthermore, suitable adolescents drinking norms for moderate drinking were given and discussed. Subsequently, it was specified how parents could detect signals of heavy alcohol use and alcohol-related problems and how parents could communicate with their child regarding his or her alcohol use. Several options of how parents could respond to their child's alcohol use were discussed in an interactive way.

SESSION 1. The first session for the adolescent participants contained the introduction to the program. During this session again the definition of standard alcoholic drink was explained. Participants were told how to fill out alcohol diaries which they had to keep during the seven week intervention period.

SESSION 2. The second session consisted of a social expectancy challenge (Darkes \& Goldman, 1993) which was adapted for mixed-gender group (Wiers \& Kummeling, 2004). During this session participants were made to believe they all received an alcoholic drink while actually half of them received a glass containing a placebo. This procedure was adapted for adolescents: participants received one alcoholic drink or placebo instead of two. After the true nature of this procedure was revealed, the informational part of the session focused on social alcohol expectancies vs. the real effects of alcohol. Further, alcohol expectancies in commercials were discussed in an interactive way. Before leaving, participants blood-alcohol levels were measured with a breathalyzer, and participants signed for the level measured and were instructed to be extra careful given the presence of some alcohol in their blood. After the experiment, no participant was above the legal limit ( 0.5 per mil).

SESSION 3. The third session consisted of a sexual expectancy challenge (Darkes \& Goldman, 1993) adapted for mixed-gender group (Wiers \& Kummeling, 2004) and for adolescents (participants received one alcoholic drink or placebo instead of two). The informational part of the session focused on sexual expectancies vs. real alcohol effects followed by explanation of the blood alcohol curve and gender differences in the 
effects of alcohol and in the risks associated with heavy alcohol use (based on BASICS, Dimeff et al., 1999). Again, participants blood-alcohol levels were measured with a breathalyzer and participants signed for the level measured and were instructed to be extra careful given the presence of some alcohol in their blood. After the experiment, no participant was above the legal limit ( 0.5 per mil).

SESSION 4. In the fourth session drinking norms (based on ASTP approach, Fromme et al., 1994) and decisional balance of light and heavy drinking (Miller \& Rollnick, 2002) were discussed. Participants were asked to estimate if they drank more, less or equal amounts of alcohol compared with their peers. After making their estimation, each participant received personalized normative feedback. At group-level it was discussed what the causes were for discrepancies between the estimation of ones own alcohol use compared with others and the actual normative comparison. Finally, the participants were asked what they considered to be the advantages and disadvantages of moderate and heavy alcohol use. The most common advantages and disadvantages of moderate and heavy alcohol use were discussed within the group.

SESSION 5. The fifth session dealt with the perception of risk factors for developing an alcohol problem. A new element in the intervention context was the use of a "naive theory" approach (cf. Chinn \& Brewer, 1993): common misconceptions regarding risk factors for developing an alcohol problem were first elicited, then discussed and targeted. Information was given about risk factors such as family history, alcohol outcome-expectancies, externalizing or internalizing personality characteristics and other drug-use.

SESSION 6. The sixth session consisted of refusal efficacies skills training (based on the ASTP approach, Fromme et al., 1994). Participant were first asked to identify high risk situations in which they usually drink more than planned and write a short script about an actual event that took place in one of these high risk situations. The participants practiced in saying no to alcoholic drinks by role playing one of the high risk situation scripts. Alternatives refusal strategies and alternative behaviors to avoid binge drinking were discussed.

SESSION 7. The seventh session was an individual session in the form of an adapted motivational interview in which individualized feedback on the adolescent drinking behavior and risk factors was provided (based on the BASICS program, Dimeff et al., 1999). During this individual session various counseling skills were used (such as reflective listening, affirmations, open ended questions and summaries) in order to elicit 'change talk' (Miller \& Rollnick, 2002). This created an opportunity for the participants to think and talk about their own alcohol use (also in comparison to their peers), risk factors and high risk situations, the advantages and negative consequences of drinking alcohol. The main objective was to stimulate new thinking on their personal alcohol use which might result in behavioral change. 
Information-Only Control Group.

After filling out the pre-test, participants in the control group received an information sheet about the biological and cognitive effects of alcohol, risk factors for developing an alcohol problem and the negative consequences of heavy drinking. During this seven week period the participants in the control group, as the participants in the experimental group, filled out several self-report questionnaires. They were individually contacted through mail. In an accompanying letter the confidential and anonymous nature of the data were emphasized and the participants were asked to fill out the enclosed questionnaires. Participants were requested to return the filled out questionnaires in a prepaid envelope.

Post-test

Directly after the intervention was finished, the 107 included participants and their parents were individually contacted by mail. In an accompanying letter the confidential and anonymous nature of the data was emphasized and the participants were asked to fill out the enclosed questionnaires (alcohol use questionnaire and RAPI) and return them in a prepaid envelope.

Follow-up after six months and one year

Approximately six month and one year after the beginning of the intervention, participants were again contacted by mail. In an accompanying letter the confidential and anonymous nature of the data was emphasized and the participants were asked to fill out the enclosed in questionnaires (alcohol use questionnaire and RAPI) and to return them in a prepaid envelope.

\section{Data reduction and statistical analysis}

There were several reasons to decide to calculate a drink index score. First, the five alcohol use outcome variables were significantly but only partially correlated (see Table 1). Therefore, they reflect different aspects of adolescents drinking. In order to be able to consider all these different aspects of adolescent drinking at once, we decided to calculate a mean drink index. Secondly, by calculating and log transforming the mean drink index we were able to obtain a normally distributed outcome measure out of five extremely positively skewed outcome measures. Finally, by calculating one mean drink index it was possible to analyze the effectiveness of the intervention with one instead of five different alcohol use outcome measures. This reduced the chances of making a Type I error. We computed the drink index score in three consecutive steps. First, z scores were calculated for five different outcome measures, namely the number of standard alcoholic drinks per week, the mean number of standard alcoholic drinks on a 


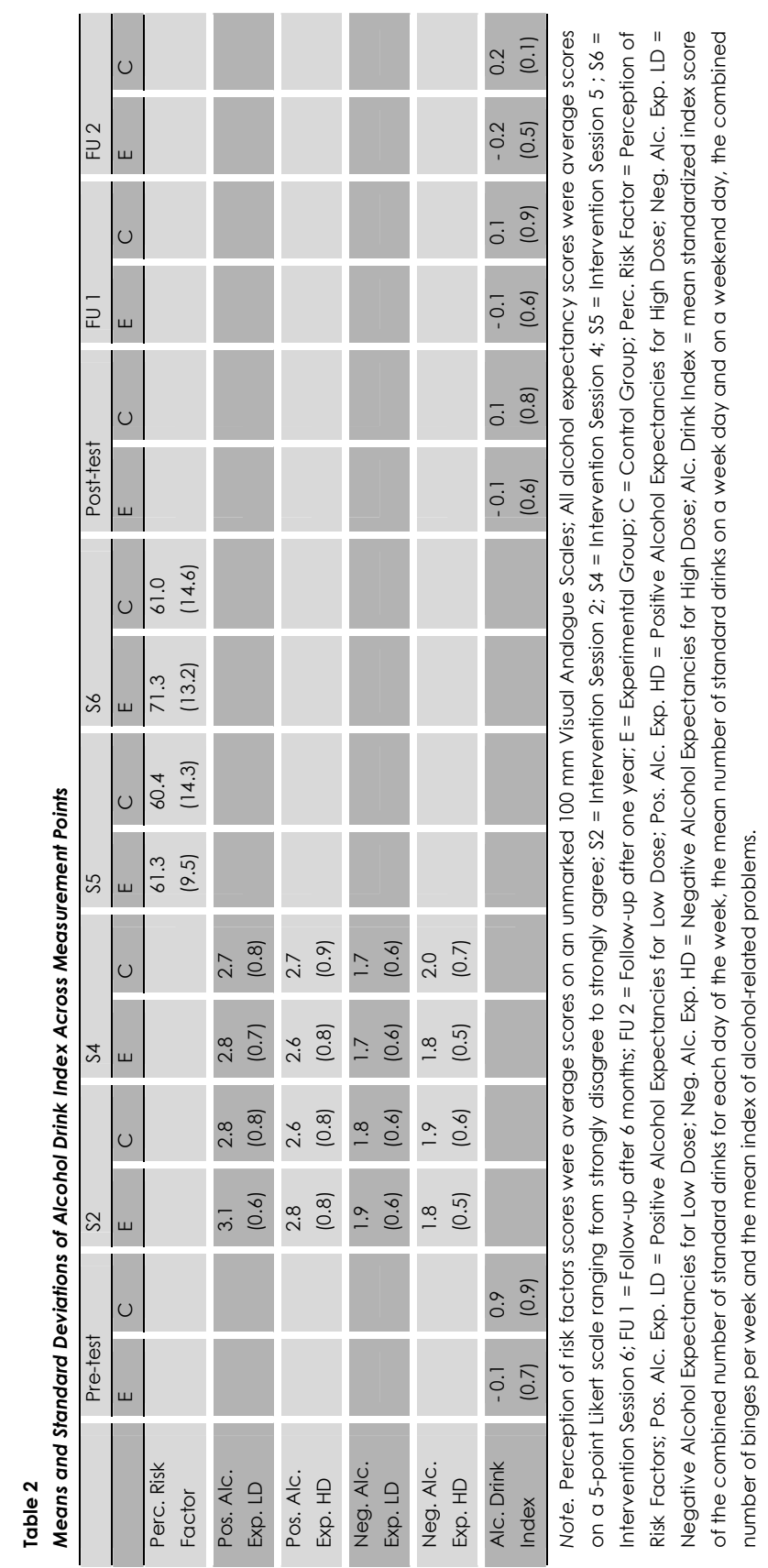


weekday, the mean number of standard alcoholic drinks on a weekend day, the number of binges per week and the total sum score on the RAPI. Subsequently the drink index score was computed by calculating the mean of these five z-scores. Finally, the drink index was log transformed to obtain a normally distributed dependent variable. From now on the log transformed drink index will be referred to throughout the text as the drink index.

All short term effects of the intervention on the targeted cognitive determinants were analyzed around the intervention sessions where the expected changes were to take place. The short term effects of the intervention on the drink index were analyzed from pre-test to post-test. The long term effects of the intervention on drink index were analyzed along pre-test, post-test, six month follow-up and one year follow-up.

\section{RESULTS}

\section{Drop out rates}

A total of 90 out of 107 participants $(84 \%)$ stayed in the program and completed the post-test assessments. Out of the 17 participants who dropped out 6 participants belonged to the control group and 11 participants belonged to the experimental group $\left(\chi^{2}(1)=1.86, p=.17\right)$. This indicates that there was no strong evidence for selective drop out. After six months, 82 of the 107 participants $(77 \%)$ returned the filled out follow-up questionnaires. After one year, 83 of the 107 participants $(78 \%)$ returned the filled out follow-up questionnaires.

\section{Short term effects of the Intervention}

Perception of Risk Factor

A 2(Time) X 2(Condition) Mixed ANCOVA controlling for age and gender revealed a significant Time $\times$ Condition effect for the perception of risk factors, $F(1,92)=19.85, p<$ .001 . The experimental group showed a significant increase in perceived risk factors between the fifth and the sixth session, $t(47)=5.99, p<.001$, whereas the control group did not, $t(47)=0.93, p>.50$.

\section{Alcohol Outcome Expectancies}

A 2(Time) X 2(Condition) Mixed ANCOVA controlling for age and gender revealed a significant Time $\times$ Condition effect for high dose positive alcohol expectancies, $F(1,91)$ $=5.72, p<.05$. Between the second and fourth session, a significant decrease in high dose positive expectancies was found in the experimental group, $t(49)=-3.35, p<.01$. There was no evidence for a significant change in high dose positive expectancies in the control group, $\dagger(44)=0.40, p>.50$. 
Additionally, a borderline significant Time $\times$ Condition effect for low dose positive alcohol expectancies was found, $F(1,91)=3.34, p=.07$. The experimental group showed a significant decrease in low dose positive expectancies between the second and fourth session, $t(49)=-3.97, p<.001$, the control group did not, $t(44)=-1.07, p=$ .29 .

Finally, there was a significant Time effect for low dose negative alcohol expectancies, $F(1,91)=13.12, p<.001$. Both controls and experimental group decrease their negative alcohol expectancies for a low dose of alcohol between the second and fourth session (see Table 2).

\section{Drink Index}

A 2(Time) X 2(Condition) Mixed ANCOVA controlling for age and gender did not show a significant Time $x$ Condition effect for drink index, $F(1,86)=1.08, p=.30$. The experimental group showed no significant decrease on the drink index compared with the control group (see Table 2).

\section{Long Term effect of the Intervention}

We analyzed the long term effects of the intervention by constructing a Latent Growth Model (LGM; Meredith \& Tisak, 1990) using AMOS 4.0 (Arbuckle \& Wothke, 1999). LGM methods are a form of Structural Equation Modeling (SEM) and offer several advantages over traditional methods for assessing change over time. First, because LGM methods are able to model both inter- and intra-individual variability in change, they can give a more accurate estimation of the differential effect of condition in change over time. Second, because LGM methods can include several measurement points in outcome assessment, they offer maximized information on the individual change over time compared with simple pre- and post-test designs. An additional advantage is that LGM methods can easily handle missing data which might especially useful in the context of analyzing data of randomized clinical trials (see Hess, 2000).

Based on the delta $\chi^{2}$ test, we decided that the model represented in Figure 1 fitted the data relatively well compared with more elaborate models, such as a model with a quadratic effect of time. This model was accepted as fitting the data $\left(\chi^{2}(7)=\right.$ 9.36, $p=.28$, RMSEA $=.056, \mathrm{CFI}=.984)$. Note that in SEM a non-significant $p$-value denotes a non-significant misfit of the model to the data and a model is regarded as fitting the data reasonably well when RMSEA $<.08$ and CFI >.90. As indicated in Figure 1, the latent factors intercept and slope are extracted from the drink index data across pre-test, post-test, 6 month follow-up and 1 year follow-up (i.e. from the drink index at 0 , 7, 26 and 52 weeks). These are used as estimates of baseline functioning (intercept) and the increase or decrease of the drink index over time (slope). The double-headed arrow between intercept and slope indicates the association between the individual level of baseline functioning and the individual change over time. By fixing all paths 


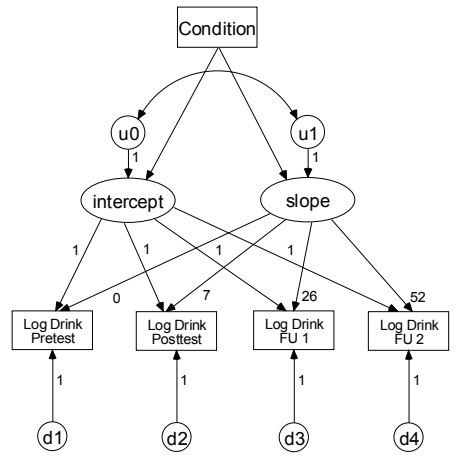

Figure 1. Latent growth model for log drink index scores. The labels mark path coefficients that were constrained across assessment points. Condition = control or experimental group; Intercept $=$ baseline log drink index; Slope = change log drink index; Log Drink Pre-test = log drink index score at pre-test; Log Drink Post-test = log drink index score at post-test; Log Drink FU 1 = log drink index score at six month follow-up; Log Drink FU 2 = log drink index score at one year follow-up.

from the intercept factor to each measurement point to 1 and all paths from the slope factor to each measurement point to $0,7,26$ and 52 this model represents linear growth. By introducing condition as a predictor of intercept and slope, these two latent factors are estimated based on group membership. The path from condition to intercept tests if the experimental group differs from the control group in baseline functioning. This is the main effect of condition. The path from condition to slope tests if individual growth within the experimental group differs from individual growth in the control group. This is the interaction between time and condition we are primarily interested in. The regression weights in Table 3 show that there was a negative but non-significant effect of condition on intercept $(B=-0.120, p=0.39)$ and slope $(B=-0.003, p=0.27)$. This means that the two treatment conditions did not significantly differ with respect to their baseline functioning and change of the drink index over time. So, there was no main effect of condition, and no interaction between time and condition. Furthermore, the main effect of time was also non-significant $(B=0.003, p=0.14)$. The initial level of drink index was significantly different from zero $(B=-0.209, p=0.03)$.

The variances of the latent variables $\cup 0$ and $u 1$ were significantly different from zero $(\operatorname{var}(u 0)=0.369, p<0.001$; var $(u 1)=0.000, p<0.001)$, meaning that there was between-individual variability in the initial level of and change in drink index. These two variables were negatively and significantly correlated $(\operatorname{covar}(v 0, u 1)=-0.002, p=0.04)$, so participants with a higher initial drink index showed a significantly lower increase in drink index over time than participants with a lower level of initial drink index. Finally, the variances of the latent variables $\mathrm{u} 1$-u3 were all significantly different from zero, meaning that there were other factors that contribute to the variability in the drink index scores at the first three time points than those used in the model. 
Table 3

Summary of the parameter estimates in the Latent Growth Model $(\mathrm{N}=107)$

\begin{tabular}{|c|c|c|c|}
\hline & Estimate & S.E. & $p$ \\
\hline \multicolumn{4}{|l|}{ Regression weights } \\
\hline Intercept & -0.209 & 0.097 & 0.031 \\
\hline Slope & 0.003 & 0.002 & 0.141 \\
\hline Condition & -0.120 & 0.138 & 0.385 \\
\hline Condition x Slope & -0.003 & 0.003 & 0.273 \\
\hline \multicolumn{4}{|l|}{ Variances } \\
\hline dl & 0.246 & 0.055 & $* * *$ \\
\hline$d 2$ & 0.266 & 0.052 & $* * *$ \\
\hline d3 & 0.133 & 0.030 & $* * *$ \\
\hline $\mathrm{d} 4$ & 0.038 & 0.055 & 0.492 \\
\hline ט० & 0.369 & 0.071 & $* * *$ \\
\hline ul & 0.000 & 0.000 & 0.011 \\
\hline \multicolumn{4}{|l|}{ Covariances } \\
\hline $\mathrm{v} 0 \leftrightarrow \mathrm{ul}$ & -0.002 & 0.001 & 0.043 \\
\hline
\end{tabular}

Note. Condition = control or experimental group; Intercept = baseline log drink index; Slope = change log drink index.; $* * * p<.001$

\section{DISCUSSION}

This study investigated the effectiveness of the targeted intervention program "Learning to Drink" in a randomized clinical trial design. The aim was to investigate whether this program was successful in changing the targeted cognitive determinants of drinking behavior, such as perception of risk factors and alcohol expectancies. Subsequently, we hypothesized that this change in cognitive determinants would lead to a moderation of the increase in alcohol use and alcohol-related problems in at-risk adolescents. The intervention was effective in changing several of the targeted cognitive determinants: there was a significant increase the perception of risk factors for developing alcohol-related problems and a significant decrease of positive alcohol expectancies for a high dose of alcohol in the experimental group compared with the control group. Despite the changes in these cognitive determinants of drinking, the experimental group did not show a significant difference in decrease of drinking at post-test compared with the control group. The results did not yield support for any differential long term effects of the intervention. So, although the intervention focused on changing determinants of drinking behavior, the subsequent decrease in drinking behavior was not obtained.

The fact that we did not observe the expected change in the subsequent outcome behavior, might reflect that targeting these cognitive determinants alone is not enough to elicit meaningful changes in at-risk youth. Although cognitive behavioral 
skills-based interventions and brief motivational feedback have been proven to be effective in reducing hazardous drinking in (young) adult populations (Larimer \& Cronce, 2002), these strategies might be less effective in at-risk adolescents populations. This reduced effectiveness in these at-risk adolescent populations might be explained by the differential response to alcohol in adolescents and adults. During adolescence not so much the negative sedative effects, but especially the positive arousal effects of drinking are being experienced (NIAAA, 2005). Therefore, these adolescents might feel they do not have sufficient reason for changing their drinking behavior. Furthermore, these intervention strategies might be less effective in these atrisk adolescent populations, because adolescents drinking behavior might be more influenced by other processes than deliberate processes such as alcohol outcome expectancies and awareness of risk factors for developing alcohol problems. It has been suggested that alcohol-induced brain damage in the prefrontal cortex during adolescence might lead to reduced executive functioning (e.g. Crews et al., 2000). In turn, reduced executive functioning might lead to less deliberate control and more automatically induced behavior (Payne, 2005).

However, given a number of limitations, these results should be interpreted with some caution. First, a weakness in the design was the difference in contact hours between the experimental intervention group and the information-only control group. However, it was ensured that the time spent on filling out questionnaires was equal in both groups. Secondly, the absence of a long term effect of the intervention could be due to the fact that the controls also had to fill in numerous questionnaires on their alcohol use and alcohol-related problems. Research has shown that any form of selfmonitoring may affect the behavior that is being monitored (measurement reactivity). Indeed, several studies have shown that monitoring ones alcohol use has moderating impact on the subsequent alcohol use (e.g. Miller et al., 1995). This could serve as a post-hoc explanation for the fact both the control and experimental group did not show the anticipated increase in drinking over time. Since the usual pattern within this age group in the Netherlands is an increase of alcohol use over time (Poelen, Scholte, Engels, Boomsma \& Willemsen, 2005), the fact that no such significant change over time was found might indeed indicate that both the current intervention and the assessment have had a moderating influence on subsequent alcohol use. In this interpretation the assessment of the control group which might have served as a minimal intervention could have been equally effective as the intensive treatment condition. This hypothesis could be tested in follow-up research by including a no or minimal assessment control condition. Thirdly, the absence of a long term effect of the intervention could be due to the fact that the current sample was too small to detect subtle differences in changes in alcohol use or alcohol-related problems between the experimental and control group. It could be that subgroups within this young population profit from this intervention, however this could not be analyzed adequately given the limited power. Indeed, previous research has shown that specific subgroups defined on the 
basis of personality profiles benefit from different intervention strategies (Conrod, Stewart, \& Pihl, 2000). Possibly, that with a larger sample size a differential effectiveness of the intervention for different subgroups could be shown. Yet, the small sample-size seemed to reflect another more fundamental problem of this program; the apparent difficulty of this program to attract at-risk adolescents to participate.

The results of this study suggest that the clinical significance of this intervention was small, given the fact that this intervention could not attract large numbers of at-risk adolescents and was not able to induce meaningful changes in drinking behavior in atrisk adolescents. Although the intervention was effective in changing cognitive determinants of drinking, this did not lead to the expected decrease in drinking behavior. This might suggest that research on the moderation of hazardous drinking in at-risk youth should not only focus on the explicit deliberate processes, but should also take into account that more automatic processes have a strong influence on drinking behavior in at-risk adolescents. Indeed, implicit alcohol associations have been shown to predict a unique part of the variance of subsequent alcohol use in students (Stacy, 1997) and in high-risk adolescents (Stacy, Ames, Sussman, \& Dent, 1996) after controlling for explicit alcohol expectancies. Differences in implicit arousal associations with alcohol dissociated between heavy and light drinkers and explicit alcohol expectancies differed from the implicit association with alcohol (Wiers, Van Woerden, Smulders \& De Jong, 2002). In addition, interventions seem to have differential effect on implicit and explicit alcoholrelated cognition (Wiers et al., 2005). Directly or indirectly influencing these automatically activated alcohol associations might be the next step in the development of targeted intervention programs (Wiers, De Jong, Havermans, \& Jelicic, 2004). 



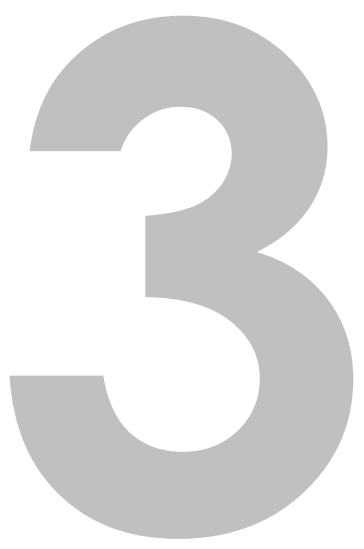

Explicit and implicit alcohol-related cognitions and the prediction of future drinking in adolescents

Published as: Thush, C. \& Wiers, R.W. (2007). Explicit and implicit alcohol-related cognitions and the prediction of future drinking in adolescents. Addictive Behaviors, 32, 1367-1383. 


\section{ABSTRACT}

Both implicit and explicit alcohol-related cognitions might play a role in the early development of addictive behaviors. In this study, the association between both current and prospective alcohol use and implicit and explicit alcohol-related cognitions were measured in two different adolescent age groups ( $N=100 ; 51$ twelve year olds, 49 fifteen year olds). Alcohol-related cognitions were measured on two dimensions (valence and arousal). A new measure, the unipolar Single Target Implicit Association Test (ST-IAT), was used as the implicit measure. A unipolar expectancy questionnaire was used as the explicit measure. Current alcohol use and alcohol use after one year were measured with an alcohol use questionnaire. Abstainers and drinkers differed in both their explicit and implicit alcohol-related cognitions moderated by age and gender. Additionally, a hierarchical regression analysis showed that implicit associations with alcohol added significantly to the prediction of prospective binge drinking, when controlling for grade, gender and explicit alcohol expectancies These results indicate the importance of taking implicit alcohol-related cognitions into account when intervention methods are developed. 
Alcohol-related cognition has been proposed to play an important role in the development and maintenance of addictive behaviors. Explicit alcohol outcome expectancies have shown to be strongly correlated with current alcohol use, predicting up to half of the variance in concurrent alcohol use in cross-sectional studies (Goldman, Del Boca \& Darkes, 1999; Wiers, Hoogeveen, Sergeant \& Gunning, 1997). Explicit alcohol outcome expectancies have also been shown to be predictive of prospective alcohol use (e.g. Goldman \& Darkes, 2004; Stacy, 1997; Stacy, Newcomb \& Bentler, 1991), however it must be noted that the proportion of explained variance is much smaller than in studies that examined concurrent alcohol use (see Jones, Corbin, \& Fromme, 2001). Although explicit alcohol expectancies have been proven successful in predicting future alcohol use and abuse, their assessment has been criticized on a number of methodological and conceptual grounds (Leigh, 1989). Measuring explicit alcohol expectancies requires participants to rely on introspection; however, the question remains whether participants are willing and able to articulate the underlying motivational processes of their behavior (Stacy, 1997; Wiers, van Woerden, Smulders \& De Jong, 2002; cf. Nisbett \& Wilson, 1977). Consequently, several authors proposed the use of implicit or indirect measures (Stacy, 1997; cf. Greenwald \& Banaji, 1995). These implicit measures do not rely on introspective awareness of ones reasons for engaging in certain behavior and are supposed to tap into more automatic underlying motivational processes (Greenwald \& Banaji, 1995; Greenwald, McGhee \& Schwartz, 1998).

Recently, there has been a growing interest into the role of implicit cognitions in the development of addictive behaviors. Implicit cognitions are traces of past experience that mediate behavior in a relatively automatic fashion, whereas explicit cognitions are introspectively accessible cognitions related to more slow deliberate choices (Greenwald \& Banaji, 1995; Strack \& Deutsch, 2004). It is likely that both explicit and implicit alcohol-related cognitions play an important role in the development of addictive behavior, since both implicit and explicit alcohol-related cognitions have been shown to predict a unique part of the variance in current and prospective alcohol use (e.g. Jajodia \& Earleywine, 2003; Stacy, 1997; Wiers et al., 2002). Moreover, implicit and explicit attitudes could originate from different sources. Implicit attitudes may be related to early and affective experiences, whereas explicit attitudes may be based more on recent events (Rudman, 2004).

Until now, research has mainly focused on the development of explicit alcohol cognitions in the early stages of alcohol consumption. It has been proposed that children may have a 'critical period' around the age of 10 in the process of developing outcome expectancies towards alcohol. Around this age, children's expectancies towards alcohol have been shown to shift from primarily negative to a primarily positive point of view (Dunn \& Goldman, 1996, 1998, 2000). This bipolar shift in alcohol expectancies seems to mark the beginning of the initiation of alcohol consumption (Wiers, Gunning, Sergeant, 1998). Later research indicated that positive and negative expectancies may develop in a more parallel fashion (Cameron, Stritzke \& Durkin, 2003). 
Children seem to hold both positive and negative alcohol expectancies simultaneously. When children are young, they primarily report negative alcohol expectancies although they already hold positive alcohol expectancies as well. When children grow older, they report more positive alcohol expectancies next to the negative alcohol expectancies they already had. In other words, children seem to become more ambivalent towards alcohol because of a relative increase in the activation of positive expectancies, which resulted in a balance between the activation of positive and negative expectancies (Cameron et al., 2003).

So far only few scientific attempts have been made at investigating the development of implicit alcohol-related cognitions in the early stages of alcohol consumption. However, we do know more about implicit alcohol-related cognitions once drinking alcohol has become common practice. Using an implicit memory task, implicit alcoholrelated cognition uniquely predicts prospective alcohol use in high risk adolescents next to previous alcohol use, explicit cognitions and impulsive sensation seeking (Stacy, Ames, Sussman \& Dent et al., 1996). Furthermore, Wiers et al. (2002) found using the Implicit Association Task (Greenwald et al., 1998) that heavy drinking college freshmen associated alcohol more strongly with arousal than sedation, whereas light drinkers did not. Therefore, Wiers et al. (2002) have been arguing that the implicit arousal associations could represent a human equivalent of 'incentive sensitization' that develops with repeated alcohol and drug use (Robinson \& Berridge, 1993). Consistently, alcohol expectancy research has shown that older and heavier drinking adolescents showed stronger arousal alcohol expectancies, whereas lighter drinking children showed stronger sedation alcohol expectancies (Dunn \& Goldman, 1998; 2000). However, contrary to what was found in alcohol expectancy research (Dunn \& Goldman, 1998; 2000), both light and heavy drinkers more strongly associated alcohol with negative than with positive outcomes. The finding that heavy drinkers hold both negative and arousal associations has been replicated (De Houwer, Crombez, Koster \& De Beul, 2004; Wiers, van de Luitgaarden, van den Wildenberg \& Smulders, 2005). Yet, the interpretation of this finding is still not entirely clear: it has been proposed that this implicit negative association with alcohol could represent negative experiences with alcohol (cf. Jones \& McMahon, 1996), cultural evaluations or salience asymmetries (Houben \& Wiers, 2006; cf. Rothermund \& Wentura, 2004) or a relative preference for sodas (De Houwer et al., 2004). Although Houben and Wiers (2006) recently showed that salience asymmetries cannot fully account for negative association with alcohol, the questions remains what these implicit negative associations with alcohol actually represent.

Given remaining questions regarding the development of explicit cognitions and implicit alcohol-related cognitions in the early stages of alcohol consumption, it is important to measure explicit and implicit alcohol-related cognitions in the early development of alcohol consumption. Therefore, this study focused on two main questions. The first main question was what the differences would be in implicit and explicit alcohol-related cognitions between adolescents who initiated drinking (drink- 
ers) and those who did not initiate drinking (abstainers). It was expected that drinkers and abstainers would differ in both their explicit and implicit alcohol-related cognitions. More specifically, it was hypothesized that drinkers would show stronger positive and arousal alcohol expectancies and weaker negative and sedation alcohol expectancies than abstainers (cf. Cameron et al., 2003; Dunn \& Goldman 1996, 1998, 2000; Wiers et al., 1997). When one assumes that implicit associations with alcohol partly represent cultural evaluations (Houben \& Wiers, 2006), one would expect that abstainers primarily hold negative associations towards alcohol given the fact that do not have any direct experience either positive or negative with alcohol yet. Additionally, when one assumes that these implicit associations with alcohol represent experiences with alcohol (Wiers, Houben, Smulders, Conrod, \& Jones, 2006; cf. Jones \& McMahon, 1996), one would expect that heavily drinking adolescents with problematic alcohol use would hold stronger negative implicit associations towards alcohol than lighter drinker adolescents. Thus, it seems as though implicit negative associations might develop according to an inverted U-shaped curve, whereas implicit positive associations with alcohol seem to develop in a positive linear relationship. It should be noted that this proposed relationship between the development of drinking and implicit associations is at this point merely theoretical. Since this study was designed to compare abstaining adolescents with (relative light) drinking adolescents, we expected that drinking adolescents would hold weaker negative associations with alcohol and stronger positive associations with alcohol than abstainers. In addition, it was hypothesized that drinkers would show stronger implicit arousal associations and weaker implicit sedation associations with alcohol than abstainers (Wiers et al., 2002). The second main question was which variables would predict prospective drinking after one year. It was hypothesized that besides grade and gender, both explicit and implicit cognitions would predict a unique part of the variance in prospective drinking. Furthermore, it was expected that implicit arousal and implicit negative associations with alcohol would positively predict prospective drinking (cf. Stacy, 1997; Wiers et al., 2002).

Given the fact that Cameron et al. (2003) showed that children can be positive and negative towards alcohol at the same time, we decided to use unipolar measures to assess alcohol-related cognitions in this study. A new measure, the unipolar Single Target Implicit Association Test (ST-IAT), was used as the implicit measure of alcoholrelated cognitions. The ST-IAT is an adapted version of the IAT and has the advantage that no contrast category is used (Wigboldus, 2001, in De Houwer, 2002; Wigboldus, Holland \& van Knippenberg, 2005; cf. Huijding \& De Jong, 2006; Karpinski \& Steinman, 2006). This measure was chosen in order to obtain single associations with alcohol instead of the relative comparison between alcohol and a contrast category (e.g. soft drinks) which is measured with the original IAT. Moreover, this unipolar measure was used in order to measure the association between alcohol and a single attribute (e.g. positive) instead of the association with alcohol on a bipolar scale (e.g. positive vs. negative). A unipolar explicit expectancy questionnaire was used as the explicit meas- 
ure of alcohol-related cognitions to be able to compare implicit and explicit alcoholrelated cognitions. After one year, participants were followed up regarding their alcohol use.

\section{METHOD}

\section{Participants}

One hundred Dutch high school students (49 males) participated. Fifty-one participants were twelve years old (mean age 12.29; $S D=0.46$ ) and 49 were fifteen years old (mean age 15.53; $S D=0.58$ ). Participants were recruited from Dutch grades one and four of secondary education. These two grades were chosen based on a national health survey among high school adolescents which showed a large increase between these grades in the number of adolescent who started drinking alcohol. Participants were regarded as abstainers if they indicated that they did not drink any alcoholic beverages. Participants were regarded as drinkers if they indicated that they normally drank at least one alcoholic consumption per week (see Table 1). Drinkers drank an average of 8.36 Dutch standard alcoholic drinks per week $(S D=8.97$ ) (a standard alcohol serving in the Netherlands contains somewhat less alcohol than a standard English or American standard glass: 10 vs. 14 g). Out of the 57 drinkers, 33 (57.9\%) indicated having one or more binge drinking episodes (five or more Dutch standard drinks on one occasion) in the past two weeks.

\section{Material}

\section{Alcohol Use}

Alcohol use was measured with a shortened version of a self-report Dutch alcohol use questionnaire (Wiers et al, 1997), based on the timeline follow-back method (Sobell \& Sobell, 1990). Self-report questionnaires have been proven to be reliable and valid if the soberness of the participant and confidentiality of data is assured (Sobell \& Sobell, 1990). Both requirements were fulfilled in this study. Participants indicated how many Dutch standard glasses of alcohol they normally consumed for each day of the week. Additionally, they indicated on a 6 point Likert scale on how many occasions they drank five Dutch standard glasses of alcohol or more in the past two weeks (ranging from 'I do not drink' to 'seven times or more'). From these alcohol consumption measures, a quantity-frequency index was calculated (number of drinks per week) and a binge drinking index was calculated (frequency of binges per two week). 


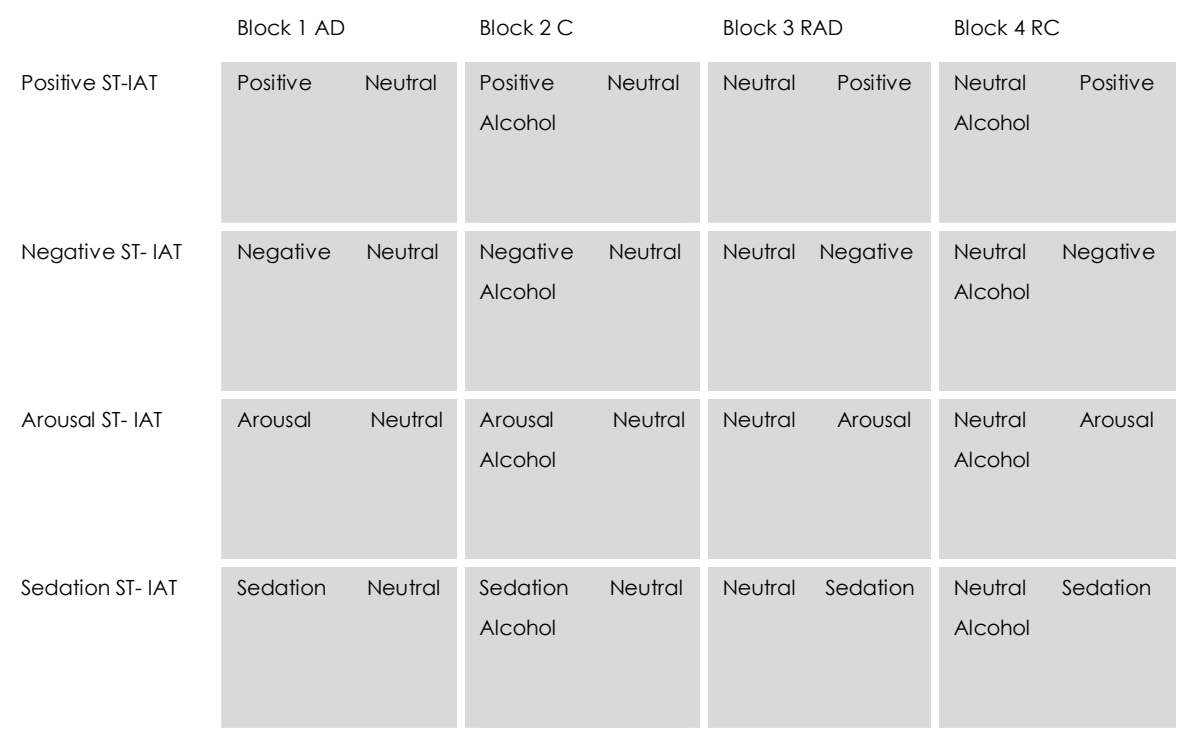

Figure 1. Schematic overview of the block sequence in the four Single Target - Implicit Association Tests. AD = Attribute Discrimination, $\mathrm{C}=$ Combination, $\mathrm{RAD}=$ Reversed Attribute Discrimination, $\mathrm{RC}=$ Reversed Combination.

\section{Implicit Measure}

A new measure, the unipolar Single Target Implicit Association Test (ST-IAT) was used as the implicit measure of alcohol-related cognitions. The single target IAT (ST- IAT) is a new variant of the IAT (Greenwald et al., 1998) that does not require the use of target contrast category (in case of alcohol often soft-drinks).

In the ST-IAT, participants categorized words as quickly as possible without making too many mistakes into different categories by pressing a left or right response-button. The test consisted of four blocks: the attribute discrimination block, the combination block, the reversed attribute discrimination block and the reversed combination block (see Figure 1). During the attribute discrimination block and the reversed attribute discrimination block, participant categorize stimulus words into one attribute category (e.g. positive) or the other (e.g. neutral). During the combination and reversed combination block, the target category (e.g. alcohol) is paired with one of the attributes (i.e. positive or neutral). For someone who has a very strong association between the target (e.g. alcohol) and one of the attribute categories (e.g. positive), the compatible combination block (alcohol and positive on the same side) will be significantly easier than the incompatible reversed combination block (alcohol and positive on different sides). The difference score between the reaction times of these two combined blocks is the so-called IAT-effect and gives an indication of strength of the association between target (e.g. alcohol) and the attribute (e.g. positive) (Greenwald et al., 1998). 
Thus, a larger IAT-effect indicates a stronger the association between the target (e.g. alcohol) and the attribute (e.g. positive).

In this study four ST-IATs (alcohol-positive, alcohol-negative, alcohol-arousal, alcohol-sedation) were used in balanced order. The ST-IATs were programmed in ERTS 3.18 (Beringer, 1996). Words were presented in black small font (14-point) in the middle of the screen. Feedback appeared in red mid font letters (16-point) 1 inch below the stimuli words. The category words were always presented at the top of the screen, appropriately positioned on the left or the right side of the screen, depending on the required response. The words used (see Appendix) were matched on number of letters, syllables, familiarity, and on valence and arousal values. In total, these four ST-IATs consisted of 16 blocks; each consisted of two attribute discrimination blocks and two combined blocks. Each attribute discrimination block consisted of 24 trials and each combined block consisted of 48 trials. The interstimulus interval was $250 \mathrm{~ms}$. Words were selected randomly for each participant. The internal consistency of each of these four ST-IATs was acceptable (see Table 2).

The D-2SD penalty score for practice and test was chosen as the main reaction time measure, because Greenwald, Nosek and Banaji (2003) recently recommended using a new "D-algorithm" that includes all trials (including the practice phases), gives an error-penalty to the reaction times on erroneous responses, and divides the outcome with a personalized SD of the combination phases. This particular D-measure was chosen, because in the calculation of the recommended new algorithm score a standard deviation of $300 \mathrm{~ms}$ is assumed (Greenwald et al, 2003) and at this point there is no information available to determine whether this is a plausible assumption when testing adolescents. Following the recommendation of Greenwald et al. (2003), the effects are calculated with the D-measure, but in Table 1 the IAT effects are given in ms. Main outcomes were also calculated with the original scoring algorithm and the pattern of results was the same.

\section{Explicit Measure}

The explicit measure of alcohol-related cognitions was a 24 unipolar item questionnaire representing an explicit version of the exact same words as used in the implicit tests (cf. Wiers et al. 2002; 2005). Each item consisted of a statement on drinking alcohol (for example: 'drinking alcohol makes me feel energetic'). Participants indicated the extent to which they (dis)agreed with each item on a $110 \mathrm{~mm}$ unmarked Visual Analog Scale (VAS). The questionnaire consisted of four scales: a positive, a negative, an arousal and a sedation scale. The internal consistency of the positive and negative scale was acceptable (see Table 2). After removing two deviating items from the arousal and sedation scale, the internal consistency of these scales was moderate (see Table 2). 
Table 1

Means and Standard Deviations of the Explicit and Implicit Dependent Variables and Alcohol Use

\begin{tabular}{|c|c|c|c|c|c|c|c|c|}
\hline & \multicolumn{4}{|l|}{ Grade 1} & \multicolumn{4}{|l|}{ Grade 4} \\
\hline & \multicolumn{2}{|l|}{ Boys } & \multicolumn{2}{|l|}{ Girls } & \multicolumn{2}{|l|}{ Boys } & \multicolumn{2}{|l|}{ Girls } \\
\hline & $\begin{array}{l}\text { Abstainers } \\
(N=19)\end{array}$ & $\begin{array}{l}\text { Drinkers } \\
(N=10)\end{array}$ & $\begin{array}{l}\text { Abstainers } \\
(N=15)\end{array}$ & $\begin{array}{l}\text { Drinkers } \\
(N=7)\end{array}$ & $\begin{array}{l}\text { Abstainers } \\
(N=5)\end{array}$ & $\begin{array}{l}\text { Drinkers } \\
(N=15)\end{array}$ & $\begin{array}{l}\text { Abstainers } \\
(N=4)\end{array}$ & $\begin{array}{l}\text { Drinkers } \\
(N=25)\end{array}$ \\
\hline Expl. Pos. Expec. & $\begin{array}{l}3.46 \\
(2.33)\end{array}$ & $\begin{array}{l}5.01 \\
(2.05)\end{array}$ & $\begin{array}{l}4.02 \\
(1.71)\end{array}$ & $\begin{array}{l}5.47 \\
(1.03)\end{array}$ & $\begin{array}{l}4.56 \\
(2.75)\end{array}$ & $\begin{array}{l}7.17 \\
(1.88)\end{array}$ & $\begin{array}{l}4.53 \\
(3.14)\end{array}$ & $\begin{array}{l}6.92 \\
(1.96)\end{array}$ \\
\hline Expl. Neg. Expec. & $\begin{array}{l}4.11 \\
(2.71)\end{array}$ & $\begin{array}{l}3.88 \\
(3.00)\end{array}$ & $\begin{array}{l}4.57 \\
(2.24)\end{array}$ & $\begin{array}{l}2.61 \\
(1.70)\end{array}$ & $\begin{array}{l}2.07 \\
(2.02)\end{array}$ & $\begin{array}{l}2.14 \\
(1.33)\end{array}$ & $\begin{array}{l}4.52 \\
(2.37)\end{array}$ & $\begin{array}{l}2.22 \\
(1.60)\end{array}$ \\
\hline Expl. Aro. Expec. & $\begin{array}{l}5.08 \\
(2.75)\end{array}$ & $\begin{array}{l}4.81 \\
(1.48)\end{array}$ & $\begin{array}{l}5.27 \\
(1.90)\end{array}$ & $\begin{array}{l}4.49 \\
(1.17)\end{array}$ & $\begin{array}{l}3.97 \\
(0.97)\end{array}$ & $\begin{array}{l}6.29 \\
(2.17)\end{array}$ & $\begin{array}{l}6.04 \\
(1.72)\end{array}$ & $\begin{array}{l}6.50 \\
(1.92)\end{array}$ \\
\hline Expl. Sed. Expec. & $\begin{array}{l}3.22 \\
(2.40)\end{array}$ & $\begin{array}{l}4.36 \\
(1.78)\end{array}$ & $\begin{array}{l}2.62 \\
(1.26)\end{array}$ & $\begin{array}{l}3.21 \\
(1.18)\end{array}$ & $\begin{array}{l}4.07 \\
(1.65)\end{array}$ & $\begin{array}{l}4.45 \\
(1.43)\end{array}$ & $\begin{array}{l}3.56 \\
(1.65)\end{array}$ & $\begin{array}{l}2.56 \\
(1.69)\end{array}$ \\
\hline Impl. Pos. Assoc. & $\begin{array}{l}-34.70 \\
(136.81)\end{array}$ & $\begin{array}{l}-21.08 \\
(73.56)\end{array}$ & $\begin{array}{l}-53.05 \\
(118.53)\end{array}$ & $\begin{array}{l}-51.68 \\
(170.47)\end{array}$ & $\begin{array}{l}-22.47 \\
(122.30)\end{array}$ & $\begin{array}{l}38.04 \\
(152.29)\end{array}$ & $\begin{array}{l}-84.97 \\
(100.79)\end{array}$ & $\begin{array}{l}-14.81 \\
(118.91)\end{array}$ \\
\hline Impl. Neg. Assoc. & $\begin{array}{l}-13.55 \\
(151.04)\end{array}$ & $\begin{array}{l}47.58 \\
(185.37)\end{array}$ & $\begin{array}{l}36.88 \\
(130.12)\end{array}$ & $\begin{array}{l}43.52 \\
(110.28)\end{array}$ & $\begin{array}{l}134.85 \\
(201.31)\end{array}$ & $\begin{array}{l}-11.70 \\
(94.99)\end{array}$ & $\begin{array}{l}70.94 \\
(114.21)\end{array}$ & $\begin{array}{l}-12.70 \\
(105.18)\end{array}$ \\
\hline Impl. Aro. Assoc. & $\begin{array}{l}-70.39 \\
(156.21)\end{array}$ & $\begin{array}{l}30.73 \\
(166.29)\end{array}$ & $\begin{array}{l}-9.78 \\
(95.14)\end{array}$ & $\begin{array}{l}-83.86 \\
(98.41)\end{array}$ & $\begin{array}{l}123.04 \\
(96.42)\end{array}$ & $\begin{array}{l}94.59 \\
(126.89)\end{array}$ & $\begin{array}{l}-21.70 \\
(90.71)\end{array}$ & $\begin{array}{l}13.81 \\
(102.90)\end{array}$ \\
\hline Impl. Sed. Assoc. & $\begin{array}{l}-16.86 \\
(91.78)\end{array}$ & $\begin{array}{l}-26.05 \\
(108.29)\end{array}$ & $\begin{array}{l}-72.35 \\
(130.04)\end{array}$ & $\begin{array}{l}-46.42 \\
(62.83)\end{array}$ & $\begin{array}{l}67.83 \\
(196.36)\end{array}$ & $\begin{array}{l}-28.62 \\
(81.96)\end{array}$ & $\begin{array}{l}-71.967 \\
(120.14)\end{array}$ & $\begin{array}{l}-9.40 \\
(97.84)\end{array}$ \\
\hline Alc. Use & $\begin{array}{l}0 \\
(0)\end{array}$ & $\begin{array}{l}3.05 \\
(1.64)\end{array}$ & $\begin{array}{l}0 \\
(0)\end{array}$ & $\begin{array}{l}2.21 \\
(1.82)\end{array}$ & $\begin{array}{l}0 \\
(0)\end{array}$ & $\begin{array}{l}16.33 \\
(12.14)\end{array}$ & $\begin{array}{l}0 \\
(0)\end{array}$ & $\begin{array}{l}7.42 \\
(6.04)\end{array}$ \\
\hline
\end{tabular}

Note. All explicit expectancy scores were average scores on an unmarked $110 \mathrm{~mm}$ Visual Analogue Scales; all implicit association scores were reaction time difference scores in $\mathrm{ms}$. between the incompatible and compatible block on the Single Target Implicit Association Test; Expl. Pos. Expec. = Explicit Positive Expectancies; Expl. Neg. Expec. = Explicit Negative Expectancies; Expl. Aro. Expec. $=$ Explicit Arousal Expectancies; Expl. Sed. Expec. $=$ Explicit Sedation Expectancies; Impl. Pos. Assoc. $=$ Implicit Positive Associations; Impl. Neg. Assoc. = Implicit Negative Associations; Impl. Aro. Assoc. = Implicit Arousal Associations; Impl. Sed. Assoc. = Implicit Sedation Associations; Alc. Use = number of standard drinks per week at TO

\section{Procedure}

After this study was approved by the ethical committee of the research institute, participants were recruited. After active consent from participants and parents, participants were tested individually at school in a separate test room during school time. After a short introduction, the ST-IATs were administered on a laptop with a separate response device. The four ST-IATs consisted of a total of 16 blocks with a short break after the $8^{\text {th }}$ block. The instructions were given on the computer screen preceding each task. Throughout the trails, participants received feedback on their screen in red letters (incorrect response: "ERROR", <300ms: TOO SLOW', >3000 ms: 'TOO FAST'). After each block, the participants received feedback on their performance in the form of average reaction times and errors. The total task required approximately 15 minutes. The implicit measure was administered before the explicit measure, because of possible carry-over effects which are stronger when the explicit test is administered first (Bosson, Swann \& Pennebaker, 2000). Subsequently, the participants filled out the explicit measure of 
Table 2

Pearson Correlation and Cronbach Alphas for Implicit and Explicit Cognition and Alcohol Use

\begin{tabular}{|c|c|c|c|c|c|c|c|c|c|c|c|c|}
\hline & \# Items & $\alpha$ & 1 & 2 & 3 & 4 & 5 & 6 & 7 & 8 & 9 & 10 \\
\hline 1. Expl. Pos. Expec. & 6 & .87 & - & & & & & & & & & \\
\hline 2. Expl Neg. Expec. & 6 & .59 & $.43^{* *}$ & - & & & & & & & & \\
\hline 3. Expl. Aro. Expec. & 4 & .81 & $.34^{* *}$ & .15 & - & & & & & & & \\
\hline 4. Expl. Sed. Expec. & 4 & .55 & -.04 & .16 & $-.22^{*}$ & - & & & & & & \\
\hline 7. Impl Aro. Assoc. & 2 & .46 & .16 & .03 & .13 & .04 & .16 & -.03 & - & & & \\
\hline 8. Impl Sed. Assoc. & 2 & .43 & -.02 & -.01 & -.06 & .20 & .15 & .10 & .07 & - & & \\
\hline 9. Alcohol Use TO & 7 & .63 & $.53^{* *}$ & $-.33^{* *}$ & $.22^{*}$ & .04 & $.25^{*}$ & -.08 & $.26^{*}$ & .06 & - & \\
\hline
\end{tabular}

Note. All explicit expectancy scores were average scores on an unmarked $110 \mathrm{~mm}$ Visual Analogue Scales; all implicit association scores were D 2-SD reaction time scores on the Single Target Implicit Association Test; Expl. Pos. Expec. = Explicit Positive Expectancies; Expl. Neg. . Expec. = Explicit Negative Expectancies; Expl. Aro. Expec. = Explicit Arousal Expectancies; Expl. Sed. Expec. = Explicit Sedation Expectancies; Impl. Pos. Assoc. = Implicit Positive Associations; Impl. Neg. Assoc. = Implicit Negative Associations; Impl. Aro. Assoc. = Implicit Arousal Associations; Impl. Sed. Assoc. $=$ Implicit Sedation Associations; Alcohol Use TO = combined number of standard drinks for each day of the week at TO; Alcohol Use $\mathrm{T} 1$ = combined number of standard drinks for each day of the week after one year $\mathrm{T} 1 .{ }^{*} \mathrm{p}<.05, \mathrm{two}$-tailed. ${ }^{* *} \mathrm{p}<.01$, two-tailed.

alcohol related-cognitions. The alcohol use questionnaire was administered last, in order to avoid any interference between having to report ones alcohol use and the measures of implicit and explicit alcohol-related cognitions. The total experiment took approximately 25 minutes for each participant, after which the participant received their monetary incentive $(€ 2,50)$. Approximately one year later, the participants received a letter on their home address with the question to fill out the enclosed alcohol use questionnaire. The confidentiality of the data was guaranteed. Participants were requested to return the filled out questionnaire with an enclosed prepaid envelop within one week to receive an additional reward ( $€ 5$ voucher). Eighty one of the 100 participants returned a filled out follow-up alcohol use questionnaire. The 19 nonresponders scored somewhat higher on the binge-drinking index than the 81 responders at baseline, $t(22.52)=-1.88, p=.07$.

\section{RESULTS}

This study focused on two main questions. The first question concerned the difference in explicit and implicit alcohol-related cognitions between abstainers and drinkers (crosssectional). The second question concerned which factors in drinkers predicted their prospective drinking behavior after one year. The initial alpha level was set at .05 for all analyses. This decision was made to ensure an optimal trade-off between completeness (not leaving out possibly interesting effects) and the correctness (restricting Type-II error) given the exploratory nature of the data. However, it should be noted that 
especially borderline significant effects need to be interpreted with caution given the fact that multiple testing leads to a larger chance at a Type II error.

\section{Difference in alcohol-related cognitions between abstainers and drinkers (cross-sectional)}

\section{Explicit Measures}

POSITIVE EXPECTANCIES. A 2 (Drinker) $\times 2$ (Gender) $\times 2$ (Grade) ANOVA for positive alcohol expectancies revealed a significant effect for $\operatorname{Grade}(F(3,96)=9.28, p<.001)$ and Drinker $(F(3,96)=15.87, p<.001)$. Fifteen year olds generally had stronger positive alcohol expectancies than twelve year olds ( $\dagger(98)=-5.44, p<.001$ ) and drinkers generally had stronger positive alcohol expectancies than abstainers $(\dagger(98)=-6.10, p<$ .001) (see Table 1).

NEGATIVE EXPECTANCIES. A 2 (Drinker) x 2 (Gender) $\times 2$ (Grade) ANOVA for negative alcohol expectancies revealed a significant Gender $X$ Drinker interaction effect ( $F$ $(6,93)=4.04, p<.05)$. Only among girls, drinkers and abstainers significantly differed from each other $(t(29.38)=3.89, p<.001)$. Female abstainers had stronger negative alcohol expectancies than female drinkers (see Table 1).

AROUSAL EXPECTANCIES. A 2 (Drinker) $\times 2$ (Gender) $\times 2$ (Grade) ANOVA for arousal alcohol expectancies revealed a significant Grade X Drinker interaction effect $(F(6,93)$ $=4.13, p<.05)$. Only among fifteen year olds, drinkers and abstainers significantly differed $(F(1,47)=4.58, p<.05)$. Fifteen year old drinkers had stronger arousal alcohol expectancies than fifteen year old abstainers (see Table 1).

SEDATION EXPECTANCIES. A 2 (Drinker) $\times 2$ (Gender) $\times 2$ (Grade) ANOVA for sedating alcohol expectancies revealed a significant Gender effect $(F(3,96)=12.06, p<$ $.01)$. Boys generally had stronger sedating alcohol expectancies than girls $(t(89.59)=$ 3.33, $p<.01$ ) (see Table 1).

\section{Implicit measures}

The average overall percentage of errors made on the ST-IAT was $17.69 \%$ (SD $=8.51$ ). This number is relatively high, but could be explained by the fact that this task still is relatively difficult for young adolescents. To determine if every participant should be included for further analysis, we used 3 different criteria: the average overall percentage of errors, the percentage of responses under the $300 \mathrm{~ms}$. and the percentage of responses over the $10.000 \mathrm{~ms}$. (Greenwald et al., 2003). In total 3 participants were excluded from further analysis because they exceeded at least one of these criteria with more than 2 SDs.

IMPLICIT POSITIVE ASSOCIATIONS. A 2 (Drinker) $\times 2$ (Gender) $\times 2$ (Grade) ANOVA for implicit positive associations with alcohol revealed a significant Grade X Drinker interaction effect $(F(3,96)=4.85, p<.05)$. Only among fifteen year olds, drinkers and abstainers significantly differed in their implicit positive cognitions towards alcohol $(F(1,46)=$ 
$5.19, p<.05)$. Fifteen year old drinkers had stronger implicit positive associations with alcohol than fifteen year old abstainers (see Table 1). Fifteen year old drinkers showed a significant ST-IAT effect associating alcohol more strongly with positive than neutral attributes $(\dagger(14)=2.16, p<.05)$.

IMPLICIT NEGATIVE ASSOCIATIONS. A 2 (Drinker) $\times 2$ (Gender) $\times 2$ (Grade) ANOVA for implicit negative associations revealed a significant Grade $X$ Drinker interaction effect $(F(6,90)=4.19, p<.05)$. Only among fifteen year olds, drinkers and abstainers significantly differed in their implicit negative cognitions towards alcohol $(F(1,46)=$ $7.98, p<.01$ ). Fifteen year old abstainers had stronger implicit negative associations with alcohol than fifteen year old drinkers (see Table 1). Fifteen year old abstainers showed a borderline significant ST-IAT effect associating alcohol more strongly with negative than neutral attributes $(t(8)=2.15, p=.06)$.

IMPLICIT AROUSAL ASSOCIATIONS. A 2 (Drinker) $\times 2$ (Gender) $\times 2$ (Grade) ANOVA for implicit arousal associations with alcohol revealed a borderline significant Grade $X$ Gender X Drinker interaction effect $(F(7,89)=3.89, p=.05)$. A 2 (Drinker) X 2 (Gender) ANOVA only revealed a significant Gender $X$ Drinker interaction effect for implicit arousal associations among twelve year olds $(F(3,45)=7.30, p<.05)$. Only among the twelve year olds, boys and girls differed significantly in their alcohol-arousal associations. Among twelve year old boys, drinkers significantly differed from abstainers ( $F$ ( 1 , $26)=4.345, p<.05)$. Twelve year old drinking boys did associate alcohol more strongly with arousal than twelve year old abstaining boys (see Table 1). However, among twelve year old girls, drinkers differed from abstainers at borderline significance $(F(1,21)$ $=3.08, p=.10$ ). Contrary to twelve year old boys, twelve year old female drinkers had a tendency to have weaker arousal associations with alcohol than twelve year old female abstainers (see Table 1).

IMPLICIT SEDATION ASSOCIATIONS. A 2 (Drinker) $\times 2$ (Gender) $\times 2$ (Grade) ANOVA for implicit sedation associations with alcohol revealed a significant Gender $X$ Drinker interaction effect $(F(3,96)=3.36, p=.07)$. Only among girls, drinkers differed from abstainers at borderline significance $(F(1,48)=3.38, p=.07)$. Female drinkers had stronger implicit sedation associations with alcohol than female abstainers (see Table 1). Female drinkers did not show a significant ST-IAT effect for implicit sedation association with alcohol.

\section{Factors predicting prospective drinking behavior after one year}

The same three participants were excluded from the analysis because they exceeded at least one of the set criteria with more than 2 SDs. In addition, one other participant was excluded from the regression analysis (Cook's distance $>1$ ). We chose to use prospective binge drinking after one year as dependent variable, given the fact that adolescent binge drinking causes severe damage and long term consequences in the 
Table 3

Summary of Multivariate Regression Analysis for Variables Predicting Binge Drinking After One Year $(N=77)$

\begin{tabular}{|c|c|c|c|c|c|}
\hline \multirow[b]{2}{*}{ Variable } & \multicolumn{2}{|c|}{ Cumulative } & \multicolumn{3}{|c|}{ Simultaneous } \\
\hline & $\mathrm{R}^{2}$ & $\Delta R^{2}$ & $B$ & SE B & $\beta$ \\
\hline Grade & & & 0.38 & 0.16 & $.25^{*}$ \\
\hline Gender & .25 & & -0.40 & 0.41 & -.25 \\
\hline Expl. Pos. Expec. & & & 0.04 & 0.04 & .11 \\
\hline Expl. Neg. Expec. & & & -0.10 & 0.04 & $-.28 * *$ \\
\hline Expl. Aro. Expec. & & & -0.02 & 0.05 & -.05 \\
\hline Expl. Sed. Expec. & .32 & .07 & 0.06 & 0.04 & .14 \\
\hline Impl. Pos. Assoc. & & & 0.60 & 0.18 & $.43^{* *}$ \\
\hline Impl. Neg. Assoc. & & & 0.04 & 0.23 & .02 \\
\hline Impl. Aro. Assoc. & & & 0.86 & 0.30 & $.53^{* *}$ \\
\hline Impl. Sed. Assoc. & .42 & $.10^{*}$ & -0.17 & 0.14 & -.10 \\
\hline Grade X Impl. Neg. Assoc. & & & -1.80 & 0.42 & $-.67^{* *}$ \\
\hline Gender X Impl. Pos. Assoc. & & & -0.50 & 0.25 & -.26 \\
\hline Gender X Impl. Neg. Assoc. & & & 0.03 & 0.31 & .02 \\
\hline Gender X Impl. Aro. Assoc. & & & -0.46 & 0.34 & -.24 \\
\hline Gender X Expl. Aro. Expec. & & & 0.11 & 0.07 & .46 \\
\hline Grade X Gender X Impl. Neg. Assoc. & .63 & $.21^{* *}$ & 2.22 & 0.55 & $.66^{* *}$ \\
\hline
\end{tabular}

Note. $R^{2}$ and $\Delta R^{2}$ are from hierarchical models in which preceding effects were entered first; the $B, S E B$ and $\beta$ are from a simultaneous model. Expl. Pos. Expec. = Explicit Positive Expectancies; Expl. Neg. Expec. = Explicit Negative Expectancies; Expl. Aro. Expec. $=$ Explicit Arousal Expectancies; Expl. Sed. Expec. $=$ Explicit Sedation Expectancies; Impl. Pos. Assoc. = Implicit Positive Associations; Impl. Neg. Assoc. = Implicit Negative Associations; Impl. Aro. Assoc. = Implicit Arousal Associations; Impl. Sed. Assoc. = Implicit Sedation Associations. $\mathrm{p}<.05 *$ ** $\mathrm{p}<.01$

adolescent brain (e.g., Crews, Braun, Hoplight, Switzer \& Knapp, 2000; White, Ghia, Levin Swartzwelder, 2000) and that binge drinking had a more normal distribution than other drinking variables. We decided not to conduct a logistic regression predicting binge drinking after one year, because of the loss of relevant information regarding the amount of binging and the relative small number of participants included in the analysis. Instead, a stepwise hierarchical procedure was used for the multiple regression analysis. In Step 1, gender and grade were entered as background variables into the regression equation. In Step 2, the four explicit measures were added to the regression model. In Step 3, the four implicit measures were added to the regression. In Step 4 all two-way and three-way interaction were added. To obtain the final model, all threeway interactions with a $p$ value above .30 were removed from the regression equation. Subsequently, all the two-way interactions with a $p$ value above the .30 were excluded from further analysis. By using this procedure an optimal trade-off between power (including all interactions reduces power) and the completeness (not leaving out important interactions) was ensured. The final model is represented in Table 3.

The hierarchical regression model revealed that the implicit measures (as a group) significantly added to the prospective prediction of binge drinking $\left(\Delta R^{2}=.10, p=.04\right)$, whereas the explicit measures (as a group) did not $\left(\Delta R^{2}=.07, p=.14\right.$ ) (see Table 3). 


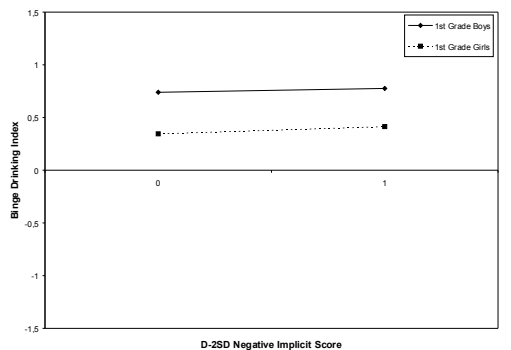

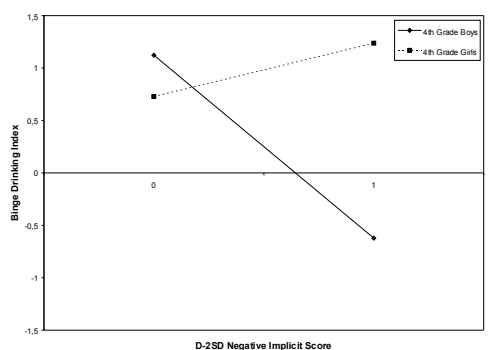

Figure 2. Implicit negative associations predict prospective binge drinking in interaction with grade and gender. The binge drinking index represents on how many occasions five glasses of alcohol or more were consumed in the past two weeks (ranging from 0 = 'I do not drink' to 6 = 'seven times or more'). The D-2SD implicit negative score represents a standardized difference score between the reaction times in the combination and the reversed combination block. A D-2SD implicit negative score of 0 indicates an absent negative association with alcohol as compared with neutral, whereas a D-2SD implicit negative score of one indicates a strong negative association with alcohol compared with neutral. Figure $2 \mathrm{~A}$ indicates that in the first grade implicit negative alcohol associations are not related to prospective binge drinking. To the contrary, Figure $2 \mathrm{~B}$ indicates that in the fourth grade stronger negative alcohol associations negatively predict prospective binge drinking in boys but not in girls.

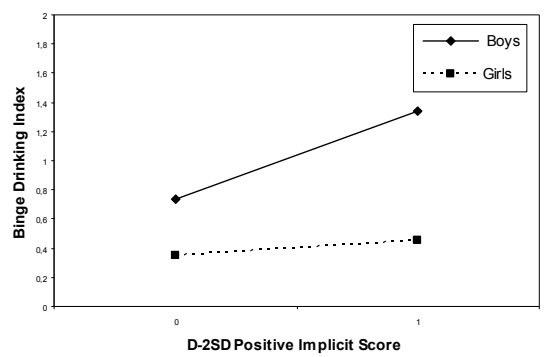

Figure 3. Implicit positive associations predict prospective binge drinking in interaction with gender. The binge drinking index represents on how many occasions five glasses of alcohol or more were consumed in the past two weeks (ranging from $0=$ 'I do not drink' to $6=$ 'seven times or more'). The D-2SD implicit positive score represents a standardized difference score between the reaction times in the combination and the reversed combination block. A D-2SD implicit positive score of zero indicates an absent positive association with alcohol as compared with neutral, whereas a D-2SD implicit positive score of one indicates a strong negative association with alcohol compared with neutral. As is shown, stronger implicit positive alcohol associations positively predict prospective binge drinking in boys but not in girls.

Furthermore, the final model revealed that grade, explicit negative alcohol expectancies, implicit positive associations and implicit arousal association significantly predicted binge drinking after one year (see Table 3). Additionally, the three-way interaction between grade, gender and implicit negative alcohol associations predicted binge drinking after one year significantly (see Table 3). Follow-up analyses indicated that with increasingly stronger negative implicit alcohol associations fourth grade boys engaged 
in less binge drinking, whereas fourth grade girls did not. No such interaction effect was found in the first grade (see Figure 2). Finally, it must be noted that the interaction between gender and positive alcohol associations was borderline significant $(t(60)=-$ 2.00, $p=.05$ ). With increasingly strong positive implicit associations with alcohol, boys displayed a stronger increase in binge drinking compared to girls (see Figure 3).

Additionally, the longitudinal analysis was repeated controlling for baseline binge drinking. Generally the pattern of results remained the same, although the implicit measures did not significantly add to the prediction of prospective binge drinking (see Table 4), which is not surprising, given the limited power of this analysis. Note that it is a different question whether the change in binge drinking can be predicted by implicit and explicit alcohol-related cognitions or binge drinking itself can be predicted by implicit and explicit alcohol-related cognitions. The question that we primarily tried to answer in this study was which cognitive processes might underlie prospective binge drinking. Therefore it seemed most relevant to focus on cognitive factors predicted prospective binge drinking.

Table 4

Summary of Multivariate Regression Analysis for Variables Predicting Binge Drinking After One Year $(\mathrm{N}=77)$

\begin{tabular}{|c|c|c|c|c|c|}
\hline & \multicolumn{2}{|c|}{ Cumulative } & \multicolumn{3}{|c|}{ Simultaneous } \\
\hline Variable & $\mathrm{R}^{2}$ & $\Delta R^{2}$ & $B$ & SE B & $\beta$ \\
\hline Baseline Binge Drinking & & & 0.16 & 0.09 & .18 \\
\hline Grade & & & 0.34 & 0.16 & $.22^{*}$ \\
\hline Gender & $.40^{* *}$ & & -0.41 & 0.40 & -.26 \\
\hline Expl. Pos. Expec. & & & 0.02 & 0.04 & .05 \\
\hline Expl. Neg. Expec. & & & -0.09 & 0.04 & $-.25^{*}$ \\
\hline Expl. Aro. Expec. & & & -0.02 & 0.49 & -.05 \\
\hline Expl. Sed. Expec. & .42 & .02 & 0.06 & 0.04 & .13 \\
\hline Impl. Pos. Assoc. & & & 0.51 & 0.18 & $.36^{* *}$ \\
\hline Impl. Neg. Assoc. & & & 0.01 & 0.22 & .01 \\
\hline Impl. Aro. Assoc. & & & 0.75 & 0.30 & $.46^{*}$ \\
\hline Impl. Sed. Assoc. & .47 & .06 & -0.17 & 0.14 & -.11 \\
\hline Grade X Impl. Neg. Assoc. & & & -1.60 & 0.43 & $-.60 * *$ \\
\hline Gender X Impl. Pos. Assoc. & & & -0.39 & 0.25 & -.20 \\
\hline Gender X Impl. Neg. Assoc. & & & 0.12 & 0.31 & .06 \\
\hline Gender X Impl. Aro. Assoc. & & & -0.37 & 0.33 & -.20 \\
\hline Gender X Expl. Aro. Expec. & & & 0.11 & 0.07 & .48 \\
\hline Grade X Gender X Impl. Neg. Assoc. & .64 & $.16^{* *}$ & 1.96 & 0.57 & $.57^{* *}$ \\
\hline
\end{tabular}

Note. $R^{2}$ and $\Delta R^{2}$ are from hierarchical models in which preceding effects were entered first; the $B, S E B$ and $\beta$ are from a simultaneous model. Expl. Pos. Expec. = Explicit Positive Expectancies; Expl. Neg. Expec. = Explicit Negative Expectancies; Expl. Aro. Expec. = Explicit Arousal Expectancies; Expl. Sed. Expec. = Explicit Sedation Expectancies; Impl. Pos. Assoc. = Implicit Positive Associations; Impl. Neg. Assoc. = Implicit Negative Associations; Impl. Aro. Assoc. = Implicit Arousal Associations; Impl. Sed. Assoc. = Implicit Sedation Associations. $\mathrm{p}<.05 * * \mathrm{p}<.01$ 


\section{DISCUSSION}

This study focused on two main questions. The first question concerned the (crosssectional) difference in explicit and implicit alcohol-related cognitions between abstainers and drinkers. It was found that higher grade and heavier drinking were associated with stronger implicit and explicit positive alcohol-related cognitions, weaker implicit negative alcohol-related cognitions and stronger explicit arousal alcoholrelated cognitions. For other alcohol-related cognitions, interactions were found between gender and drinking-status (explicit negative alcohol-related cognitions, implicit arousal alcohol-related cognitions and implicit and explicit sedation alcohol-related cognitions). The second question concerned which factors predicted binge drinking after one year. The hierarchical regression analyses revealed that overall the implicit measures significantly added to the prediction of binge drinking after one year, whereas the explicit measures (as a group) did not. However, the final regression model revealed that explicit negative alcohol expectancies next to grade and implicit arousal associations predicted binge drinking after one year. Additionally, a three-way interaction between grade, gender and negative implicit associations significantly predicted binge drinking after one year. Noteworthy, a two-way interaction between gender and implicit positive associations predicted prospective binge drinking at borderline significance.

Concerning the first question, the results on the explicit measures are generally in line with what was found in previous research (Cameron et al., 2003, Dunn \& Goldman, $1996 ; 1998 ; 2000)$. Subsequently, although it appears that the implicit measures follow the pattern of the explicit measure, there is an important difference. Only in implicit arousal associations a difference between young drinking boys and young abstaining boys was found. Since this was the only difference found in the young age group, this might indicate that young drinking boys consume alcohol because of associated arousal effects. Furthermore, these young male drinkers might be guided implicitly by these arousal associations with alcohol in their future drinking behavior. Indeed, the results showed that implicit arousal associations significantly predicted prospective binge drinking, but this was not specific for boys.

Concerning the second question, the role of explicit alcohol expectancies in predicting binge drinking after one year may be different and smaller than previously was assumed. First, the role of explicit alcohol expectancies may be smaller, because the hierarchical regression model revealed that overall the explicit measures as a group did not add significantly to the prediction of binge drinking after one year. Additionally, the role of explicit alcohol expectancies may be different, because rather than positive or arousal alcohol expectancies (Dunn \& Goldman, 1996; 1998; 2000), negative alcohol expectancies significantly predicted binge drinking after one year in the final regression model. This is in line with the notion that negative expectancies play a vital role in the preventive approach of affecting current and future drinking (Jones et al., 2001). 
Moreover, the results show that implicit alcohol associations are important in the early development of drinking. The hierarchical regression model revealed that the implicit measures significantly added to the prediction of binge drinking after one year when controlling for gender, grade and explicit expectancies. This is consistent with previous research on the influence of implicit and explicit alcohol cognitions in older adolescents (Stacy et al., 1996).

In addition, one conclusion is that the influence of implicit alcohol-related valence associations on subsequent drinking behavior seems to be dependent of factors like gender and grade; a three-way interaction between grade, gender and negative implicit associations (and a two-way interaction between gender and implicit positive associations at borderline significance) predicted binge drinking after one year. To the contrary, implicit alcohol arousal associations and explicit negative alcohol expectancies seem to influence drinking behavior more generally across ages and gender as the final regression model showed. Although the general nature of the predictive value of implicit arousal associations and explicit negative alcohol expectancies are similar to previous research with adults (Jones et al., 2001; Wiers et al., 2002), it is noteworthy that these effects are already so apparent in adolescent drinkers who do not have a long history of alcohol (ab)use and alcohol-related problems. These findings seem to be in line with neurobiological models of addiction (e.g. Robinson \& Berridge, 1993; Di Chiara, 2000), which suggest that the effect of incentive motivation processes might already be important in the early development of addictive behaviors. Furthermore, the fact that both implicit negative and implicit positive associations seem to have an influence on prospective drinking behavior may be different from results previously found with bipolar measures (Wiers et al., 2002; 2005), and could indicate that measuring alcoholrelated cognitions in a unipolar fashion is a good idea (cf. Houben \& Wiers, 2006).

However, given a number of limitations, these results should be interpreted with some caution. First, it is complicated to come to any firm conclusions about the relative role of grade (or age) and drinking status, given that these variables are naturally confounded. A second limitation in this study might be that a new measure was used which was based on the IAT. Currently, some authors question the validity of the IAT (e.g. Rothermund \& Wentura, 2004; De Houwer, 2001). It has been proposed that participants performing the IAT may recode the stimuli based on other features than the associations the IAT intends to measure. Participants may sometimes select one 'yes'- or 'figure'-category and one 'no'- or '(back-)ground'-category based on salience. This is referred to as the so-called figure ground asymmetry (Rothermund \& Wentura, 2004). The figure ground asymmetry could play a role in tasks such as the unipolar ST- IAT. However, research indicated that this figure ground asymmetry cannot fully explain all results that were found with unipolar alcohol-IATs similar to the ones used here (Houben \& Wiers, 2006). Third, some of the measures that were used showed a somewhat lower reliability than expected. The internal consistencies of the explicit arousal and sedation expectancy scales were somewhat lower than the positive and 
negative expectancy scales. This could be due to the relative difficulty in finding alcohol expectancy words that are high on arousal or sedation, but are neutral on the valence dimension. Consequently, on average the arousal and sedation expectancy scale were high on arousal or sedation and neutral on the valence dimension, but contained arousal and sedation items that were slightly more positive or negative than neutral. Recently, it has been suggested that alcohol cognition research should consider mapping alcohol-related cognitions along the lines of the combined categories of positive arousal and positive sedation because pure arousal or sedation items which are neutral on valence seem hard to find (cf. Lang, 1995). Additionally, the internal consistency of the unipolar ST-IAT seems to be lower than the previously reported reliability of the bipolar alcohol-IAT (Wiers et al., 2002; Wiers et al., 2005). However, the reported internal consistency of unipolar measures seem to be comparable or better than most other implicit measures (see Houben \& Wiers, 2006)

Although these limitations must be taken into consideration when interpreting the results, this study does provide additional information for the role of alcohol-related cognitions in the early development of drinking alcohol. For several reasons these insights are useful for developing new prevention and intervention methods. First, assessing implicit next to explicit alcohol-related cognitions is important because existing interventions may differentially influence implicit and explicit cognitions (Wiers et al., 2005; cf. Teachman \& Woody, 2003). Second, besides focusing on explicit alcohol-related cognition new interventions could target implicit alcohol-related as well. For instance, alcohol intervention and prevention could focus more on minimizing the influence of hazardous implicit alcohol associations (such as arousal associations). It has been proposed that minimizing the influence of implicit associations that enhance drinking could be obtained by alteration of these fast implicit automatic reactions and/or strengthening of more explicit controlled reactions (Wiers et al., 2004).

Summarizing, both explicit and implicit alcohol-related cognitions seem to influence drinking behavior in young adolescents. The role of implicit alcohol-related cognitions even may be more important than previously assumed. Moreover, interventions may have a differential influence on implicit and explicit cognitions. Therefore, it seems essential that implicit alcohol-related cognitions are taken into account when prevention and intervention methods are being developed. This can be achieved by either the alteration of these fast implicit automatic reactions or strengthening the more explicit controlled reactions. Future research has to indicate if these methods can be included as proven effective components in prevention and intervention programs. 


\section{APPENDIX}

\section{ST-IAT stimuli}

(translated from Dutch)

\begin{tabular}{|c|c|}
\hline & \\
\hline positive (label) & neutral (label) \\
\hline happy & daily \\
\hline sociable & steep \\
\hline nice & related \\
\hline pleasant & wide \\
\hline likeable & usual \\
\hline cosy & flat \\
\hline Negative stimuli & Neutral stimuli \\
\hline negative (label) & neutral (label) \\
\hline unhappy & massive \\
\hline lonely & normal \\
\hline moody & totally \\
\hline sad & recent \\
\hline rude & extensive \\
\hline gloomy & square \\
\hline Arousal stimuli & Neutral stimuli \\
\hline active (label) & neutral (label) \\
\hline talkative & constant \\
\hline jovial & historical \\
\hline restless & oval \\
\hline alert & central \\
\hline unrestrained & blue \\
\hline rambunctious & digital \\
\hline Sedation stimuli & Neutral stimuli \\
\hline passive (label) & neutral (label) \\
\hline silent & direct \\
\hline listless & common \\
\hline sleepy & different \\
\hline quiet & compact \\
\hline relaxed & attached \\
\hline calm & brown \\
\hline
\end{tabular}





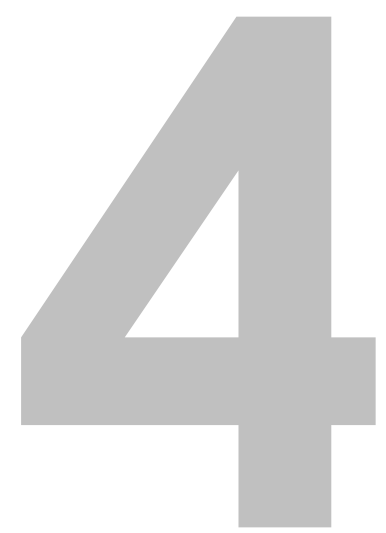

Apples and oranges?

Comparing indirect measures of alcohol-related cognition predicting alcohol use in at-risk adolescents

Published as: Thush, C., Wiers, R.W., Ames,S. L., Grenard/J. L., Sussman, S., \& Stacy, A.W. (2007). Apples and oranges? Comparing indirect measures of alcohol-related cognition predicting alcohol use in at-risk adolescents. Psychology of Addictive Behaviors, 21, 587 -591. 


\section{ABSTRACT}

Recently, there has been increased interest in the role of implicit cognitive processes in the development of addictive behaviors. This study compared three indirect measures of alcohol-related cognitions in the prospective prediction of alcohol use in at-risk adolescents. Implicit alcohol-related cognitions were assessed in 88 Dutch at-risk adolescents ranging in age from 14 to 20 (51 males) with varieties of word association tasks, Implicit Association Tests (IATs) and Extrinsic Affective Simon Tasks (EASTs) adapted for alcohol use. Alcohol use and alcohol-related problems were measured at baseline and after one month with self-report questionnaires. Results showed that the indirect measures predicted unique variance in prospective alcohol use after controlling for the direct measure of alcohol-related cognitions and background variables. The results indicated that the word association tasks were the best indirect measure of alcoholrelated cognitions. These indirect measures appear to assess cognitive motivational processes that affect behavior in ways not reflected by direct measures of alcoholrelated cognitions. 
Dual process models predict that both explicit and implicit cognitive processes influence behavior (e.g. Fazio \& Towles-Schwen, 1999; Strack \& Deutsch, 2004). Implicit cognitive processes represent more automatic underlying motivational processes while explicit cognitions are related to slower deliberate thought processes that may inhibit more automatic, impulsive thinking and behavior (Greenwald \& Banaji, 1995; Kahneman, 2003). Early experiences with alcohol could result in alcohol-related cues (e.g. presence of alcohol), outcomes (e.g. excitement), and behaviors (e.g. drinking) to become associated in memory. These associations may become strengthened over time and guide behavior relatively automatically (Stacy, 1997).

Implicit alcohol-related cognitions have been shown to predict unique variance in current and prospective alcohol use after controlling for explicit alcohol-related cognitions (e.g. Jajodia \& Earleywine, 2003; Palfai \& Wood, 2001; Stacy, 1997; Thush \& Wiers, 2007; Wiers, van Woerden, Smulders, \& De Jong, 2002). Implicit cognitions can be measured with various indirect tasks derived from different research paradigms. Throughout this article we use the terms implicit and explicit cognitive processes or cognitions when referring to the processes evaluated and we use the terms direct or indirect measures when referring to the assessment procedures. Various indirect word association tasks have been derived from basic memory research and found to predict substance use among college students (e.g. Kelly, Masterman, \& Marlatt, 2005; Palfai \& Wood, 2001; Stacy, 1995), community samples (e.g. Stacy \& Newcomb, 1998), drug offenders (e.g. Ames, Zogg, \& Stacy, 2002), and at-risk youth (e.g. Ames, Sussman, Dent, \& Stacy, 2005). In addition, various reaction time paradigms have been adapted to assess automatic alcohol-related cognitions. Among the most commonly researched are variants of the Implicit Association Test (IAT; Greenwald, McGhee, \& Schwartz, 1998) and the Extrinsic Affective Simon Task (EAST; De Houwer, 2003). Both have been found to predict alcohol use and alcohol-related problems among college students (e.g. IAT: Jajodia \& Earleywine, 2003; Wiers et al, 2002; EAST: De Houwer, Crombez, Koster, \& De Beul, 2004; De Jong, Wiers, van den Braak, \& Huijding, 2007). For example, with the use of the IAT, Wiers et al. (2002) found that heavy drinkers associated alcohol more strongly with arousal than with sedation, whereas light drinkers did not, and arousal associations predicted prospective alcohol use. To our knowledge, however, these indirect measures have not been directly compared in the prediction of alcohol use within one study among at-risk youth.

Therefore, this study compared these three indirect measures of alcohol-related cognitions in the prediction of prospective alcohol use in at-risk adolescents while controlling for a variety of other variables. Determining the predictive value of these measures can help to shed some light on which measures are best in predicting future alcohol use among at-risk adolescents. This could benefit our understanding of underlying processes in the development of addictive behaviors and the program effectiveness of targeted interventions among at-risk youth. It was hypothesized that the indirect measures would predict a unique part of the variance in adolescent drinking while 
controlling for confounding and demographic variables (impulsivity, sensation seeking, explicit alcohol-related cognitions, gender, age and school attended). These possible effects were investigated in an exploratory manner without specific hypotheses.

\section{METHOD}

\section{Participants}

A total of 88 Dutch adolescents $(51$ male) in the age range of 14 to 20 (mean age $=$ $16.34, S D=1.34$ ) were recruited from four low level vocation schools. Out of the 88 participants $68(77.27 \%$ ) indicated having one or more binge drinking episodes (5 or more Dutch standard alcoholic drinks on one occasion) in the last 2 weeks.

\section{Measures}

\section{Alcohol Use}

Alcohol use was assessed with a Dutch version of the alcohol use questionnaires as described in Ames et al. (2007). Self-report questionnaires have been proven to be reliable and valid if participant sobriety and confidentiality of data are assured (Sobell \& Sobell, 1990). Both requirements were fulfilled in this study.

\section{Alcohol-Related Problems}

An index of alcohol-related problems was assessed using an 18-item version of the Rutgers Alcohol Problems Index (RAPI; White \& Labouvie, 1989). Participants were asked to indicate on a 5-point Likert scale how many times they experienced certain problems within the last months because of their alcohol use. The items were summed to create an index of alcohol-related problems (Cronbach's alpha $=.87$ ).

\section{Indirect Measures of Alcohol-Related Cognitions}

WORD ASSOCIATION MEASURES. Three types of associative memory tasks were used in which participants were presented with written verbal cues and were instructed to write down the first word it made them think of. In the cue-behavior association task participants responded to 24 Dutch homographs (ambiguous words) including 6 words related to alcohol (an English example is 'pitcher'; Stacy, 1995; Stacy, 1997). The outcome-behavior association task consisted of 13 Dutch affective outcomes of which 6 were positive anticipated consequences of alcohol use (e.g. 'feeling relaxed'; Stacy, 1995; Stacy, 1997). The compound cues were 9 Dutch phrases which consisted of a location and a affective outcome out of which 6 consisted of a high-risk global situation item and a high-risk affective outcome item (e.g. 'friend's house, feeling good'; Stacy, Galaif, Sussman, \& Dent, 1996; Sussman, Stacy, Ames, \& Freedman, 1998). The 


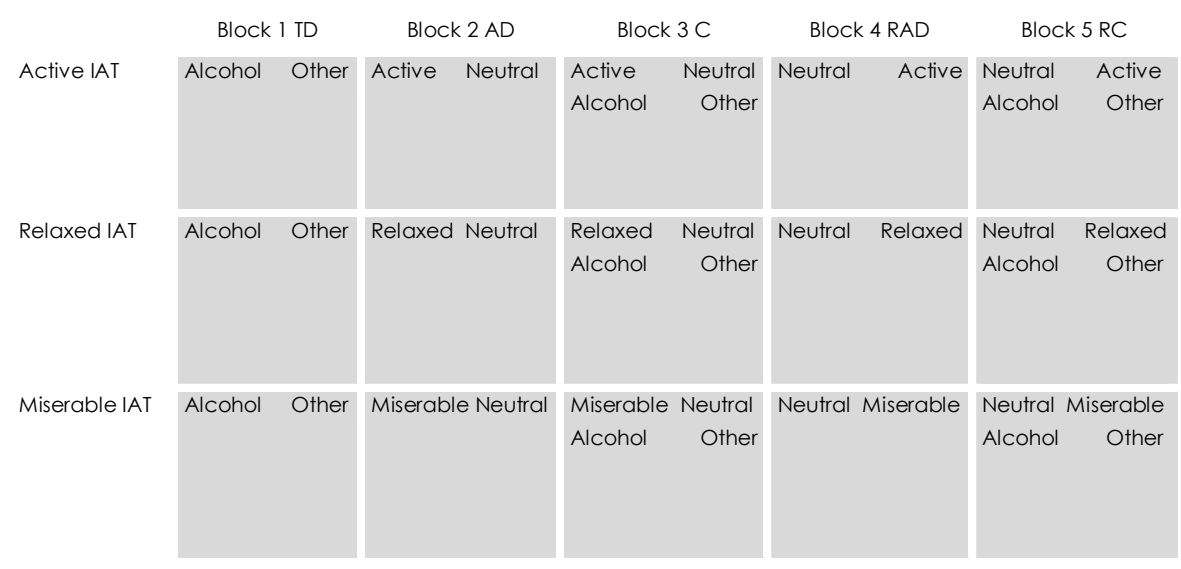

Figure 1. Schematic overview of the block sequence in the three Implicit Association Tests. TD = Target Discrimination, $A D=$ Attribute Discrimination, $C=$ Combination, $R A D=$ Reversed Attribute Discrimination, $R C=$ Reversed Combination.

responses to each cue were coded as being related to alcohol by two independent judges (Kappa's ranging from .72 to .82). A final consensus coding was mediated by a third judge. A mean score was calculated across the alcohol-related cues to provide an indication of the activation of alcohol-related associations to the verbal cues. The scores were combined to form a single composite word association index.

IMPLICIT ASSOCIATION TESTS. In the IAT, participants categorize four categories of stimuli as quickly as possible while only using a left or right response key (see Greenwald et al., 1998). Since prior studies have shown that people can be ambivalent toward alcohol (Houben \& Wiers, 2006), we decided to use three unipolar IATs to obtain the association between alcohol and a single attribute (see Figure 1, for stimuli see Appen$\operatorname{dix}$ A and B). One IAT assessed the association between 'active' positive arousal words (e.g. excited and energetic) versus 'neutral' words (e.g. historical and digital) with photos of objects related to alcohol (e.g. a beer bottle) or objects not related to alcohol (e.g. a ketchup bottle). Another IAT assessed the association between 'relaxed' positive sedation words (e.g. chill and calm) versus neutral words with objects related or not related to alcohol. Lastly, one IAT assessed the association between 'miserable' negative words (e.g. sad and nauseous) versus neutral words with objects related or not related to alcohol. These attribute categories were chosen because they represent the three main categories of alcohol expectancies (Goldman \& Darkes, 2004). The D-2SD penalty score for practice and test was chosen as the main reaction time measure (see Greenwald, Nosek, \& Banaji, 2003)

EXTRINSIC AFFECTIVE SIMON TASKS. In the EAST, participants categorize attribute words in terms of the attribute categories as quickly as possible by using two response keys. These response keys acquire an affective meaning. Subsequently, participants 


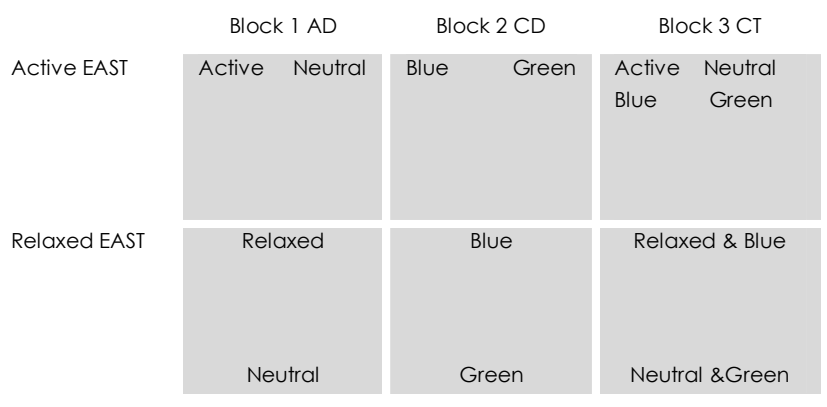

Figure 2. Schematic overview of the block sequence in the two Extrinsic Affective Simon Tasks. $A D=$ Attribute Discrimination, $\mathrm{CD}=$ Color Discrimination, $\mathrm{CT}=$ Combination Test.

categorized colored target words as quickly as possible with respect to their color categories and ignoring their meaning by using the same response buttons. This way, one can assess whether the compatible target words (e.g. when the color of the target word is on the same response key as the associated meaning) will be classified faster than the incompatible target words (e.g. when the color of the target word is on a different response key as the associated meaning) (see De Houwer, 2003). In this study, two unipolar versions of the EAST were used; one EAST assessed the association between positive arousal words versus neutral words with different substances (e.g. beer and marihuana), the other EAST assessed the association between positive sedation words versus neutral words with different substances (see Figure 2). The same attribute words were used as in the two similar IATs (see Appendix C). The EAST scores are obtained by calculating the difference in response latency between the incompatible and compatible target words.

Detailed instructions on the indirect measures (and other measures) are available from Carolien Thush on request.

\section{Other measures}

DIRECT MEASURE OF ALCOHOL-RELATED COGNITIONS. The direct measure of alcohol-related cognitions included 18 items representing an explicit version of the IAT attribute words (as in Wiers et al., 2002). Participants indicated on a 6-point Likert scale the extent to which they (dis)agreed with an item consisting of a statement on drinking alcohol (e.g. 'drinking alcohol makes me feel energetic') The questionnaire consisted of three scales: a positive-arousal, a positive-sedation and a negative outcome scale (Cronbach's alphas ranging from .73 to .84)

IMPULSIVITY AND SENSATION SEEKING SCALE. Impulsivity and sensation seeking were assessed with the 18 items from the Zuckerman-Kuhlman Personality Questionnaire (Zuckerman, Kuhlman, Thornquist, \& Kiers, 1991). Participants were asked to indicate 
whether they thought a statement describing them was true or false. For the two subscales a continuous sum score was calculated (Cronbach's alpha respectively .67 and .65).

\section{Procedure}

After obtaining active consent from both the participants and parents, participants were tested in groups of four at school in a separate test room during school time. The word association tasks were administered first, to ensure that the free associative nature of the task would not be affected by other assessments. Subsequently, the EAST and the IAT were administered on a laptop with a separate response device. The order of tasks within each reaction time paradigm was partially counterbalanced. Next, the participants filled out the direct measure of alcohol related-cognitions. The alcohol use questionnaire was administered last, in order to avoid any interference between having to report ones alcohol use and the measures of implicit and explicit alcohol-related cognitions. The total testing sessions took approximately 90 minutes for each participant. One month later, the participants were asked to fill out the alcohol use and alcohol-related problems questionnaire. All 88 participants were present at follow-up.

\section{Data reduction}

In order to obtain a normally distributed dependent variable and to reduce the chances of a Type I error by multiple testing, we computed a log transformed standardized alcohol use index score. First, $z$ - scores were calculated for eight correlating outcome measures, namely number of times alcohol used in lifetime, number of times alcohol used in the last past month, the number of standard drinks on a weekend day, the number of standard drinks on a week day, number of times drunk in the last year, frequency of binges per two week, the number of binges in the last week, and the sum score on the RAPI. Subsequently, the alcohol use index score was computed by log transforming the mean of these eight z-scores to obtain a normally distributed dependent variable.

\section{RESULTS}

\section{Outliers}

Two participants reported never to have consumed alcohol were eliminated from further analyses. In addition, 5 participants were excluded from further analyses because they exceeded the mean error scores in a reaction time task with more than 3 standard deviations (see Greenwald et al., 2003). The analytic sample was 81. 
Table 1

Pearson Correlation for Explicit and Implicit Cognition and Alcohol Use

\begin{tabular}{|c|c|c|c|c|c|c|c|c|c|c|}
\hline & 1 & 2 & 3 & 4 & 5 & 6 & 7 & 8 & 9 & 10 \\
\hline 1. Expl Active & - & & & & & & & & & \\
\hline 2. Expl Relaxed & .01 & - & & & & & & & & \\
\hline 3. Expl Miserable & -.11 & $-.45^{* *}$ & - & & & & & & & \\
\hline 4. IAT Active & -.12 & -.02 & .13 & - & & & & & & \\
\hline 5. IAT Relaxed & -.03 & .13 & -.06 & $.21 \#$ & - & & & & & \\
\hline 6. IAT Miserable & -.05 & -.09 & .06 & $.27^{*}$ & -.11 & - & & & & \\
\hline 7. EAST Active & .05 & .12 & .04 & $-.19 \#$ & -.15 & -.17 & - & & & \\
\hline 8. EAST Relaxed & -.08 & $.30 * *$ & $-.23^{*}$ & .16 & -.04 & -.12 & -.08 & - & & \\
\hline 9. Word Associations Index & -.04 & $.40^{* *}$ & $-.30^{* *}$ & .10 & .11 & -.10 & -.02 & $.22^{*}$ & - & \\
\hline 10. Alcohol Use Index & $.22 \#$ & $.44^{* *}$ & $-.46^{* *}$ & .01 & -.02 & $-.23^{*}$ & -.01 & $.26^{*}$ & $.43^{* *}$ & - \\
\hline
\end{tabular}

Note. Expl Active = explicit positive-arousal alcohol cognitions; Expl Relaxed $=$ explicit positive-sedation alcohol cognitions; Expl Miserable = explicit negative alcohol cognitions; IAT Active = D-2SD score for the positive-arousal IAT; IAT Relaxed = D-2SD score for the positive-sedation IAT; IAT Miserable = D-2SD score for the negative IAT; EAST Active = mean reaction time difference score for positive-arousal EAST; EAST Relaxed = mean reaction time difference score for positive-sedation EAST; Word Associations Index = mean score of alcohol cues, alcohol outcomes and compound cues; Alcohol Use Index = log transformed standardized sum score of eight outcome variables. \# $p \leq .10$, two tailed $* p<.05$, two-tailed. ${ }^{* *} p<.01$, two-tailed.

\section{Multiple Regression Analyses}

Multiple regression models for the alcohol use index were evaluated based on the results of the bivariate analyses; only the variables that were (borderline) significantly correlated with the alcohol use index were included in the multiple regression (see Table 1). By using this procedure an optimal trade-off between statistical power (including all variables reduces power) and completeness (not leaving out important variables) was ensured. A setwise hierarchical procedure was used for the multiple regression analyses (Cohen \& Cohen, 1983). In Step 1, gender and school attended were entered into the regression equation as background variables. In Step 2 sensation seeking and impulsivity were added to the regression equation. In Step 3 the three direct measures of alcohol-related cognition were added to the model. In Step 4 three indirect measures of alcohol-related cognition were added to the regression model. The hierarchical regression model revealed that overall as a set the indirect measures added significantly to the prediction of prospective drinking $\left(\Delta R^{2}=.06, p<.05\right)$ above and beyond the background variables and direct measures (see Table 2). Overall, the full model explained $54 \%$ of the variance in the alcohol use index $\left(R^{2}\right.$ adjusted $=.46, F$ $(12,67)=6.56, p<.001)$. A trimmed model was obtained by removing all variables that were not significant from the regression equation. The final trimmed model revealed that the word associations $(\beta=.30, p<.01)$ predicted the alcohol use index after one month adjusting for other predictors that were also significant including school $\beta=.30$, $p<.01$ ), impulsivity $(\beta=.21, p<.05)$, explicit positive-arousal alcohol-related cognitions ( $\beta$ $=.20, p<.05)$ and explicit negative alcohol-related cognitions $(\beta=-.40, p<.001)$. 
Table 2

Summary of Multiple Regression Analysis for Variables Predicting Alcohol Use After One Month $(\mathrm{N}=81)$

\begin{tabular}{|c|c|c|c|c|c|}
\hline \multirow[b]{2}{*}{ Variable } & \multicolumn{2}{|c|}{ Cumulative } & \multicolumn{3}{|c|}{ Simultaneous } \\
\hline & $\mathrm{R}^{2}$ & $\Delta R^{2}$ & B & SE B & $\beta$ \\
\hline Gender & & & 0.13 & 0.15 & .09 \\
\hline School 1 & & & 0.10 & .31 & .03 \\
\hline School 3 & & & 0.37 & 0.18 & $.21^{*}$ \\
\hline School 4 & .12 & & -0.14 & 0.16 & -.09 \\
\hline Sensation Seeking & & & 0.28 & 0.37 & .08 \\
\hline Impulsivity & .17 & .06 & 0.55 & 0.31 & .19 \\
\hline Expl Active & & & 0.17 & 0.07 & $.22^{*}$ \\
\hline Expl Relaxed & & & 0.11 & 0.09 & .14 \\
\hline Expl Miserable & .48 & $.31^{* *}$ & -0.26 & 0.08 & $-.32^{* *}$ \\
\hline IAT Miserable & & & -0.17 & 0.11 & -.14 \\
\hline EAST Relaxed & & & 0.00 & 0.00 & .08 \\
\hline Word Association Index & .54 & $.06^{*}$ & 1.70 & 0.82 & $.20^{*}$ \\
\hline
\end{tabular}

Note. $R^{2}$ and $\Delta R^{2}$ are from hierarchical models in which preceding effects were entered first; the $B, S E B$ and $\beta$ are from a simultaneous model. Sensation Seeking = summed mean sensation seeking score; Impulsivity = summed mean impulsivity score ; Expl Active = explicit positive-arousal alcohol cognitions; Expl Relaxed $=$ explicit positive-sedation alcohol cognitions; Expl Miserable = explicit negative alcohol cognitions; IAT Miserable = D-2SD score for the negative IAT; EAST relaxed = mean reaction time difference score for the positive sedation EAST; Word Associations Index = mean score of alcohol cues, alcohol outcomes and compound cues. ${ }^{*} p<.05{ }^{* *} p<.01$

Overall, the trimmed model explained $49 \%$ of the variance $\left(\mathrm{R}^{2}\right.$ adjusted $=.44, F(7,73)=$ $10.08, p<.0011$.

\section{DISCUSSION}

This study compared three indirect measures of alcohol-related cognitions that were used to predict prospective alcohol use in at-risk adolescents. The multiple regression analysis showed that the indirect measures predicted unique variance in prospective alcohol use after controlling for explicit alcohol-related cognitions and background variables. This is in line with previous research on implicit and explicit alcohol-related cognitive processes in young adults and adolescents (e.g. Palfai \& Wood, 2001; Stacy, 1997; Thush \& Wiers, 2007; Wiers et al., 2002). Additionally, the trimmed model showed that the word association tests were the strongest predictor among the group of indirect measures of alcohol use. Similar findings were reported in a comparison of word association, IAT, and EAST in the prediction of marijuana use among at-risk youth in the U.S. (Ames et al., 2007). Thus, the same pattern of result arises across different countries, languages and substances.

One possible explanation for the current pattern of results is that the IAT measures relative associations between predefined categories. In contrast, word association allows for free competition among associates to be generated in response to a variety of cues. Because these word association tasks do not impose categorical constraints on 
the individual, they increase the likelihood of tapping into individual differences in underlying motivational associative structures. On the one hand, the reaction time measures seem to be limited in the sense that they place categorical constraints on the activation of associative structures, while on the other hand, these categorical constraints could be helpful when specific hypotheses about the content of alcohol-related associations are being assessed. In sum, comparing these tasks is like comparing apples and oranges; both types of indirect measures (word association tasks and reaction time measures) have their own unique strengths for which they can be used in addiction research.

Given several limitations, the results presented in this study should be interpreted with some caution. First, although we screened for schools with a high proportion of atrisk adolescents, we did not use a probability sampling strategy at an individual level and therefore we might not be able to generalize these results to other at-risk adolescent populations. Second, we did not have the statistical power to adequately look at possible interactions with other background variables such as gender. It could be that for specific subgroups of at-risk adolescents the results would have been different. Third, since the word association tests, EAST and IAT were performed in a fixed order, it is possible that order effects could have played a role in the current results. However, this fixed sequence was chosen as the most optimal procedure to minimize method-related variance in a study focusing on individual differences (cf. Asendorpf, Banse, \& Mücke, 2002). Fourth, although measuring implicit associations in a unipolar fashion may have a better construct validity given that people can be ambivalent towards alcohol (Houben \& Wiers, 2006), measuring associations in a bipolar fashion could provide better power since it allows a more natural competition between several associations which exist side by side to each other (e.g. the competition between a positive and a negative association with alcohol). This could partly explain why both the IAT and EAST were not more predictive and were sometimes significant correlates only in the bivariate analysis. Finally, it is possible that assessing too many drugs in one unipolar EAST is problematic, minimizing activation of associative structures and thus the EAST effects in this study.

In sum, both explicit and implicit alcohol-related cognitions seem to predict drinking behavior at-risk adolescents. Overall, the indirect measures added significantly to the prediction of prospective drinking while controlling for background variables and direct measures. More specifically, word associations predicted drinking after one month adjusting for school, impulsivity, explicit positive-arousal and negative alcohol cognitions. Although the reaction time measures place categorical constraints on the activation of an associative network, these categorical constraints could still be helpful when specific hypotheses about are being assessed. Thus, depending on the nature of the hypotheses to be assessed both word association tasks and reaction time measures have their own unique strengths for which they can be used in addiction research. 
APPENDIX A

\section{IAT Picture Stimuli}

Alcohol stimuli

Label: alcohol photo
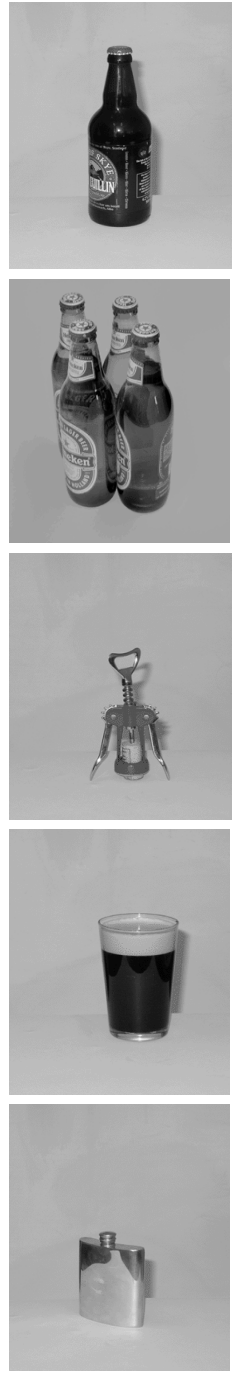

Neutral stimuli

Label: other photo
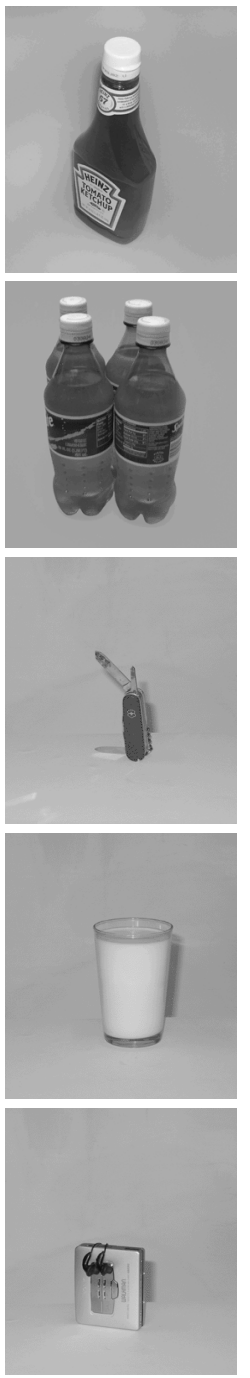


\section{APPENDIX B}

\section{IAT Word Stimuli}

(Translated from Dutch)

Positive Arousal stimuli
(active word)
excited
energetic
busy
lively
wild

Neutral stimuli

(neutral word)

historical

apart

steep

wide

compact

Positive Sedation stimuli

Neutral stimuli

(relaxed word)

(neutral word)

relaxed

normal

calm

blue

chill

flat

tranquil

central

comfortable

digital

Negative stimuli

Neutral stimuli

(miserable word)

(neutral word)

sad

daily

pain

square

sick

narrow

nauseous

common

miserable

totally 


\section{APPENDIX C}

\section{EAST Stimuli}

(Translated from Dutch)

Substance-Related Words

$\begin{array}{llll}\text { Beer } & \text { Tobacco } & \text { Marijuana } & \text { XTC } \\ \text { Liquor } & \text { Cigarette } & \text { Weed } & \text { speed } \\ \text { vodka } & \text { smoke } & \text { cannabis } & \text { cocaine } \\ \text { Cocktail } & \text { lighter } & \text { Stuff } & \text { crack }\end{array}$

Positive Arousal stimuli

Neutral stimuli

(active word)

(neutral word)

excited

historical

energetic

apart

busy

steep

lively

wide

wild

compact

Positive Sedation stimuli

Neutral stimuli

(relaxed word)

(neutral word)

relaxed

normal

calm

blue

chill

flat

tranquil

central

comfortable

digital 



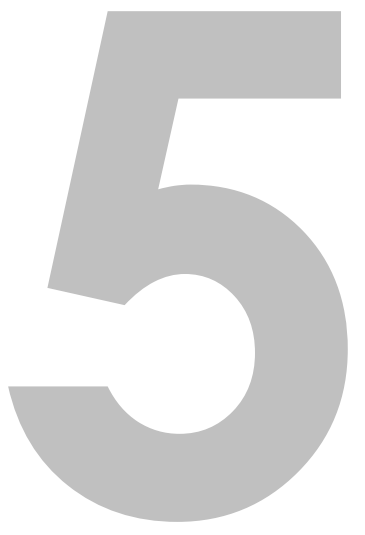

Interactions between implicit and explicit cognition and working memory capacity in the prediction of alcohol use in at-risk adolescents

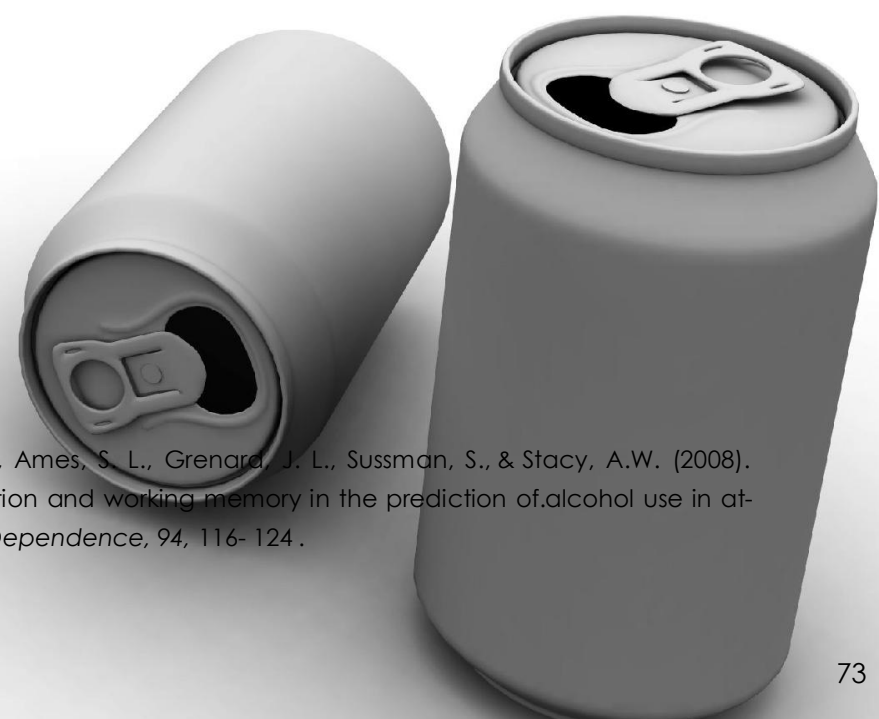




\section{ABSTRACT}

Dual process models of addiction suggest that the influence of alcohol-related cognition might be dependent on the level of executive functioning. This study investigated if the interaction between implicit and explicit alcohol-related cognitions and working memory capacity predicted alcohol use after one month in at-risk youth. Implicit and explicit alcohol-related cognitions were assessed in 88 Dutch at-risk adolescents ranging in age from 14 to 20 (51 males) with an adapted version of the Implicit Association Test (IAT) and an expectancy questionnaire. Working memory capacity was assessed using the computer-based version of the Self-Ordered Pointing Task (SOPT). Alcohol use and alcohol-related problems were measured at baseline and after one month with self-report questionnaires. The hierarchical regression analysis showed that both the interaction between implicit positive-arousal cognitions and working memory capacity and the interaction between explicit positive-arousal cognitions and working memory capacity predicted unique variance in alcohol use after one month. Implicit positivearousal cognitions predicted alcohol use after one month more strongly in students with lower levels of working memory capacity, whereas explicit positive-arousal cognitions predicted one-month follow-up alcohol use more strongly in students with higher levels of working memory capacity. This could imply that different intervention methods could be effective for different subgroups of at-risk youth. 
Several dual process models predict that both more reflective explicit and more impulsive implicit cognitive processes influence behavior (e.g. Fazio \& Towles-Schwen, 1999; Kahneman, 2003; Strack \& Deutsch, 2004). Implicit cognitions represent more automatic underlying motivational processes, whereas explicit cognitions are related to more deliberate thought processes (Greenwald \& Banaji, 1995; Kahneman, 2003). Recently, there has been increased interest in the role of implicit cognitions in the development of addictive behaviors. These implicit alcohol-related cognitions represent individual differences in memory associations between alcohol-related cues (e.g. presence of alcohol), outcomes (e.g. excitement), and behaviors (e.g. drinking). These associations if strengthened over time become motivationally significant and guide behavior relatively automatically (Stacy, 1997). The mesolimbic dopamine reward system has been associated with these relatively automatic motivational processes, which are believed to play an important role in the development of addictive behavior (e.g. Bechara, 2005; Kalivas \& Volkow, 2005). Implicit cognitions have been shown to predict unique variance in current and prospective alcohol use after controlling for explicit cognitions (e.g. Jajodia \& Earleywine, 2003; Palfai \& Wood, 2001; Stacy, 1997; Thush \& Wiers, 2007; Wiers, Van Woerden, Smulders, \& De Jong, 2002).

A second assumption of several dual process models is that the influence of implicit processes on subsequent behavior is moderated by explicit processes if motivation and the opportunity to do so are present (e.g. Fazio \& Towles-Schwen, 1999; Kahneman, 2003; Strack \& Deutsch, 2004). Indeed, neurobiological addiction research has shown that the prefrontal cortex and associated areas are involved in more reflective decision making and in the moderation of impulses (e.g. Bechara, 2005; Kalivas \& Volkow, 2005; Wilson, Sayette \& Fiez, 2004). Dual process models of addiction specifically predict that the influence of implicit appetitive cognitions on subsequent addictive behavior might be dependent on the level of executive functioning (e.g. Stacy, Ames \& Knowlton, 2004; Wiers et al, 2007).

Executive functions can be described as a set of cognitive skills relevant to goaldirected behavior involving different abilities such as shifting, updating and inhibition (Miyake et al., 2000). Working memory capacity has been proposed to be a central construct that possibly binds these different but related executive functions (Kane \& Engle, 2002). The relationship between executive functions and alcohol use has been shown to be bidirectional. Poorer executive functioning can be considered a risk factor for developing addictive behaviors such as drinking alcohol (e.g. Finn \& Hall, 2004; Peterson, Finn \& Pihl, 1992; Tapert, Baratta, Abrantes \& Brown, 2002). Additionally, alcohol abuse has been shown to negatively affect the maturation of brain regions (e.g. DeBellis et al., 2000), to impair neuropsychological functioning (e.g. Brown, Tapert, Granholm \& Dellis, 2000) and to alter processing on executive functioning tasks (Tapert et al., 2004). During adolescence, when executive functions and associated brain regions are still developing, alcohol induced damage in the prefrontal cortex can lead to inhibitory and attentional control problems which, in turn, may influence continued 
alcohol use (Crews et al., 2000; Wiers et al, 2007). This bidirectional nature might be more apparent in at-risk adolescents. It is also possible that these at-risk adolescents may start with poorer executive functions and begin drinking at earlier ages, which, in turn, further interferes with their ability to control their drinking behavior.

Prior research has suggested that the influence of implicit automatic processes, on other behaviors, indeed, is moderated by executive control (e.g. Feldman-Barrett, Tugade \& Engle, 2004; Payne, 2005), as has also been proposed for addictive behaviors (e.g. Stacy et al., 2004; Wiers et al, 2007). Grenard et al. (in press) evaluated the interaction between working memory capacity and spontaneous memory associations assessed with word association tasks (an alternative indirect assessment of implicit cognitive processes) among at-risk youth and found evidence that drug-relevant associations were stronger predictors of alcohol and cigarette use among those with lower working memory capacity than among those with higher working memory capacity. Finn and Hall (2004) proposed that two mechanisms might be responsible for the moderating influence of executive functioning on the implicit processes-behavior relationship. First, low activating capacity of working memory makes it difficult to shift attention away from highly activated stimuli to stimuli that are less salient. Second, short term positive associations with behavior tend to be highly activated (salient), whereas the long term negative associations with behavior are usually weakly activated. Consequently, in high-risk situations, such as being at a party where alcohol is readily available, an individual needs to be able to switch to less immediately salient goals and attend to them - such as the intention to not drink large amounts of alcohol or binge drink - while distracting salient information in the current high-risk situation is more automatically activated (e.g., the urge to feel intoxicated or to give in to peer pressure). This relationship between executive functioning and behavior suggests that adolescents who are less able to actively manage less salient but adaptive goals when faced with distracting information are more likely to let their behavior be influenced by distracting salient information that is triggered in the current situation (e.g. Stacy et al., 2004; Wiers et al, 2007).

Conversely, research suggests that explicit positive expectancies might be moderated by executive functioning in the opposite direction. In addiction research, alcohol expectancies have been shown to be good predictors of concurrent and, to a lesser extent, prospective alcohol use (e.g., Goldman \& Darkes, 2004; Jones, Corbin \& Fromme, 2001; Sher, Wood, Wood \& Raskin, 1996; Stacy, Newcomb \& Bentler, 1991). Tapert, McCarthy, Aarons, Schweinsburg, \& Brown (2003) showed that explicit positive alcohol expectancies predicted alcohol use in substance use disorder adolescents with good verbal skills, but not in substance use disorder adolescents with poor verbal skills. Verbal skills are needed for developing internal language-based reasoning skills (Luria, 1961) and predictive of positive alcohol expectancies (Deckel, Hesselbrock \& Baver, 1995). Verbal skill tasks such as the verbal fluency task are commonly regarded as measuring frontal-lobe processing (Deckel et al., 1995) and verbal executive function- 
ing (Tapert et al., 2003). Tapert et al. (2003) concluded that positive alcohol expectancies require encoding and deep processing in order for them to affect decision making and thus drinking. However, other studies have found that adolescents who show good executive functioning (e.g. good inhibitory neural processing) generally had fewer positive and more negative outcome alcohol expectancies (Anderson, Schweinsburg, Paulus, Brown, \& Tapert, 2005) and that verbal skills sometimes are also negatively predictive of positive expectancies (Deckel et al., 1995). Thus, there are still some questions about whether positive alcohol expectancies might be moderated by executive functioning in the opposite direction than implicit appetitive associations.

Hence, from a dual process perspective we hypothesized that the influence of implicit appetitive associations (both positive-arousal and positive-sedation) on subsequent drinking behavior is stronger in adolescents with low working memory capacity than in adolescents with high working memory capacity. Conversely, the influence of explicit positive-arousal alcohol-related processes on subsequent drinking behavior is hypothesized to be stronger in adolescents with high working memory capacity compared with adolescents with low working memory capacity. The current study investigated these two interactions between alcohol-related cognition and working memory capacity in the prediction of alcohol use after one month in at-risk youth.

\section{METHOD}

\section{Participants}

A total of 88 Dutch adolescents $(51$ male) in the age range of 14 to 20 (mean age = $16.34, S D=1.34$ ) were recruited from four low-level vocational schools (Thush, Wiers, Ames et al., 2007). Based on a Dutch national health survey among high school adolescents, low-level vocational schools showed the highest prevalence of alcohol and drug use as well as behavioral problems. Hence, at the group level, these students are considered to be at risk for developing substance-related problems. Participants selfreported drinking an average of 10.85 Dutch standard alcoholic drinks on a weekend day (SD $=10.30$ ) and an average of 2.72 Dutch standard alcoholic drinks on a weekday $(S D=6.18)$. (A standard alcohol serving in the Netherlands contains somewhat less alcohol than a standard American standard glass: $10 \mathrm{vs.} 14 \mathrm{~g}$ ). Of the 88 participants 68 $(77.27 \%)$ indicated having one or more binge drinking episodes (5 or more Dutch standard alcoholic drinks on one occasion) in the past 2 weeks. 


\section{Measures}

\section{Alcohol Use}

Alcohol use was assessed with a Dutch version of the alcohol use questionnaires as described in Ames et al. (2007). Self-report questionnaires have been proven to be reliable and valid if participant sobriety and confidentiality of data are assured (Sobell \& Sobell, 1990). Both requirements were fulfilled in this study. Participants were asked how many times they used various drugs in the last month and in their lifetime on an 11 item rating scale (ranging from 'never used' to '91-100+ times'). Participants respond to a list of specific drugs (i.e. alcohol, marijuana, ecstasy, etc.) or more general drug categories (i.e. hallucinogens, stimulants, other club drugs, etc.). Additionally, participants indicated how many Dutch standard glasses of alcohol they consumed at the last weekend day and weekday they drank. In addition, they indicated on a 3-point Likert scale how many standard glasses of alcohol they consumed on each day of the past week (ranging from 'no drinks' to 'more than 5 drinks'), and they indicated on a 6point Likert scale on how many occasions they drank five Dutch standard glasses of alcohol or more in the past two weeks (ranging from 'I do not drink' to 'seven times or more'). Lastly, participants indicated on a 5-point Likert scale (ranging from 'I do not drink' to 'very intoxicated') how intoxicated they were the last time they drank in the past 12 months.

\section{Alcohol-Related Problems}

An index of alcohol-related problems was assessed using an adapted version of the Rutgers Alcohol Problems Index (RAPI; White \& Labouvie, 1989). We used the 18-item version of the RAPI which correlated .99 with the original 23-items version (White \& Labouvie, 2000). Participants were asked to indicate on a 5-point Likert scale (ranging from 'never' to 'daily') how many times they experienced certain problems within the last months because of their alcohol use. An example of an item is: "Not able to do your homework or study for a test". The items were summed to create an index of alcohol-related problems (Cronbach's alpha $=.87$ ).

Implicit Association Test

In the IAT, participants categorize stimuli into four categories as quickly as possible while only using a left or right response key (see Greenwald, McGhee \& Banaji, 1998). Since prior studies have shown that people can be ambivalent toward alcohol (Houben \& Wiers, 2006), we decided to use three unipolar IATs to obtain the association between alcohol and a single attribute. One IAT assessed the association between 'active' positive arousal words versus 'neutral' words with photos of objects related to alcohol or objects not related to alcohol. Another IAT assessed the association between 'relaxed' positive sedation words versus neutral words with objects related or not related to alcohol. 


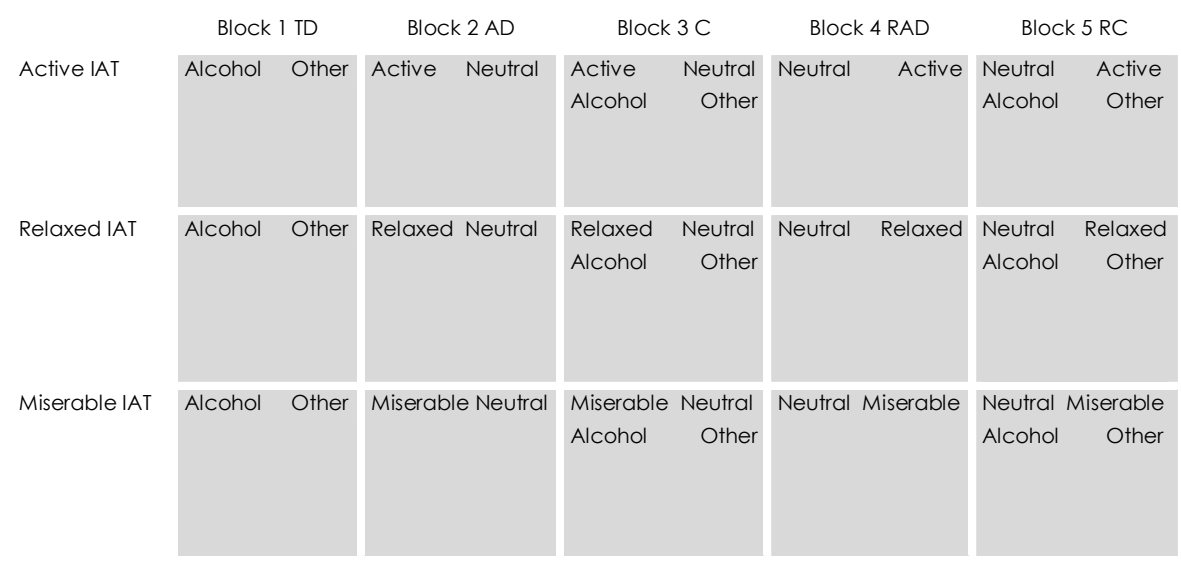

Figure 1. Schematic overview of the block sequence in the three Implicit Association Tests. TD = Target Discrimination, $A D=$ Attribute Discrimination, $C=$ Combination, $R A D=$ Reversed Attribute Discrimination, $R C=$ Reversed Combination.

Lastly, one IAT assessed the association between 'miserable' negative words versus neutral words with objects related or not related to alcohol. These attribute categories were chosen because they represent the three main categories of alcohol expectancies (Goldman \& Darkes, 2004). Stimulus pictures, in addition to stimulus words, were used in order to be able to have a neutral contrast category next to the alcohol target category. The perceptual features of the stimulus pictures were matched by using pictures of objects with approximately the same background, color, size and shape (see Appendix A). The words used (see Appendix B) were matched on number of letters, syllables, familiarity, and on valence and arousal values. The valence and arousal values of stimulus words were matched on group level (positive-arousal, positive-sedation, negative and neutral words) by using student word ratings (for exact procedure see Ames et al., 2007). In this study the three IATs were used in partially balanced order and programmed in ERTS 3.18 (Beringer, 1996). Each attribute discrimination block consisted of 20 trials and each combination block consisted of 40 trials. The D-2SD penalty score for practice and test was chosen as the main reaction time measure (see Greenwald, Nosek \& Banaji, 2003). The internal consistency between practice (10) and test items (30) was acceptable (Cronbach's alpha ranging from .52 to .59) and comparable to most other implicit measures (see Houben \& Wiers, 2006).

\section{Expectancy Questionnaire}

The direct measure of alcohol-related cognitions was an expectancy questionnaire consisting of an 18 unipolar item representing an explicit version of the implicit test words (see Appendix B; as in Wiers et al. 2002). Each item consisted of a statement on 
Table 1

Pearson Correlations for Explicit and Implicit Cognition and Working Memory Capacity

\begin{tabular}{|c|c|c|c|c|c|c|c|}
\hline & 1 & 2 & 3 & 4 & 5 & 6 & 7 \\
\hline 1. Expl. Active & - & & & & & & \\
\hline 2. Expl. Relaxed & .01 & - & & & & & \\
\hline 3. Expl. Miserable & -.11 & $-.45^{* *}$ & - & & & & \\
\hline 4. IAT Active & -.12 & -.02 & .13 & - & & & \\
\hline 5. IAT Relaxed & -.03 & .13 & -.06 & .21 & - & & \\
\hline 6. IAT Miserable & -.05 & -.09 & .06 & $.27^{*}$ & -.11 & - & \\
\hline 7. WMC & .06 & -.08 & .03 & .02 & -.01 & .21 & - \\
\hline
\end{tabular}

Note. Expl Active = explicit positive-arousal alcohol cognitions; Expl Relaxed = explicit positive-sedation alcohol cognitions; Expl Miserable = explicit negative alcohol cognitions; IAT Active = D-2SD score for the positive-arousal IAT; IAT Relaxed = D-2SD score for the positive-sedation IAT; IAT Miserable = D-2SD score for the negative IAT; WMC = working memory capacity expressed in total number of correct responses on three consecutive Self Order Pointing Tasks. ${ }^{*} p<.05$, two-tailed. ${ }^{* *} p<.01$, two-tailed.

drinking alcohol (for example: 'drinking alcohol makes me feel energetic'). Participants indicated the extent to which they (dis)agreed with each item on a 6-point Likert scale. The questionnaire consisted of three scales: a positive-arousal, a positive-sedation and a negative outcome scale. The internal consistency of all three scales was good (Cronbach's alphas ranging from .73 to .84)

\section{Self-Ordered Pointing Task}

The Self-Ordered Pointing Task (SOPT) used in the current study was a computer-based version (Peterson, Pihl, Higgins \& Lee, 2002) of the assessment developed by Petrides and Milner (1982) to measure working memory capacity. Participants were instructed to select pictures from a $3 \times 4$ matrix of 12 pictures. Each time a participant selected one picture, the arrangement of pictures in the matrix changed. The participant had to select a picture on a different location that had not been selected previously. The participant continued to select a different picture on each of 12 screen displays. The task was administered in three repetitions of the task using pictures of concrete items (e.g., a calculator, bus, stopwatch).Each task consisted of 12 trials and thus there were 36 trials in total. The number of correct responses is summed across the three tasks, with higher scores indicative of good working memory capacity. The internal consistency between the number of correct pictures across the three trials was good (Cronbach's alpha $=.74$ )

\section{Procedure}

Participants were recruited through five-minute classroom enrollment talks. After obtaining active consent from both the participants and parents, participants were tested in small groups of four at school in a separate test room during school time. The IAT was administered on a laptop with a separate response device. The order of the IAT was partially counterbalanced. The instructions were given on the computer screen preced- 
ing the task. Throughout the trials of both tasks, participants received feedback in red letters on their screen (incorrect response= 'ERROR', > 3000 ms. = 'TOO SLOW', < 150 ms. = 'TOO FAST'). Next, the computer-based SOPT was administered. The instructions were given verbally by the experimenter. Throughout the trials of the tasks, participants received limited feedback on their screen; participants only received an error message indicating they had to select another picture when they had selected a picture on the same picture location as in the preceding turn. Subsequently, the participants filled out the expectancy questionnaire. The alcohol use questionnaire was administered last, in order to avoid any interference between having to report ones alcohol use and the measures of implicit and explicit alcohol-related cognitions. One month later, the participants were asked to fill out the alcohol use and alcohol-related problems questionnaire. All 88 participants were present at follow-up.

\section{Data Reduction}

In order to obtain a normally distributed dependent index variable and to reduce the chances of a Type I error by multiple testing, we computed a log transformed standardized alcohol use index score in three consecutive steps. First, $z$ - scores were calculated for eight different correlated outcome measures, namely number of times alcohol used in lifetime, number of times alcohol used in the past month, the number of standard drinks on a weekend day, the number of standard drinks on a weekday, number of times drunk in the last year, frequency of binges per two weeks, the number of binges in the last week, and the total sum score on the RAPI. Subsequently, the alcohol use index score was computed by calculating the mean of these eight $z$-scores. Finally, the alcohol use index was log transformed to obtain a normally distributed dependent variable.

\section{RESULTS}

\section{Outliers}

Analyses included only those participants reporting that they had consumed alcohol in their lifetime. Two participants reported that they had never consumed alcohol and were therefore eliminated from further analyses. In addition, 5 participants were excluded from further analysis because they exceeded the mean error scores in the reaction time task by more than 3 standard deviations. The SOPT scores of participants that exceeded the mean by more than 3 standard deviations were recoded to the lowest value after removal of the outliers minus 1 (a method referred to as winsorizing). The analytic sample was 81 . 
Table 2

Summary of Hierarchical Regression Analysis for Variables Predicting Alcohol Use After One Month $(N=81)$

\begin{tabular}{|c|c|c|c|}
\hline Variable & $B$ & SE B & $\beta$ \\
\hline \multicolumn{4}{|l|}{ Stepl } \\
\hline Gender & 0.29 & 0.17 & .19 \\
\hline Age & -0.05 & 0.08 & -.09 \\
\hline School 1 & -0.16 & 0.36 & -.05 \\
\hline School 3 & 0.46 & 0.28 & .27 \\
\hline School 4 & -0.15 & 0.21 & -.10 \\
\hline \multicolumn{4}{|l|}{ Step 2} \\
\hline Gender & 0.39 & 0.17 & $.25^{*}$ \\
\hline Age & -0.05 & 0.08 & -.08 \\
\hline School 1 & -0.03 & 0.36 & -.01 \\
\hline School 3 & 0.40 & 0.27 & $.23^{*}$ \\
\hline School 4 & -0.19 & 0.20 & -.12 \\
\hline Expl. Active & 0.21 & 0.09 & $.27^{*}$ \\
\hline IAT Active & 0.02 & 0.12 & .02 \\
\hline WMC & 0.00 & 0.03 & .02 \\
\hline \multicolumn{4}{|l|}{ Step 3} \\
\hline Gender & 0.38 & 0.17 & $.25^{*}$ \\
\hline Age & -0.06 & 0.08 & -.11 \\
\hline School 1 & -0.14 & 0.35 & -.04 \\
\hline School 3 & 0.31 & 0.27 & .18 \\
\hline School 4 & -0.22 & 0.20 & -.14 \\
\hline Expl. Active & 0.16 & 0.09 & .20 \\
\hline IAT Active & 0.06 & 0.12 & .06 \\
\hline WMC & 0.00 & 0.03 & .01 \\
\hline WMC X Expl. Active & 0.09 & 0.04 & $.28^{*}$ \\
\hline \multicolumn{4}{|l|}{ Step 4} \\
\hline Gender & 0.37 & 0.16 & $.24^{*}$ \\
\hline Age & -0.03 & 0.08 & -.05 \\
\hline School 1 & -0.16 & 0.34 & -.05 \\
\hline School 3 & 0.14 & 0.27 & .08 \\
\hline School 4 & -0.25 & 0.19 & -.16 \\
\hline Expl. Active & 0.12 & 0.09 & .15 \\
\hline IAT Active & 0.02 & 0.12 & .02 \\
\hline WMC & 0.02 & 0.03 & .07 \\
\hline WMC X Expl. Active & 0.09 & 0.04 & $.28^{*}$ \\
\hline WMC X IAT ACtive & -0.09 & 0.04 & $-.25^{*}$ \\
\hline
\end{tabular}

Note. $R^{2}=0.12(p=.07)$ for Step $1 ; \Delta R^{2}=.07(p=.13)$ for Step $2 ; \Delta R^{2}=.06(p<.05)$ for Step $3 ; \Delta R^{2}=.05(p<.05)$ for Step 4. Expl. Active = explicit positive-arousal alcohol expectancies; IAT Active = Implicit Association Test D2SD-score expressing the strength of the positive-arousal association with alcohol; WMC = working memory capacity expressed in total number of correct responses on three consecutive Self Order Pointing Tasks. ${ }^{*} p<.05{ }^{* *} p<.01$ 


\section{Bivariate Analyses}

As is shown in Table 1, the IAT measures and the alcohol expectancy measures were not correlated with each other (all $p>$.20). Furthermore, both the IAT measures and the alcohol expectancy measures were not correlated with the SOPT lexcept for the positive correlation between the negative IAT and SOPT of $r=.21, p=.06$, all $p$-values > .40). Additionally, although prior research has shown that poor working memory has been reliably associated with alcohol problems, the RAPI did not correlate with the SOPT $(r=.07, p=.52)$.

\section{Multiple Regression Analyses}

To assess whether there was a significant interaction between working memory capacity and alcohol-related cognition in the prediction of alcohol use, three hierarchical regression models for alcohol use were evaluated. One model tested the hypothesis that implicit and explicit positive-arousal cognitions interacted with working memory capacity. Another model tested the hypothesis that implicit and explicit positivesedation cognitions interacted with working memory capacity. Lastly, one model tested the hypothesis that implicit and explicit negative cognitions interacted with working memory capacity. In each of these models the variables were centered before calculating the interaction term and a setwise hierarchical procedure was used. In Step 1 gender, age, and school attended were entered into the regression equation as background variables. In Step 2 the SOPT total sum score, the relevant (positive arousal, positive sedation or negative) IAT D-measure and the relevant (positive arousal, positive sedation or negative) expectancy sum score were added to the regression equation. In Step 3 the interaction between the relevant (positive-arousal, positive sedation or negative) expectancy sum score and the SOPT total sum score was added to the model. In Step 4 the interaction between the relevant (positive arousal, positive sedation or negative) IAT D-measure and the SOPT total sum score was added to the model.

Table 3

Summary of Simultaneous Regression Analysis for Variables Predicting Alcohol Use After One Month $(\mathrm{N}=81)$

\begin{tabular}{l|l|l|l|l}
\hline Variable & $B$ & SE B & \multicolumn{1}{l}{$\beta$} \\
\hline Gender & .37 & .16 & $.24^{*}$ \\
\hline Expl. Active & .11 & .09 & .14 \\
\hline IAT Active & .02 & .11 & .02 \\
\hline WMC & .02 & .03 & .09 \\
\hline WMC X Expl. Active & .10 & .04 & $.28^{* *}$ \\
\hline WMC X IAT Active & -.10 & .04 & $-.29^{* *}$ \\
\hline
\end{tabular}

Note. Expl. Active $=$ explicit positive-arousal alcohol-related cognitions; IAT Active = Implicit Association Test d2SDscore expressing the strength of the positive arousal association with alcohol; $W M C=$ working memory capacity expressed in total number of correct responses on three consecutive Self Order Pointing Tasks. ${ }^{*} p<.05 * * p<.01$ 


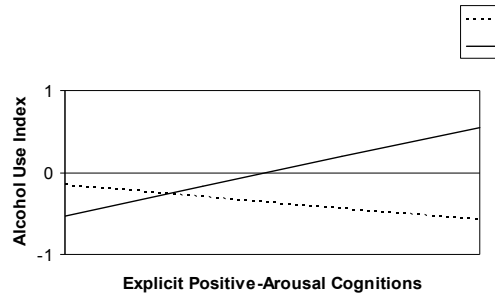

Figure 3. Interaction between Working Memory Capacity and Explicit Positive-Arousal Cognitions in the Prediction of Alcohol Use $(B=.28, p<.01)$. Alcohol Use Index = log transformed standardized sum score of eight outcome variables, Explicit Positive-Arousal Cognitions $=$ explicit positive-arousal alcohol expectancies score, $\mathrm{WMC}=$ working memory capacity score expressed in total number of correct responses on three consecutive Self Order Pointing Tasks.

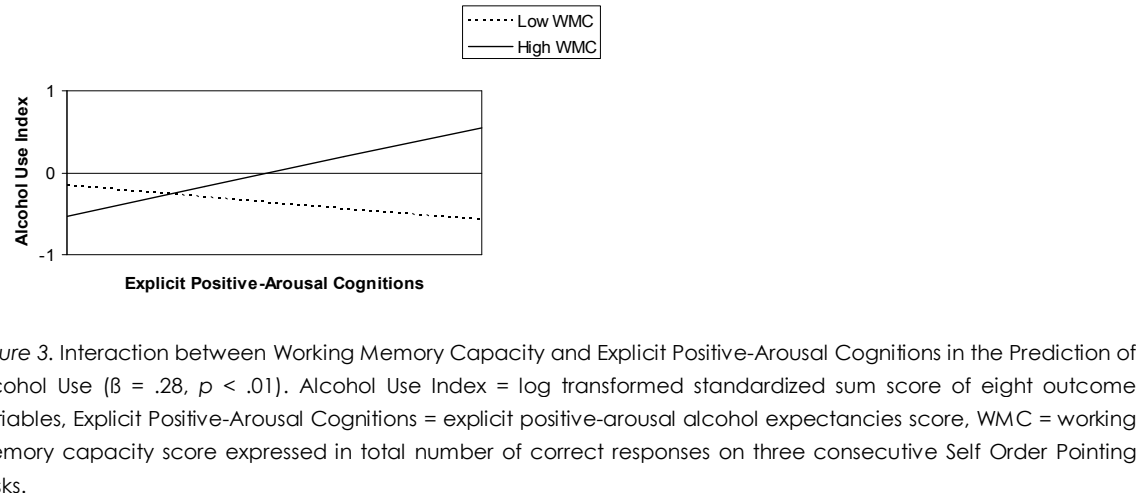

The alpha level was set at .05 for all analyses to ensure an optimal trade-off between completeness (not leaving out possibly interesting effects) and the correctness (restricting Type-Il error) given the exploratory nature of the data. The term borderline significant was used when the $p$-value exceeded .05 but was less than .10 .

Only the model, in which the interactions between explicit and implicit alcoholrelated cognitions and working memory capacity reached borderline significance in the prediction of alcohol use, was subjected to further analysis. Both interactions added significantly to the prediction of one-month follow-up alcohol use (Step $3 \Delta R^{2}=.06, p=$ .02 , Step $4 \Delta R^{2}=.05, p=.02$ ) above and beyond the background variables and main effects (see Table 2). Overall, the full model explained $31 \%$ of all variance ( $R^{2}$ adjusted $=.21, F(10,70)=3.08, p<.01)$. A trimmed model was obtained by removing all variables that were not (borderline) significant from the regression equation. The final trimmed model shows that both the interaction between working memory capacity and explicit positive-arousal cognitions and the interaction between working memory capacity and implicit positive-arousal cognitions predicted alcohol use after one month controlling for gender (see Table 3). Figure 2 shows that in participants with low 
working memory capacity, the implicit positive-arousal associations positively predicted one-month follow-up alcohol use. However, in the participants with high working memory capacity the opposite appeared to be true. In contrast Figure 3 shows that in participants with high working memory capacity, stronger explicit positive- arousal cognitions predicted greater alcohol use. In those with low working memory, there was no such relation between explicit positive arousal expectancies and subsequent alcohol use. Overall, the final trimmed model explained $27 \%$ of all variance $\left(R^{2}\right.$ adjusted $=.21, F(6,74)=4.55 p<.001)$.

Additionally, the analysis was repeated controlling for baseline drinking. In this analysis the interaction between implicit positive-arousal cognitions and working memory capacity was no longer significant $\left(\Delta R^{2}=.02, p=.44\right)$. This is not surprising, given the limited power of this analysis. Note that it is a different question whether change in drinking overtime can be predicted by an interaction between working memory capacity and implicit or explicit alcohol-related cognitions, or whether drinking behavior itself can be predicted by an interaction between working memory capacity and implicit or explicit alcohol-related cognitions. This study primarily evaluated implicit and explicit cognitive processes and interactions between specific cognitive processes and working memory in the prediction of one-month follow-up drinking.

Table 4

Means and Standard Deviations of Explicit Cognition, Implicit Cognition and Working Memory Capacity

\begin{tabular}{l|ll}
\hline & $\begin{array}{l}\text { Low WMC } \\
(\mathrm{N}=48)\end{array}$ & $\begin{array}{l}\text { High WMC } \\
(\mathrm{N}=33)\end{array}$ \\
\hline Expl Active & $\begin{array}{l}4.18 \\
(0.88)\end{array}$ & $\begin{array}{l}4.43 \\
(1.07)\end{array}$ \\
\hline Expl Relaxed & 4.11 & 3.94 \\
& $(1.01)$ & $(0.76)$ \\
\hline Expl Miserable & 2.19 & 2.29 \\
\hline IAT Active & $(0.96)$ & $(0.93)$ \\
\hline IAT Relaxed & 346.48 & 387.13 \\
& $(229.57)$ & $(244.97)$ \\
\hline IAT Miserable & 303.12 & 351.04 \\
& $(229.37)$ & $(232.50)$ \\
\hline WMC & 397.68 & 466.55 \\
\hline
\end{tabular}

Note. Expl Active = explicit positive-arousal alcohol cognitions on a 6-point Liker scale; Expl Relaxed = explicit positivesedation alcohol cognitions on a 6-point Liker scale; Expl Miserable = explicit negative alcohol cognitions on a 6point Liker scale; IAT Active = positive-arousal IAT score in ms; IAT Relaxed = positive-sedation IAT score in ms; IAT Miserable = negative IAT score in ms; WMC = working memory capacity expressed in total number of correct responses on three consecutive Self Order Pointing Tasks; Low WMC = participants who scored low on working memory capacity based on a median split; High WMC = participants who scored low on working memory capacity based on a median split. 


\section{DISCUSSION}

This study showed that the interaction between implicit and explicit positive-arousal cognitions and working memory capacity predicted one-month follow-up alcohol use in our sample of at-risk adolescents. Results from a hierarchical regression analysis showed that both interactions predicted unique variance in one-month follow-up alcohol use after controlling for the main effects and background variables. Interestingly, the signs of these interactions were in the opposite direction. That is, implicit positive-arousal cognitions predicted one-month follow-up alcohol use more strongly in students with lower levels of working memory capacity, whereas explicit positive-arousal cognitions predicted one-month follow-up alcohol use more strongly in students with higher levels of working memory capacity.

These results are in line with prior research on moderating effects of executive functions on implicit cognitive processes. Implicit cognition has been shown to be predictive of behavior in individuals with relatively low working memory capacity or attentional control (Grenard et al, in press; Payne, 2005). In addition, our findings are consistent with the findings of Tapert et al (2003) that explicit positive alcohol expectancies predicted alcohol use in substance use disorder adolescents with good verbal skills, whereas they did not in substance use disorder adolescents with poor verbal skills. Both findings indicate that alcohol expectancies may require deep processing in order for them to affect decision making and thus drinking. Finally, our results are in agreement with research that found dissociations between implicit and explicit cognitions. For instance, prior studies have shown that explicit cognitions predicted more deliberative behavior whereas implicit cognition predicted more spontaneous behavior (Asendorpf, Banse \& Mücke, 2002; Perugini, 2005). Our results are also congruent with the idea that implicit and explicit cognitions influence behavior through different pathways (e.g. Fazio \& Towles-Schwen, 1999; Kahneman, 2003; Strack \& Deutsch, 2004), which clearly has relevance for addictive behaviors (see Wiers \& Stacy, 2006).

Given some limitations, these results should be interpreted with some caution. First, although we screened for schools with a high proportion of at-risk adolescents, we did not use a probability sampling strategy at an individual level and therefore we might not be able to generalize these results to other at-risk adolescent populations. In addition, we did not have the statistical power to adequately look at possible interactions with other background variables such as gender. It could be that for specific subgroups of at-risk adolescents the results would have been different.

Second, some authors question the validity of the IAT (e.g. Rothermund \& Wentura, 2004; De Houwer, 2001). It has been proposed that participants performing the IAT may recode the stimuli based on other features than the associations the IAT intends to measure. Participants may sometimes select one 'yes'- or 'figure'-category and one 'no'- or 'ground'-category based on salience. This is referred to as the so-called figureground asymmetry (Rothermund \& Wentura, 2004). This figure-ground asymmetry has 
been shown to sometimes influence IAT effects. However, in alcohol-IATs similar to the ones used here, this figure-ground asymmetry could only partly explain the results found for the negative associations and not at all for the positive and arousal associations (Houben \& Wiers, 2006). Moreover, since we mixed conceptual and perceptual task characteristics within one test, we may have been able to provide some defense against possible recoding given the fact that not just one simple recoding strategy can be used. Since participants do not react just to one dimension of interest, the task may be less prone to response strategies the participants might want to employ (cf. De Houwer, 2003; Rinck \& Becker, 2007). One could further argue that executive functions directly influence the estimates of associations provided by the IAT, for example through switch costs (Mierke \& Klaver, 2003). However, we used the new scoring algorithm here (Greenwald et al., 2003), which provides an estimate of associations, while accounting for individual differences in switching capabilities (Greenwald et al., 2003; Mierke \& Klaver, 2003). The hypothesis that the IAT results here are strongly influenced by the level of executive functions in this study is further unlikely given the lack of correlation between the IAT estimates of positive-arousal associations and working memory capacity. In addition, a similar pattern of results was found by Grenard et al. (in press) when using a different (not a reaction time task) and uncorrelated indirect measure of implicit alcohol-related cognitions (see Ames et al., 2007 for a direct comparison). Additionally, there is converging evidence from other research domains: Payne (2005) found a similar interaction between the IAT and executive control predicting the stereotyping behavior, and Hofmann, Rauch and Gawronski (2007) found a similar interaction between appetitive associations assessed with a different variety of the IAT and experimentally manipulated levels of self regulatory control predicting eating behavior.

Third, since the IAT, SOPT and alcohol expectancy questionnaire were performed in a fixed sequence, it is possible that order effects may have played a role in the current results. However, this fixed sequence was chosen as the most optimal procedure to minimize method-related variance in a study focusing on individual differences (cf. Asendorpf et al., 2002).

Fourth, a possible limitation is that the follow-up period was relatively short. This places some restrictions on the prospective nature of this data. Further research is necessary to determine whether these interactions between alcohol-related cognition and working memory capacity are capable of predicting alcohol use in at-risk adolescents over longer time periods.

Finally, it must be noted that since the relationship between executive functions and alcohol use has been shown to be bidirectional it may be hard to disentangle the precise causal influences in the current pattern of results. For instance, it could well be that, due to prior drinking, working memory capacity is lowered and causes positivearousal associations with alcohol to influence drinking behavior to a greater extent. This, in turn, could lead to heavier drinking and more alcohol-related problems. Yet, al- 
though prior research has shown that that poor executive functioning has been associated with more alcohol-related problems (e.g. Finn \& Hall, 2004; Peterson et al., 1992; Tapert et al., 2002), in our sample the SOPT did not correlate significantly with the RAPI. This could be due to the fact that the average item score on the RAPI of .30 was not that high compared to a clinical sample (White \& Labouvie, 1989). This is in line with prior studies that have shown that adolescents may experience the negative sedative effects of alcohol, which may serve to limit intake, less. Nevertheless these adolescents seem to be more sensitive than adults to alcohol-induced damage in structures that should restrict e.g. binge drinking (Spear, 2004). Additional research with longitudinal designs or more experimental designs are necessary to separate the bidirectional effects between executive functioning and alcohol use.

In sum, although these limitations should be taken into consideration when interpreting these results, this study does provide additional information for the role of alcohol-related cognitions in the development of (hazardous) drinking behavior. These results suggest that implicit and explicit alcohol-related cognitions influence drinking behavior through different pathways: adolescents with relatively good working memory capacity appear to make more "reasoned" drinking decisions, while adolescents with relatively poor working memory capacity appear to make more impulsive drinking decisions (cf. Strack \& Deutsch, 2004). The obtained interaction effects, and especially the obtained dissociation in these effects, should be considered important given that interaction effects of continuous variables in field studies are often difficult to detect (McClelland \& Judd, 1993). Because effect sizes are often attenuated in such analyses and thus notoriously difficult to uncover, McClelland and Judd (1993) concluded that it is more important to determine if an interaction effect exists than to focus on its effect size.

Thus, both the interaction between working memory capacity and implicit positivearousal cognitions with alcohol and the interaction between working memory capacity and explicit positive-arousal cognitions could be taken into account when prevention and intervention methods are being developed. Different intervention methods might be effective for different subgroups of at-risk adolescents. On the one hand, at-risk adolescents with good working memory capacity might benefit from interventions that try to strengthen protective negative alcohol cognitions, such as a motivational interview, which appears feasible in at-risk adolescents (Grenard et al., 2007). On the other hand, at-risk adolescents with poor working memory capacity might benefit from interventions that attempt to directly interfere with their automatic reactions, such as an attentional retraining (e.g. Wiers, Cox et al., 2006) or with interventions that aim at increasing their capacity to regulate impulses, such as a working memory training, as has been successfully used in children with ADHD (Klingberg et al., 2005). However, it should be noted that even if an increase in working memory capacity could also be achieved in high-risk adolescents, this does not replace the importance of motivation and goals: the adolescent still needs to be motivated or have a goal in mind to apply 
this executive control (Feldman-Barrett et al., 2004; Wiers et al, 2007). Future research is needed to indicate if and which methods can be included as proven effective components in intervention programs. 
APPENDIX A

\section{IAT picture stimuli}

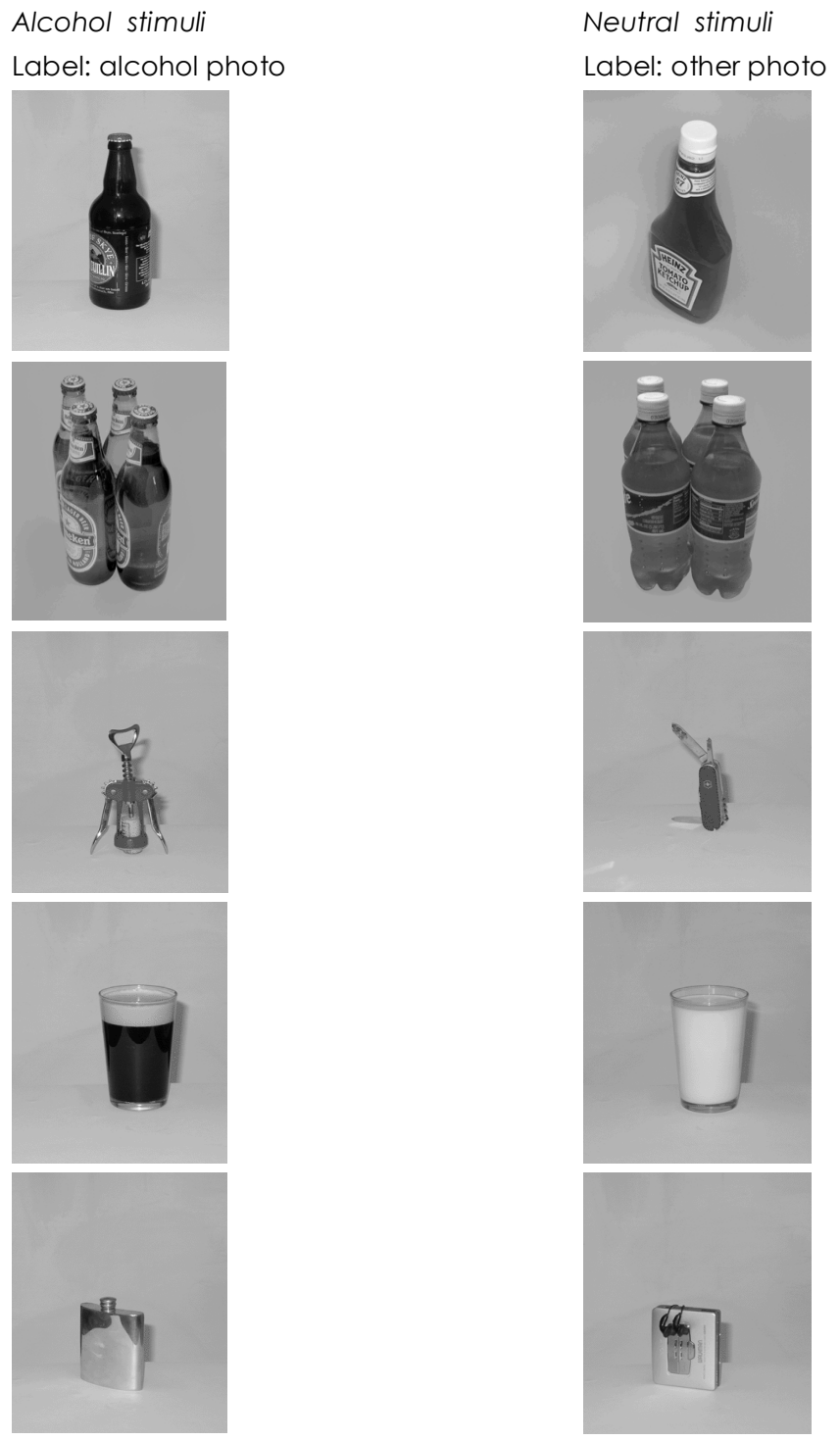




\section{APPENDIX B}

\section{IAT word stimuli}

(Translated from Dutch)

Positive Arousal stimuli
(active word)
excited
energetic
busy
lively
wild

Neutral stimuli

(neutral word)

historical

apart

steep

wide

compact

Positive Sedation stimuli

Neutral stimuli(

relaxed word)

(neutral word)

relaxed

normal

calm

blue

chill

flat

tranquil

central

comfortable

digital

Negative stimuli

Neutral stimuli

(miserable word)

(neutral word)

sad

daily

pain

square

sick

nauseous

narrow

common

miserable

totally 



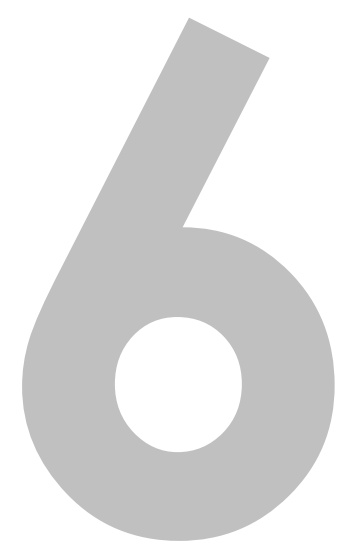

The influence of Motivational Interviewing on explicit and implicit alcohol-related cognition and alcohol use in at-risk adolescents

Brief report published as: Thush, C. , Wiers, R.W., Moerbeek M., Ames, S. L. , Grenard, J. L. , Sussman, S. , \& Stacy, A.W. (in press). The influence of Motivational Interviewing on explicit and implicit alcohol-related cognition and alcohol use in at-risk adolescents. Psychology of Addictive Behaviors 


\section{ABSTRACT}

Both explicit and implicit cognitions play an important role in the development of addictive behavior. This study investigated the influence of a single session Motivational Interview on explicit and implicit alcohol-related cognition and whether this intervention was successful in consequently decreasing alcohol use and alcohol-related problems in at-risk adolescents. Explicit and implicit alcohol-related cognitions were assessed at pretest and one month post-test in 125 Dutch at-risk adolescents ranging in age from 14 to 23 (51 males) with an expectancy questionnaire, an adapted version of the Implicit Association Test (IAT) and a dual change Flicker Task. Motivation to change, alcohol use and alcohol-related problems were measured with self-report questionnaires, at pre-test, at post-test after one month and at the six-month follow-up. The results did not yield support for any differential effects of the intervention on drinking behavior or readiness to change at post-test and six-month follow-up. There were however changes in alcohol-related cognitions between pre- and post-test. Overall, the quality of the brief intervention was rated positively and these results warrant further investigation. 
Dual process models predict that both explicit reflective and implicit cognitive processes together influence behavior (e.g. Fazio \& Towles-Schwen, 1999; Kahneman, 2003; Strack \& Deutsch, 2004). Implicit cognitions represent more automatic underlying motivational processes, whereas explicit cognitions are related to slower deliberate thought processes (Greenwald \& Banaji, 1995; Kahneman, 2003). In addiction research, explicit alcohol expectancies have been shown to be predictive of concurrent and, to a lesser extent, prospective alcohol use (e.g., Goldman \& Darkes, 2004; Jones, Corbin \& Fromme, 2001; Sher, Wood, Wood, \& Raskin, 1996; Stacy, Newcomb, \& Bentler, 1991). Implicit cognitions have been shown to predict unique variance in current and prospective alcohol use after controlling for explicit cognitions (e.g. Ames et al., 2007; Jajodia \& Earleywine, 2003; Stacy, 1997; Thush \& Wiers, 2007; Wiers, van Woerden, Smulders, \& De Jong, 2002).

An assumption of dual process models is that the influence of implicit processes on subsequent behavior can be moderated by explicit processes if motivation and the opportunity to do so are high (e.g. Fazio \& Towles-Schwen, 1999; Kahneman, 2003; Strack \& Deutsch, 2004). Indeed, neurobiological addiction research has shown the prefrontal cortex and associated areas involved in more reflective decision making and in the moderation of impulses (e.g. Bechara, 2005; Kalivas \& Volkow, 2005). Dual process models of addiction predict that the influence of implicit appetitive cognitions on subsequent addictive behavior are dependent on moderators such as motivation and executive functioning (e.g. Stacy, Ames, \& Knowlton, 2004; Wiers et al, 2007). In line with this notion, recent studies indicated that the impact of implicit alcohol cognitions on alcohol use in at-risk adolescents was moderated by working memory capacity: implicit alcohol associations were found to be better predictors of alcohol use in adolescents with relatively poor working memory than in adolescents with relatively good working memory (Grenard et al., 2007; Thush et al., 2008). In addition to the ability to refrain from more impulsive use of alcohol, it is also important that adolescents are motivated to do so. Indeed, prior research has shown that the influence of implicit processes on behavior increases when motivation not to act on these automatic associations is low (Olson \& Fazio, 2004). The present study sought to increase control over more automatic influences on alcohol use in at-risk adolescents by increasing motivation in order to moderate use of alcohol.

The importance of having adaptive motivational structures in the context of changing one's tendencies toward more addictive behaviors has been demonstrated by a large body of research (Miller \& Rollnick, 2002). Having more adaptive motivational structures can be defined, for example, as having positive goals in life that an individual wants to achieve, feeling optimistic about achieving these goals, and the satisfaction that will ensue. Research showed that individuals with more adaptive motivational structures demonstrated that they are better able to control their drinking than those with maladaptive motivational structures. Additionally, research showed that in treatment alcohol abusers with adaptive motivational structures show a more 
immediate response to treatment, less problem denial and more motivation for change than those with maladaptive motivational structures (Cox \& Klinger, 2002).

Indeed, interventions that consist of brief Motivational Interviewing (Ml; Miller \& Rollnick, 2002) have consistently yielded greater support for their efficacy to reduce hazardous drinking in adults compared with educational or information-only interventions (e.g. Marlatt et al., 1998, see for review, Larimer \& Cronce, 2002; 2007). However, although brief $\mathrm{MI}$ has been proven to be effective in reducing hazardous drinking in (young) adult populations, there is as yet little evidence for its' effectiveness in (at-risk) adolescents, although it should be noted there is little research yet (for review see Grenard, Ames, Pentz \& Sussman, 2006). Motivational interventions might still be one of the most promising approaches especially in difficult at-risk non-treatment seeking populations (Grenard et al., 2007; Moyer, Finney, Swearingen, \& Vergun, 2002).

Besides the limited data regarding the effectiveness of $\mathrm{Ml}$ in at-risk adolescents, a second reason to perform the current study is that there is still much unknown about the cognitive mechanisms underlying $\mathrm{Ml}$ effects. Modifying readiness to change is seen as an important precursor to behavior change (Miller \& Rollnick, 2002). Therefore, increasing readiness to change might be an important underlying mechanism through which MI works. However, only a few studies have tried to identify the cognitive mechanisms through which MI might work (e.g. Bosari \& Carey, 2000; Wood, Capone, Laforge, Erickson \& Brand, 2007). To our knowledge, the influence of MI on explicit and implicit alcohol-related cognitions in combination with motivation to change and subsequent drinking behavior in at-risk adolescents has not been studied. Therefore, this study could increase our understanding of the underlying cognitive mechanisms influenced by MI.

This study investigated the influence of $\mathrm{MI}$ on explicit and implicit alcohol-related cognitions (i.e., implicit associations and attentional bias) and alcohol use in at-risk adolescents in a randomized field study. It was hypothesized that in the experimental group motivation to change and negative expectancies (two related concepts, Jones \& McMahon, 1998) would increase compared to the information-only control group. Second, it was hypothesized that the experimental group would decrease their drinking compared with the control group. Third, the intervention was not expected to directly affect implicit alcohol-related cognitions, but rather that a reduction in drinking would be achieved through a moderating effect of an increased motivation to change (Stacy et al., 2004; Wiers et al., 2007).

\section{METHOD}

\section{Participants}

A total of 125 Dutch adolescents (51 male and 74 female) were included at pre-test and randomly assigned to the experimental intervention group $(n=61)$ and informa- 
tion-only control group ( $n=64$ ). Participants were in the age range of 15 to 23 (mean age $=17.07, S D=1.64$ ) and were recruited from five low-level vocational schools. Based on a Dutch national health survey among high school adolescents, low-level vocational schools showed the highest prevalence of alcohol and drug use as well as behavioral problems. Hence at the group level, these students are at risk for developing substance-related problems. Participants drank an average of 2.57 Dutch standard alcoholic drinks on a weekend day $(S D=3.66)$ and an average of 0.30 Dutch standard alcoholic drinks on a week day $(S D=1.28$ ) (a standard alcohol serving in the Netherlands contains 10 grams of alcohol). Out of the total sample 80 participants (64\%) indicated having one or more binge drinking episodes (five or more Dutch standard alcoholic drinks on one occasion) in the last month.

\section{Material}

\section{Alcohol Use}

Alcohol use was assessed with a Dutch version of the alcohol use questionnaires as described in Ames et al. (2007). Self-report questionnaires have been proven to be reliable and valid if participant sobriety and confidentiality of data are assured (Sobell \& Sobell, 1990). Both requirements were fulfilled in this study. Participants were asked how many times they used various drugs in the last month on an 11-item rating scale (ranging from 'never used' to '91-100+ times'). Participants respond to a list of specific drugs (i.e. alcohol, marijuana, ecstasy, etc.) or more general drug categories (i.e. hallucinogens, stimulants, other club drugs, etc.). Additionally, participants indicated how many Dutch standard glasses of alcohol they consumed on each day of the past week. Additionally, they had to indicate on a 6-point Likert scale on how many occasions they drank five Dutch standard glasses of alcohol or more in the past month (ranging from 'I do not drink' to 'seven times or more').

\section{Alcohol-Related Problems}

An index of alcohol-related problems was assessed using an adapted version of the Rutgers Alcohol Problems Index (RAPI; White \& Labouvie, 1989). We used the 18-item version of the RAPI which correlated .99 with the original 23-items version (White \& Labouvie, 2000). Participants were asked to indicate on a 5-point Likert scale (ranging from 'never' to 'daily') how many times they experienced certain problems within the last months because of their alcohol use. An example of an item is: "Not able to do your homework or study for a test". The items were summed to create an index of alcohol-related problems (Cronbach's alpha =.79).

\section{Implicit Association Test}

In the IAT, participants categorize four categories of stimuli as quickly as possible while only using a left or right response key (see Greenwald, McGhee, \& Schwartz, 1998). In 
this study, three adapted versions of the original IAT were used in which both stimulus pictures and words were used (see Figure 1). One IAT assessed the association between 'active' positive arousal words versus 'neutral' words with photos of objects related to alcohol or objects related to soft drinks. Another IAT assessed the association between 'relaxed' positive sedation words versus neutral words with alcohol related objects or soft-drink related objects. Lastly, one IAT assessed the association between 'miserable' negative words versus neutral words with words with alcohol related objects or soft-drink related objects. These attribute categories were chosen because they represent the three main categories of alcohol expectancies (Goldman \& Darkes, 2004). The stimulus pictures used were matched on size, background, shape and color of the object (see Appendix A). The words used (see Appendix B) were matched on number of letters, syllables, familiarity, and on valence and arousal values. In this study the three IATs were programmed in ERTS 3.18 (Beringer, 1996). Each attribute discrimination block consisted of 20 trials and each combination block consisted of 40 trials. The D-2SD penalty score for practice and test was chosen as the main reaction time measure (see Greenwald, Nosek, \& Banaji, 2003). The internal consistency between practice and test items was acceptable (Cronbach's alpha .57 to .64).

\section{Dual Change Flicker Task}

In the original flicker paradigm, two identical stimulus arrays are presented in rapid, continuous succession while separated by a mask (Rensink, 2002). A permanent change is made to one of the stimulus arrays and while the two stimulus arrays are flicked back and forth, it is the participants' task is to detect the change as quickly as possible. If the change is made to an alcohol object in a scene, the time needed to detect the change is assumed to measure an attentional bias for alcohol (Jones, Jones, Blundell, \& Bruce, 2002; Jones, Jones, Smith, \& Coply, 2003). In the current task, the two stimulus arrays consisted of pictures of alcoholic drinks and soft drinks in a $3 \times 6$ rectilinear matrix. On one side $3 \times 3$ alcohol pictures are presented whereas on the other side 3 $x 3$ soft drinks pictures are presented. In this version of the flicker paradigm, two changes were made to the pictures in the center of the $3 \times 3$ matrixes (see Appendix C). However, in the instructions, participants were told that a change would be made and it was their task to detect this one change by pressing the right or left key depending on which side they detected the change. This procedure might be a more sensitive way of detecting attentional biases than using a single change, since simultaneously presented changes will compete for the participants' attention (Jones et al., 2002). The original stimulus was presented for $250 \mathrm{~ms}$., subsequently a mask appears for $80 \mathrm{~ms}$., followed by the changed stimulus (an alcohol and soft drink change) for $250 \mathrm{~ms}$. and the mask for $80 \mathrm{~ms}$. . The stimulus pictures used were matched on size, background, shape and color of the object. The four versions of the task were programmed in ERTS 3.18 (Beringer, 1996). 


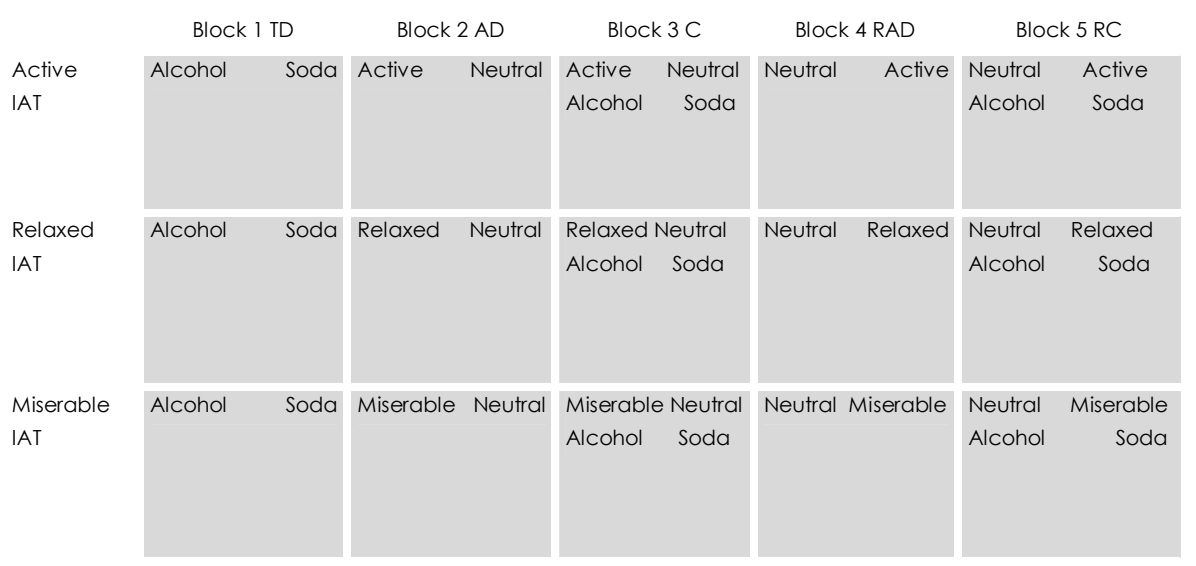

Figure 1. Schematic overview of the block sequence in the three Implicit Association Tests. AD = Attribute Discrimination, $C=$ Combination, $R A D=$ Reversed Attribute Discrimination, $R C=$ Reversed Combination.

\section{Expectancy Questionnaire}

The direct measure of alcohol-related cognitions was 18-item expectancy questionnaire representing an explicit version of the implicit test words (see Appendix B; as in Wiers et al. 2002). Each item consisted of a statement on drinking alcohol (for example: 'drinking alcohol makes me feel energetic'). Participants indicated the extent to which they (dis)agreed with each item on a 6-point Likert scale. The questionnaire consisted of three scales: a positive-arousal, a positive-sedation and a negative outcome scale. The internal consistency of all three scales was good (Cronbach's alphas ranging from .70 to .87$)$

\section{Readiness to Change}

Readiness to change was measured with ruler specifically designed for this purpose (LaBrie, Quinlan, Schiffman, \& Earleywine, 2005). The anchors for the 10-point readinessto-change ruler were "I never think about my alcohol/drug use" and "My alcohol /drug use has changed. I now drink/use drugs less."

\section{Procedure}

\section{Recruitment}

Participants were recruited through five minute classroom enrollment talks, which explained that students could participate in a study about what young people do in there spare time. It was not mentioned that this study was related to the topic of alcohol use or changing behavior in order to prevent priming the participants towards alcohol-related responses on the IAT and Flicker Task. 
Pre-test

After obtaining active consent from the participants (and if the participant was a minor also the parents), participants were tested in groups of four at school in a separate test room during school time. The Flicker Task was administered first on a laptop with a separate response device. Four versions of the dual change Flicker Tasks were used counterbalancing the alcohol picture array on the left or the right side and two different sets of changes. Subsequently, the three IATs were partially counterbalanced administered on the same laptop with the separate response device. Throughout the trials of the IATs, participants received feedback in red letters on their screen (incorrect response= 'ERROR', > 3000 ms. = 'TOO SLOW', < 150 ms. = 'TOO FAST'). The indirect measures were administered before the direct measure, because of possible carry-over effects, which are stronger when the direct test is administered first (Bosson, Swann \& Pennebaker, 2000). Subsequently, the participants filled out the expectancy questionnaire. The alcohol use and alcohol-related problems questionnaires and readiness-tochange ruler were administered last, in order to avoid any interference between having to report ones alcohol use, alcohol-related problems or motivation to change and the indirect and direct measures of alcohol-related cognitions. The total assessment took approximately 45 minutes for each participant. Directly after, participants were randomly assigned to the intervention or control group.

\section{Intervention}

The intervention consisted of a brief $\mathrm{Ml}$ in which normative feedback on adolescent substance use was provided. During a Ml session various counseling skills are used (such as reflective listening, affirmations, open ended questions and summaries) in order to elicit 'change talk' (Miller \& Rollnick, 2002). This creates an opportunity for the participants to think and talk about their own behavior (also in comparison to others), high-risk situations, the advantages and negative consequences of their behavior. The main objective is to stimulate new thinking on their personal substance use, which might result in behavioral change. The interviews were conducted by psychology graduate students who were trained in Ml, utilizing a written protocol during the session. The intervention consisted of several key elements. First, after the student was greeted and confidentiality was explained, a couple of minutes were spent on establishing rapport. Second, the interviewer and student agreed upon a behavior to discuss (options included use of several specific substances or other issues related to the students' spare time or night out). Third, age and gender specific normative feedback was provided when appropriate. Fourth, the advantages and disadvantages of the current drugs used were discussed by using a decisional balance sheet and/or by using importance and confidence scales. Fifth, if appropriate the student's capacity and strategies to change were discussed and affirmed. The session was then summarized by the interviewer and the student received a one dollar banknote. It was explained that this study was conducted in cooperation with researchers in the US and that the one dollar note 
was a token of our appreciation for the student's assistance in this international project and secondly that it might help them to remember the interview and the issues that were discussed. The interview was conducted in approximately 30 minutes. After each interview, an evaluation questionnaire was administered separately to the student and interviewer. The student had the possibility on the last page of this evaluation form to state a pledge for him or herself. Lastly, the student in the experimental group received five information flyers about the biological and cognitive effects of alcohol, tobacco, marijuana, ecstasy and other stimulant drugs.

\section{Information-Only Control Group}

After filling out the pre-test, participants in the control group received five information flyers about the biological and cognitive effects of alcohol, tobacco, marijuana, ecstasy, and other stimulant drugs. On the backside of the flyers, the address of the appropriate community health center and several drug information websites and telephone lines were listed.

\section{Post-test}

One month later, the participants followed the same assessment procedure as was followed at pre-test. Additionally, participants were asked to provide their home address if they agreed to being contacted again. Once more the confidentiality of the data was guaranteed. Out of the 125 participants 113 participants $(90.40 \%)$ were present at post-test.

\section{Follow-up}

Approximately six months after the intervention, participants who agreed to being contacted again, were contacted by mail. In an accompanying letter the confidential and anonymous nature of the data was emphasized and the participants were asked to fill out the enclosed in questionnaires (alcohol use and alcohol-related problems questionnaires and readiness-to-change ruler) and to return them in a prepaid envelope. Out of the 113 participants at post-test, 103 agreed to be contacted again. Out of that sample, 74 participants $(71.8 \%)$ returned the filled out questionnaire.

\section{Data reduction}

In order to obtain a normally distributed dependent variable and to reduce the chances of a Type I error by multiple testing, we computed a log transformed standardized alcohol use index score in three consecutive steps. First, $z$ - scores were calculated for six different correlated outcome measures, namely number of times alcohol used in the past month, the mean number of standard drinks on a weekend day, the mean number of standard drinks on a week day, the number of binges in the last week, frequency of binges per month and the total sum score on the RAPI. Subsequently, the 
alcohol use index score was computed by calculating the mean of these six z-scores. Finally, the alcohol use index was log transformed to obtain a normally distributed dependent variable (Cronbach's alpha .85).

All short-term effects of the intervention on implicit and explicit alcohol-related cognition, readiness to change and the alcohol use index were analyzed from pre-test to one month post-test. The long-term effects of the intervention on the alcohol use index were analyzed from pre-test to one-month post-test and six-month follow-up.

\section{RESULTS}

\section{Drop Out and Outliers}

Out of the 61 participants included in the experimental group, only 6 participants did not discuss alcohol use to a great extent2. A total of 113 out of 125 participants (90\%) stayed in the program and completed the post-test assessments. Out of the 12 participants who dropped out at post-test, 5 participants belonged to the control group and 7 participants belonged to the experimental group $\left(\chi^{2}(1)=0.48, p=.49\right)$. This indicates that there was no evidence for selective drop out. Moreover, the participants and drop outs did not differ significantly in their alcohol use. After six months, according to the intention to treat principle, 74 of the 125 participants (59\%) returned the filled out followup questionnaires. Using a more lenient and realistic criterion, out of 103 participants who agreed at post-test to be contacted again, 74 participants $(72 \%)$ returned the filled out questionnaire. Participants and drop outs differed significantly in their baseline alcohol use; the drop outs had a lower alcohol use than the participants.

Analyses included only those participants reporting that they had consumed alcohol in their lifetime: 15 participants reported that they had never consumed alcohol and were therefore eliminated from all further analyses. Additionally, the scores on the IAT of 7 participants who exceeded the mean error scores in the specific reaction time task by more than 3 standard deviations were excluded from further analyses that concerned that specific IAT (see Greenwald et al., 2003). Furthermore, the scores on the Flicker Task of 9 participants who reported having seen two changes during the Flicker task were excluded from further analyses (see Jones et al., 2002). Thus, the final analytical sample at pre-test consisted of 110 participants (experimental group $=55$; control group $=55$ ), at post-test of 100 participant (experimental group $=50$, control group $=50$ ) and at follow-up of 70 participants (experimental group $=36$, control group $=34)$.

2 Given that the results did not differ for the analytical sample with or without these participants, we decided to include these participants to have more statistical power to conduct the long term analyses. 
Table 1

Pearson Correlation for Implicit and Explicit Cognition and Alcohol Use at Pre-test

\begin{tabular}{|c|c|c|c|c|c|c|c|c|c|}
\hline & 1 & 2 & 3 & 4 & 5 & 6 & 7 & 8 & 9 \\
\hline 1. Expl Active & - & & & & & & & & \\
\hline 2. Expl Relaxed & $.26^{* *}$ & - & & & & & & & \\
\hline 3. Expl Miserable & -.09 & -.11 & - & & & & & & \\
\hline 4. IAT Active & -.01 & .05 & .01 & - & & & & & \\
\hline 5. IAT Relaxed & .02 & -.03 & .01 & .18 & - & & & & \\
\hline 6. IAT Miserable & -.08 & .04 & .08 & .06 & .15 & - & & & \\
\hline 7. Flicker Task & -.03 & .07 & .12 & -.03 & -.11 & -.15 & - & & \\
\hline 8. Readiness To Change & -.12 & $.20^{*}$ & -.04 & -.07 & .02 & -.13 & -.17 & - & \\
\hline 9. Drink Index & $.22^{*}$ & $.24^{*}$ & $-.31^{* *}$ & $.26^{* *}$ & .19 & .05 & -.18 & $.20^{*}$ & - \\
\hline
\end{tabular}

Note. Expl Active = explicit positive-arousal alcohol cognitions; Expl Relaxed = explicit positive-sedation alcohol cognitions; Expl Miserable = explicit negative alcohol cognitions; IAT Active = D-2SD score for the positive-arousal IAT; IAT Relaxed = D-2SD score for the positive-sedation IAT; IAT Miserable = D-2SD score for the negative IAT; Flicker Task = the attentional bias score reflecting if an alcohol change was detected or not; Readiness To Change = score on the readiness-to-change ruler; Drink Index = log-transformed standardized index score of number of times alcohol was consumed in the last month, the mean number of standard drinks on a week day and on a weekend day, the number of binges per week, number of binges in the past month and the sum score of alcohol-related problems. $P$ $<.05$, two-tailed. ** $p<.01$, two-tailed.

\section{Bivariate Analyses}

The alcohol use index was significantly positively correlated with positive-arousal expectancies, positive-sedation expectancies, the positive-arousal IAT score and readiness to change score. Moreover, the alcohol use index was significantly negatively correlated with negative expectancies (see Table 1). The IAT measures, the attentional bias measure and the alcohol expectancy measures were not correlated with each other (all $p>$.15).

\section{Quality of the Intervention}

In order to assess the quality of the conducted interviews, two questionnaires were used in which both the interviewer and the participant rated the quality of the interview. In addition, a small sample of taped interviews $(N=11)$, reflecting about $10 \%$ of the total sample, were rated using the Motivational Interview Treatment Integrity Code (MIT); Moyers, Martin, Manuel, Hendrickson \& Miller, 2005) and rated to establish whether the interviewer followed protocol (e.g. that the Ml contained the element of providing normative feedback). In general, there were no signs that the intervention was not performed according to Ml-standards or protocol; on average the quality of the interviews was good (for an example see Table 2). Detailed data on the quality of the Mls are available from the first author upon request. 
Table 2

Means and Standard Deviations on the Motivational Interview Treatment Integrity Code $(\mathrm{N}=11)$

\begin{tabular}{|c|c|c|}
\hline & Mean & Standard Deviation \\
\hline Empathy & 5.55 & 0.69 \\
\hline Spirit & 5.00 & 0.63 \\
\hline Information & 2.33 & 1.41 \\
\hline MI True & 3.00 & 1.55 \\
\hline MI Untrue & 0.30 & 0.48 \\
\hline Closed Questions & 24.09 & 5.43 \\
\hline Open Questions & 12.85 & 5.06 \\
\hline Simple Reflections & 4.36 & 1.69 \\
\hline Complex Reflections & 1.27 & 1.49 \\
\hline
\end{tabular}

Note. During 20 min of each interview the following variables were rated. Empathy = extent to which the interviewer understood the participants perspective rated on a 7-point Likert scale ranging from low to high; Spirit = extent to which the interviewer showed MI adherence on a 7- point Likert scale ranging from low to high; Information = total number of times information was given; MI True = total number of times interviewer acted according to MI standards ; MI Untrue = total number of times interviewer acted not according to Ml standards; Closed Questions = number of closed ended questions; Open Questions = number of open ended questions; Simple Reflections = number of reflections that expressed understanding or a paraphrase of the participants words; Complex reflections = number of reflections that expressed a deeper value of the participants words.

\section{Short Term Effects of the Intervention}

\section{Alcohol Outcome Expectancies}

A 2 (Time) X 2 (Condition) Mixed ANCOVA controlling for age and gender revealed a borderline significant Time $x$ Condition effect for the explicit positive sedation alcohol expectancies, $F(1,92)=3.78, p=.055$. However, the increase in explicit positive sedation alcohol expectancies between pre- and post-test in the experimental group was not significant, $t(46)=1.02, p=.32$, nor was the decrease in explicit positive sedation alcohol expectancies between pre- and post-test control group $t(48)=-1.56, p=.13$. Contrary to our hypothesis, there was no significant Time $x$ Condition effect for the explicit negative alcohol expectancies, $F(1,92)=1.86, p=.176$.

\section{Implicit Associations}

A 2 (Time) X 2 (Condition) Mixed ANCOVA controlling for age and gender revealed a borderline significant Time $\times$ Condition effect for the implicit positive arousal associations with alcohol, $F(1,89)=3.42, p=.07$. However, both the decrease in implicit positive arousal associations between pre- and post-test in the experimental group and the increase in implicit positive arousal associations with alcohol between pre- and post-test in the control group were not significant (respectively $t(44)=-1.22, p=.23$ and $t(47)=$ $1.47, p=.15)$. 
Attentional Bias

A 2 (Time) X 2 (Condition) Mixed ANCOVA controlling for age and gender revealed a significant Time $\times$ Condition effect for the attentional bias for alcohol, $F(1,85)=12.77, p$ $<.01$. The increase in attentional bias for alcohol between pre- and post-test in the experimental group was (borderline) significant $(t(43)=1.64, p=.10)$. The decrease in attentional bias for alcohol between pre- and post-test in the control group was significant $(t(44)=-3.50, p<.01)$.

\section{Readiness to Change}

A 2 (Time) X 2 (Condition) Mixed ANCOVA controlling for age and gender did not reveal a significant Time $\times$ Condition effect for the readiness to change score, $F(1,85)=$ $0.01, p>.50$.

Alcohol Use

A 2 (Time) X 2 (Condition) Mixed ANCOVA controlling for age and gender did not reveal a significant Time $x$ Condition effect for the alcohol use index, $F(1,93)=0.12, p$ > .50. Given that there was no significant change in the alcohol use index, we did not perform the additional analyses to investigate the possible moderation of motivation on the influence of implicit alcohol association on subsequent drinking behavior.

\section{Long Term Effect of the Intervention}

We analyzed the long term effects of the intervention by constructing a Latent Growth Model (LGM; Meredith \& Tisak, 1990) using AMOS 7.0 (Arbuckle, 2006). LGM methods are a form of Structural Equation Modeling (SEM) and offer several advantages over traditional methods for assessing change over time. First, because LGM methods are able to model both inter- and intra-individual variability in change, they can give a more accurate estimation of the differential effect of condition in change over time. Second, because LGM methods can include several measurement points in outcome assessment, they offer maximized information on the individual change over time compared with simple pre- and post-test designs. An additional advantage is that LGM methods can easily handle missing data, which might be especially useful in the context of analyzing data from the current study (see Hess, 2000).

Although participants were recruited at five different schools, we did not perform a multiple group analysis since some schools had very small sample sizes. We could therefore not estimate and test between-school differences with respect to change in alcohol use. Given that we only had a small sample of five schools, the following results should thus be interpreted with some caution with regard to the generalization of these results to other at-risk adolescent populations. 
Table 3

Summary of the parameter estimates in the Latent Growth Model $(\mathrm{N}=110)$

\begin{tabular}{|c|c|c|c|}
\hline & Estimate & S.E. & $P$ \\
\hline \multicolumn{4}{|l|}{ Regression weights } \\
\hline Intercept & -.174 & .083 & .036 \\
\hline Slope & -.004 & .003 & .159 \\
\hline Condition $\mathrm{x}$ Intercept & .124 & .117 & .291 \\
\hline Condition $\times$ Slope & .001 & .004 & .884 \\
\hline \multicolumn{4}{|l|}{ Variances } \\
\hline D1 & .104 & .012 & $* * *$ \\
\hline D2 & .104 & .012 & $* * *$ \\
\hline D3 & .104 & .012 & $* * *$ \\
\hline UO & .309 & .048 & $* * *$ \\
\hline U1 & .000 & & \\
\hline \multicolumn{4}{|l|}{ Covariance } \\
\hline $\mathrm{U} 0 \leftrightarrow \mathrm{UI}$ & .000 & & \\
\hline
\end{tabular}

Note. Both the variance UI and the covariance between $\mathrm{UO}$ and $\mathrm{U} 1$ were fixed at 0 in order to make the model admissible, hence that they do not have a standard error or $\mathrm{p}$-value. Condition = control or experimental group; Intercept = baseline log alcohol use index; Slope = change log alcohol use index. ${ }^{* * *} p<.001$

Based on the delta $\chi^{2}$ test, we decided that the model represented in Figure 2 fits the data relatively well compared with more elaborate models, such as a model with a quadratic effect of time. This model was accepted as fitting the data $\left(\chi^{2}(6)=3.687, p=\right.$ $.719, \mathrm{RMSEA}=.000, \mathrm{CFI}=1.000, \mathrm{TLI}=1.028)$. As indicated in Figure 2, the latent factors intercept and slope are extracted from the alcohol use index data across pre-test, posttest and 6 month follow-up (i.e. from the alcohol use index at 0, 4, 26 weeks). These are used as estimates of baseline functioning (intercept) and the increase or decrease of the alcohol use index over time (slope). The double-headed arrow between intercept and slope indicates the association between the individual level of baseline functioning and the individual change over time. By fixing all paths from the intercept factor to each measurement point to 1 and all paths from the slope factor to each measurement point to $0,4,26$ this model represents linear growth. By introducing condition as a predictor of intercept and slope, the means of these two latent factors are estimated based on group membership. The path from condition to intercept tests if the experimental group differs from the control group in baseline functioning. This is the main effect of condition. The path from condition to slope tests whether individual growth within the experimental group differs from individual growth in the control group. This is the interaction between time and condition we are primarily interested in evaluating.

The regression weights in Table 3 show that there was a non-significant effect of condition on intercept $(B=0.124, p=0.291)$ and on slope $(B=0.001, p=0.884)$. This means that the two treatment conditions did not significantly differ with respect to baseline value and change of the alcohol use index over time. That is, there was no effect of condition on baseline functioning, and no interaction between time and condition. The main effect of time was also non-significant $(B=-0.004, p=0.159)$. 


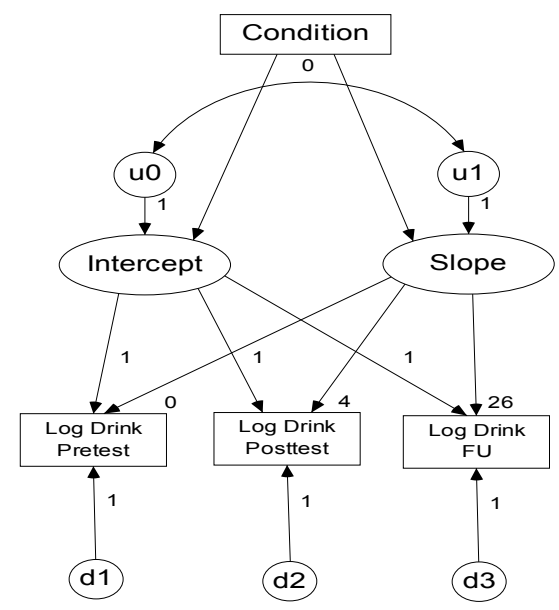

Figure 2. Latent growth model for the log alcohol use index. The labels mark path coefficients that were constrained across assessment points. Condition = control or experimental group; Intercept $=$ baseline log alcohol use index; Slope = change log alcohol use index; Log Drink Pre-test = log alcohol use index score at pre-test; Log Drink Post-test $=\log$ alcohol use index score at post-test; Log Drink FU = log alcohol use index score at six month follow-up.

The initial level of alcohol use index was significantly different from zero $(B=-0.174, p=$ 0.036).

The variance of the latent variables $u 0$ was significantly different from zero (var (v0) $=0.309, p<0.001$ ), meaning that there was between-individual variability in the initial level of alcohol use index. Both the variance UI and the covariance between UO and UI were fixed at 0 in order to make the model admissible (these values were estimated negative if not fixed). The variances of the latent variables $d \mathrm{l}-\mathrm{d} 3 \mathrm{w}$ were estimated equal to each other; the model were each variance was estimated individually did not lead to a significant increase in model fit compared to this model. The variances of the latent variables d l-d3 were all significantly different from zero (var (d l-d3) $=.104, p<$ .001 ), meaning that there were other factors that contribute to the variability in the alcohol use index scores at the first three time points than those used in the model.

\section{DISCUSSION}

This study investigated the influence of motivational interviewing (MI) on explicit and implicit alcohol-related cognitions and alcohol use in at-risk adolescents. It was hypothesized that in the experimental group motivation to change and negative expectancies would increase compared to the control group. Second, it was hypothesized that the experimental group would decrease their drinking compared with the control group. Third, we did not expect that the intervention would directly affect implicit 
alcohol-related cognitions, but rather that a reduction in drinking would be achieved through a moderating effect of an increased motivation to change. The first hypothesis was not confirmed; readiness to change and negative expectancies were not differentially affected by the MI. There was also no group difference in drinking at post-test and follow-up. Given these null findings, the moderation hypothesis could not be tested.

In line with dual process models of addiction, both explicit and implicit alcoholrelated cognitions were correlated with current drinking behavior. This is consistent with previous research on implicit and explicit cognitive processes in at-risk adolescent populations (e.g. Ames et al., 2007; Thush, Wiers, Ames et al., 2007). Additionally, the results indicated that adolescents who drank more heavily and experienced more alcohol-related problems had a stronger inclination to change their current drinking behavior. However, the results regarding the effectiveness of the intervention did not yield support for any differential short term or long term effects of the intervention on drinking behavior. A possible explanation might be that there was no increase in readiness to change in the experimental group. Although it is assumed that $\mathrm{Ml}$ increases motivation to change (Miller \& Rollnick, 2002) and the quality of intervention was rated positively, readiness to change was not effected by the intervention. This raises some questions regarding the effectiveness of $\mathrm{MI}$ in some (at-risk) adolescent populations. Although brief MI has been proven to be effective in reducing hazardous drinking in (young) adult populations, this strategy might be less effective in (at-risk) adolescents, which could be explained by the differential response to alcohol in adolescents and (young) adults. During adolescence the positive arousal effects of drinking are being experienced more so than the negative effects (NIAAA, 2005). Therefore, these adolescents might feel they do not have sufficient reason for changing their drinking behavior (Thush, Wiers, Theunissen et al, 2007). Consequently, it would be useful for future research to investigate the developmental variation in intervention response in some more detail.

Even though there were no changes in drinking behavior or readiness to change, some changes in alcohol-related cognitions between pre-test and post-test were found. Based on current dual process models, we did not hypothesize any changes in implicit cognitive processes, given that these processes are generally quite robust and not easy to change with a short intervention (e.g. Fazio \& Towles-Schwen, 1999; Strack \& Deutsch, 2004). Furthermore, there is mixed evidence for the malleability of implicit cognitions over the course of treatment, although very few studies have examined this question to date. Regarding alcohol abuse, one study demonstrated that a cognitive behavioral intervention (an expectancy challenge) primarily affected explicit alcoholrelated cognitions, with minor and unrelated changes in implicit associations (Wiers, Van de Luitgaarden, Van den Wildenberg, \& Smulders, 2005). There is some evidence in other domains of psychopathology, such as spider phobia, that treatment can be effective in changing implicit associations towards spiders (cf. Teachman \& Woody, 2003). On the other hand, there are some methodological concerns about the implicit 
measure used (Thorpe \& Salkovskis, 1997) and the effects of repeated testing on these results (Huidijng \& de Jong, 2007). Although repeated testing certainly might have played a role in the pre- and post-test assessment of implicit cognitions here, this cannot explain the differential effects between the experimental and control group. Another possible explanation for the increase in attentional bias in the experimental group found here could be that the intervention succeeded in putting alcohol "on the agenda". However, given that the participants in the experimental group did not report more readiness to change or a change in drinking behavior, the effect of the supposed agenda setting can be regarded as minor.

The present results should be interpreted with some caution, given some limitations. First, although we screened for schools with a high proportion of at-risk adolescents, we did not use a probability sampling strategy at an individual level and therefore we cannot generalize these results to other at-risk adolescent populations. In addition, we did not have the statistical power to adequately perform a multiple group analysis. It could be that for specific subgroups of at-risk adolescents the results would have been different. Second, there are some limitations regarding the sequence of the measures used in the current study and the measures themselves. Since the Flicker Task, IAT, and the alcohol expectancy, alcohol use, and readiness to change questionnaires were performed in a fixed sequence possibly order effects could have played a role in the current results. However, this fixed sequence was chosen as the optimal procedure to minimize method-related variance in a study focusing on individual differences (cf. Asendorpf, Banse, \& Mücke, 2002). Additionally, some authors question the validity of the IAT, given that IAT results can also be explained by simple recoding based on salience asymmetries (e.g. Rothermund \& Wentura, 2004; De Houwer, 2001). However, in alcohol-IATs similar to the ones used here, these salience asymmetries could only partly explain the results found (Houben \& Wiers, 2006). Moreover, since we mixed conceptual and perceptual task characteristics within one test, we may have been able to provide some defense against possible recoding strategies given the fact that not just one simple recoding strategy can be used. Since participants do not react just to one dimension of interest, the task may be less prone to response strategies the participants might want to employ (cf. De Houwer, 2003; Rinck \& Becker, 2007).

Finally, the influence of motivation on implicit alcohol-related cognitions might not be seen separately from the capacity to restrain oneself. Even if someone is very motivated and has a specific goal in mind, one still needs to be able to apply the executive control needed to achieve this goal (Feldman-Barrett et al., 2004; Wiers et al, 2007). Indeed, the concept of motivation does not replace the importance of the capacity to restrain oneself (Towles-Schwen \& Fazio, 2006), just as the concept of executive functioning does not replace the importance of motivation and goals (Feldman-Barrett et al., 2004; Wiers et al, 2007). Moreover, there is evidence that these two moderating forces influence each other as well. Fadardi and Cox (in Wiers, Cox et al., 2006) have shown that motivation to change may be increased by enhancing the 
ability to control oneself. This indicates that these two moderating influences on implicit processes may not be seen separately and possibly have to be studied simultaneously in the field of addictive behaviors.

Summarizing, although these limitations must be taken into consideration when interpreting the present largely null findings, we believe that this study does provide some useful information. First, in line with previous research both implicit and explicit alcohol-related cognitions were correlated with current alcohol use. Therefore, it seems important that both explicit and implicit alcohol-related cognitions are taken into account when intervention methods are being developed. Furthermore, although we did not find that the current brief Ml intervention influenced motivation to change or drinking behavior, $\mathrm{Ml}$ is assumed to affect readiness to change and this in turn is strongly connected to behavior change (Miller \& Rollnick, 2002). Our findings raise some questions regarding the use of a brief $\mathrm{Ml}$ in this particular at-risk adolescent population and the mechanisms through which $\mathrm{Ml}$ is effective. Future experimental research regarding the influence of $\mathrm{Ml}$ on alcohol-related cognitions can possibly shed some light on these issues. 
APPENDIX A

\section{IAT picture stimuli}

Alcohol

Label: alcohol photo
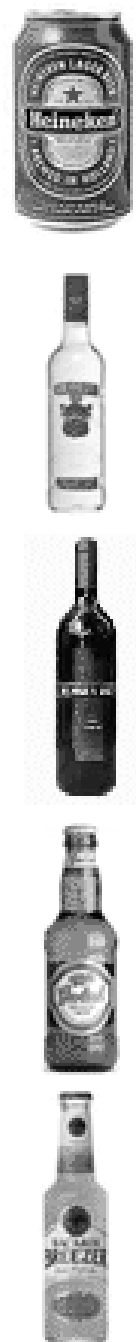

stimuli Neutral stimuli

Label:soda photo
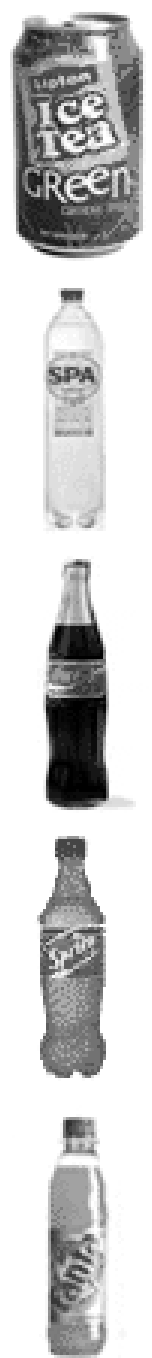


\section{APPENDIX B}

\section{IAT attribute stimuli}

(translated from Dutch)

\begin{tabular}{|c|c|}
\hline Positive Arousal stimuli & $\begin{array}{l}\text { Neutral stimuli } \\
\text { (neutral word) }\end{array}$ \\
\hline (active word) & (neutral word) \\
\hline excited & historical \\
\hline energetic & totally \\
\hline sexy & steep \\
\hline lively & wide \\
\hline wild & common \\
\hline Positive Sedation stimuli & Neutral stimuli \\
\hline (relaxed word) & (neutral word) \\
\hline relaxed & normal \\
\hline calm & blue \\
\hline chill & flat \\
\hline tranquil & figurative \\
\hline dreamy & digital \\
\hline Negative stimuli & Neutral stimuli \\
\hline (miserable word) & (neutral word) \\
\hline sad & daily \\
\hline unhappy & square \\
\hline sick & narrow \\
\hline nauseous & compact \\
\hline miserable & literal \\
\hline
\end{tabular}


APPENDIX C

Flicker picture stimuli

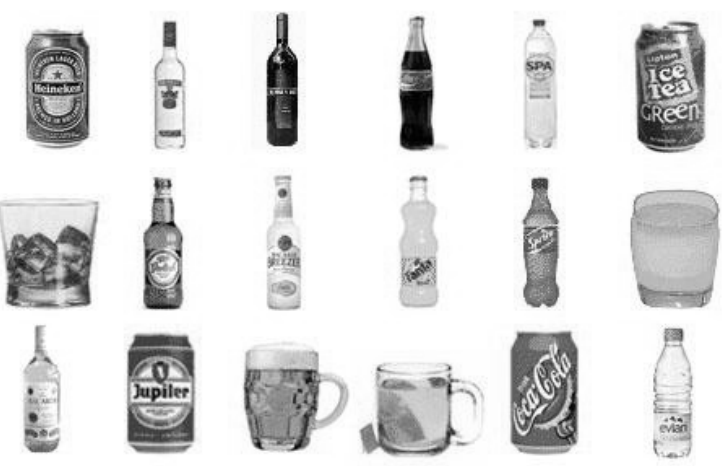

XXXXXXXXXXXXXXXXXXXX XXXXXXXXXXXXXXXXXXX XXXXXXXXXXXXXXXXXXX XXXXXXXXXXXXXXXXXXX XXXXXXXXXXXXXXXXXXX XXXXXXXXXXXXXXXXXXX XXXXXXXXXXXXXXXXXX XXXXXXXXXXXXXXXXXX XXXXXXXXXXXXXXXXXX XXXXXXXXXXXXXXXXXX

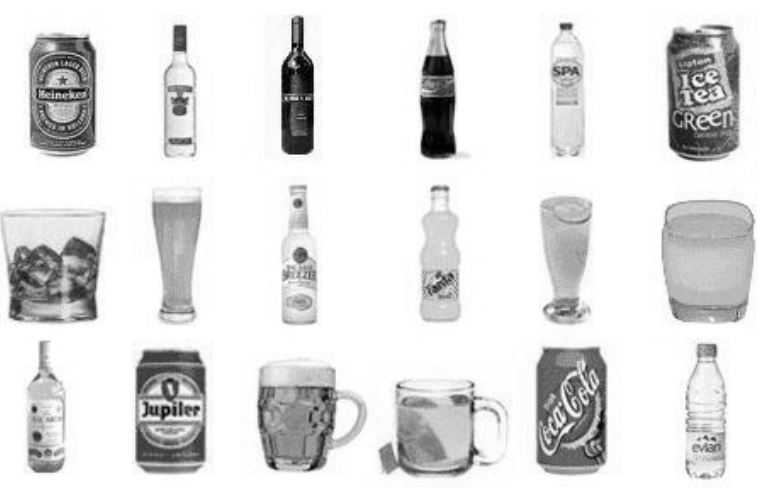





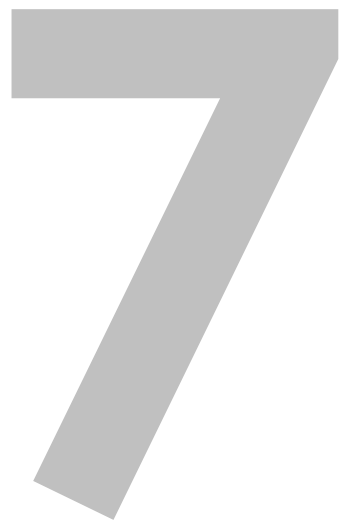

General Discussion

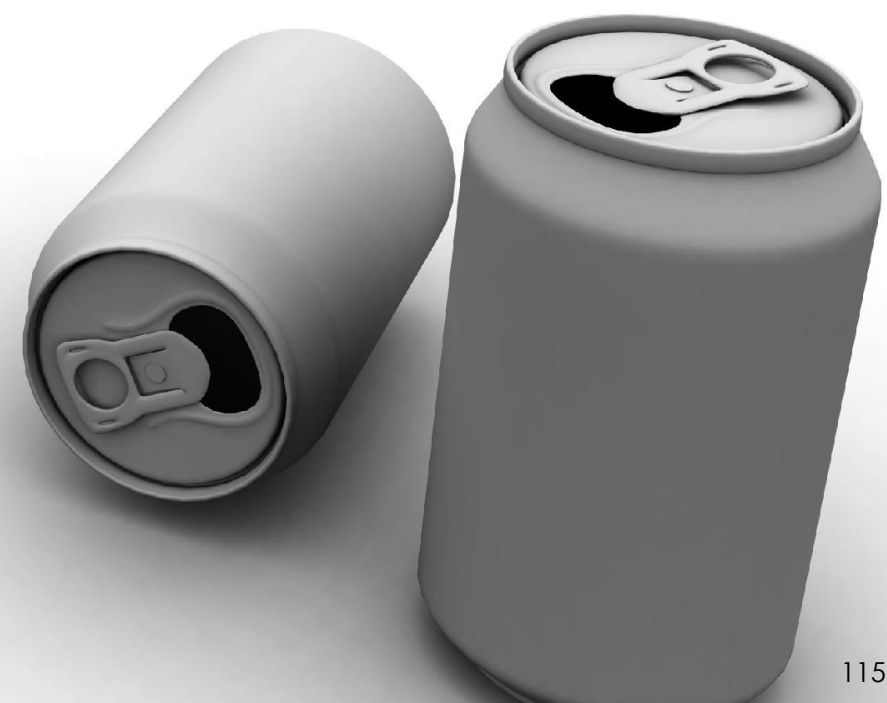




\section{OVERVIEW OF THE MAIN RESULTS}

The central focus of this thesis was to investigate the influence of explicit and implicit cognitive processes on adolescent alcohol use and to test the effectiveness of early intervention within this sample. Based on dual process models of addiction and prior research (e.g. Stacy, Ames \& Knowlton, 2004; Wiers et al, 2007), it was assumed that both implicit and explicit cognitive processes influence (early) adolescent drinking behavior. The relationship between alcohol-related cognition and adolescent alcohol use was assumed to be moderated by other factors, such as executive functioning and motivation. Specifically, if the person in question is motivated enough and has a adequate level of executive control, explicit reflective cognitive processes were expected to moderate the influence of implicit appetitive cognitive processes on drinking behavior. Consequently, intervening at a motivational level and/or changing deliberate explicit reasons to drink should lead to a change in drinking behavior. This thesis investigated these assumptions that followed from the dual process perspective on addiction.

In Chapter 2, the seven session intervention, which primarily aimed at changing explicit alcohol-related cognition, did not succeed in moderating adolescent alcohol use (Chapter 2). One explanation why the intervention did not succeed in changing drinking behavior might be that the influence of implicit alcohol-related cognitive processes was not considered in development of the program. Indeed, three studies confirmed that both implicit and explicit cognitive processes predicted (early) adolescents drinking behavior independently (Chapter 3, 4 and 5). Where word association tasks are to be used when one wants to establish the influence of implicit processes on adolescent drinking behavior, it is better to use the Implicit Association Task (IAT) when one wants to compare the content of these implicit alcohol-related processes to more explicit alcohol-related processes (Chapter 4). With different versions of the IAT, it was shown that primarily positive arousal associations positively predicted or correlated with adolescent drinking behavior (Chapter 3, 5, and 6), whereas mainly explicit negative alcohol expectancies negatively predicted or correlated with adolescents alcohol use (Chapter 3, 4, and 6). In addition, results clearly indicated that implicit and explicit alcohol-related cognition influenced behavior through different pathways (Chapter 5). Adolescents with a below average level of working memory capacity let their drinking behavior be guided by more implicit appetitive alcohol-related processes. Conversely, more explicit alcohol-related processes determined drinking behavior in adolescents with an above average level of working memory capacity. This shows that executive functioning acts as a moderator in the relationship between alcohol-related cognition and adolescent alcohol use. Whether motivation acts as a moderator remains to be seen. Given that the single session Ml intervention did not succeed in enhancing motivation to change or moderating adolescent alcohol use, it could not be determined whether enhanced motivation leads to moderating the influence of more 
implicit appetitive alcohol-related processes on subsequent drinking behavior (Chapter 6).

These main findings will be discussed in relation to dual process models of addiction (Stacy et al, 2004; Wiers et al, 2007) Furthermore, the findings will be related to research on implicit and explicit cognitive processes, interventions and adolescent development. Finally, the implications for future experimental and clinical research on adolescent alcohol use are discussed

\section{ALCOHOL USE \& COGNITIVE PROCESSES}

Dual process models of addiction predict that the influence of implicit appetitive cognitive processes on subsequent substance use might be restrained by more explicit reflective cognitive processes (Stacy et al., 2004; Wiers et al, 2007). Indeed, neurobiological addiction research has implicated that the prefrontal cortex and associated areas are not only involved in more reflective decision making, but also in the moderation of impulses (e.g. Bechara, 2005; Kalivas \& Volkow, 2005; Wilson, Sayette \& Fiez, 2004). However, whether these explicit reflective processes can moderate the influence of implicit associative processes depends on the individual level of executive functioning and amount of motivation. The immediate positive-arousal effects of alcohol act as relatively automatic associations that guide drinking behavior, while more distal negative effects of drinking need more time, effort and reflection to restrain these automatic influences and affect behavior (Deutsch \& Strack, 2006; Wiers et al, 2007).

The results in this thesis are generally in line with these recent dual process models of addiction (Stacy et al, 2004; Wiers et al, 2007). Both implicit and explicit cognitive processes predicted adolescents drinking behavior in Chapter 3, 4, and 5. Overall, especially primarily positive arousal associations positively predicted or correlated with adolescent drinking behavior (Chapter 3, 5, and 6), whereas mainly explicit negative alcohol expectancies negatively predicted or correlated with adolescents alcohol use (Chapter 3, 4, and 6). Furthermore, there is additional evidence in Chapter 5 that these implicit cognitive processes influence behavior through a different pathway than explicit cognitive processes. The results in Chapter 5 show that the influence of these implicit appetitive cognitive processes is indeed moderated by executive functioning. Unfortunately, it could not be determined whether explicit reflective cognitive processes moderate the influence of implicit appetitive cognitive processes and that motivation possibly act as a moderating variable in this relationship (Chapter 6).

\section{Explicit Alcohol-Related Cognition}

Negative alcohol expectancies were the strongest predictors within the group of alcohol expectancies of adolescent drinking behavior (Chapter 3 and 4). Adolescents 
who have stronger negative alcohol expectancies tend to drink less. Negative alcohol expectancies can thus be regarded as a protective factor. Dual process models, such as the general cognitive model by Strack and Deustch (2004) and the addictionrelated model by Wiers et al (2007), expected exactly this. While the immediate positive-arousal effects of drinking are stored as associations that guide behavior implicitly, more distal and often negative effects of drinking alcohol require a more explicit reflective processing mode to influence subsequent behavior. Given these results and the assumptions from a dual process perspective, it might be valuable to consider these negative alcohol expectancies as important factors that can prevent hazardous adolescent alcohol use. Unfortunately, prior studies on adolescent alcohol expectancies have focused primarily on positive arousal expectancies, both in measurement and how to challenge these expectancies (Dunn \& Goldman, 1998, 2000; Darkes \& Goldman, 1993; 1998; for critical review see Jones, Corbin \& Fromme, 2001). Yet, in adult addiction research it has already been shown that negative alcohol expectancies might be the key to more successful treatment outcomes (Jones \& McMahon, 1996). Even so, it must be said that the negative alcohol expectancies adult alcoholics hold are probably different from the negative alcohol expectancies these adolescent hold, given the difference in duration of direct experience with alcohol (mis)use. Negative alcohol expectancies due to prolonged alcohol (mis)use or dependency consist of more long term consequences of alcohol abuse, such as losing one's job and marital or family-related stress (Jones \& McMahon, 1996). In sum, negative alcohol expectancies can be regarded as important protective factors against hazardous adolescent drinking. Nonetheless, these adolescent expectancies have to be seen as different beliefs about drinking outcomes than adult alcohol expectancies.

Indeed, alcohol expectancies tend to change with age and drinking experience. Older and heavier drinking adolescents tend to hold stronger positive and arousal expectancies and weaker negative alcohol expectancies than younger and lighter drinking or abstaining adolescents (Chapter 3). This is generally in line with prior research on the development of adolescent alcohol expectancies (Cameron, Stritzke \& Durking , 2003; Dunn \& Goldman, 1998, 2000; Wiers, Hoogeveen, Sergeant \& Gunning, 1997). However it might well be that for adolescents, who become or remain problematic drinkers into early adulthood, explicit negative alcohol expectancies or implicit negative associations with alcohol become increasingly apparent (e.g. Jones \& McMahon, 1996; Wiers, van Woerden, Smulders \& De Jong, 2002). Thus, adolescents hold stronger positive and arousal alcohol expectancies and weaker negative alcohol expectancies the older and more experienced with alcohol they become. However, when alcohol use increases beyond a certain threshold, alcohol-related problems and thus negative alcohol-related cognition may in turn positively predict alcohol (ab)use as well. 
Table 1.

Internal consistencies of the different indirect measures

\begin{tabular}{l|l|l|}
\hline Chapter 3 & ST-IAT & .43 to .52 \\
\hline Chapter 4 & Word Association Task & .29 \\
\hline & EAST & .07 to .62 \\
\hline & Unipolar IAT & .52 to .59 \\
\hline Chapter 5 & Unipolar IAT & .52 to .59 \\
\hline Chapter 6 & Bipolar IAT & .57 to .64 \\
\hline
\end{tabular}

Note. The internal consistency of each task is estimated by calculating Cronbach's alpha: in the IAT by correlating practice and test items, in the EAST by correlating two halves of the alcohol words in the test block and in the word association tasks by correlating the three separate subtasks.

\section{Implicit Alcohol-Related Cognition}

Implicit cognitive processes uniquely influence drinking behavior in adolescents (Chapter 3, 4 and 5). This is in line with other studies that have shown both implicit and explicit alcohol-related cognitions to predict a unique part of the variance in current and prospective alcohol use (e.g. Jajodia \& Earleywine, 2003; Stacy, 1997; Wiers et al., 2002). Looking at which implicit alcohol associations influence drinking behavior, one can conclude that mostly positive arousal associations positively predicted or correlated with drinking behavior (see Chapter 3, 5, and 6). Again, this is in line with prior addiction research on adult alcohol use (for overview see for example Houben, 2007), but also matches the assumptions made from a dual process perspective. The immediate positive arousal effects of drinking alcohol are stored as memory associations that guide drinking behavior implicitly (Deutsch \& Strack, 2006, Wiers et al, 2007).

With direct experience with alcohol and increasing age, these implicit associations with alcohol tend to change. Before adolescents commence with drinking, their explicit association with alcohol is primarily negative, although they tend to become more ambivalent with increasing age due to an implicit positive association with alcohol (O'Connor, Fite, Nowlin, \& Colder, 2007). Thus, already before adolescents have any direct experience with alcohol, these adolescents seem to become more susceptible to commence with drinking alcohol. Once adolescents initiate drinking and thus have direct experience with drinking alcohol, their implicit association with alcohol becomes increasingly more positive, more arousal-related and less negative. This is sometimes already apparent in very early stages of alcohol consumption (Chapter 3). Thus, like on a more explicit level, adolescents have stronger implicit positive arousal associations with alcohol when they become older and have more experience with drinking.

Implicit alcohol-related cognition can be measured using various indirect measures derived from different research paradigms. The field of research on indirect measures sometimes resembled a field where newly sown measurement tools grow and flourish, until there is such a large variety, it is difficult to oversee which measure most suitable. The question which measure is best in assessing implicit alcohol-related cognition can be answered by comparing how well these measures predict behavior (standardized 
regression coefficients) and how well they measure one underlying concept (internal consistency).

The internal consistencies of the different IATs in Chapter 3, 4, 5 and 6 do not vary that much (see Table 1). However, when one compares the reliability of the IATs with word association tasks and the EAST, it is clear that the IAT is superior in this respect to these other tasks (see Table 1). These finding are generally in line with research that compared the IAT with different indirect measure (e.g. De Houwer \& De Bruycker, 2007). The question remains how much value we can attach to the internal consistency. On the one hand, a participant should react in a similar way to different stimuli that constitute one category (e.g. 'beer' and 'wine' should elicit a similar response given that they both belong to the category 'alcohol'). On the other hand, we include different stimuli or exemplars, given that we expect that participants have a stronger association with one stimulus than with another. The value we assign to the internal consistency of a measure should depend to what extent this measure was designed to measure the association of a heterogeneous category (e.g. 'alcohol'). For instance, one could argue that in the case of the IAT, internal consistency is an informative criterion for the quality of the task given its categorical nature. However, in the case of word association tasks, the usefulness of internal consistency as a criterion for the quality of this task is deflated, given that these tasks are designed to target very specific associations.

When the predictive value of these indirect tasks is compared by looking at the standardized regression coefficients, word association tasks seem best in predicting behavior (see e.g. Chapter 4). This is also confirmed by a related study by Ames et al. (2007). However, as was pointed out in Chapter 4, there are other theoretical reasons one might wants to use reaction-time based measures of associations, such as the IAT. If the aim is to predict behavior, word association tasks seem more suitable than any other task. If the aim is to investigate the content of these implicit cognitive processes compared to more explicit cognitive processes, a version of the IAT (or another indirect task) is maybe more suitable. Thus, comparing these tasks on methodological properties helps to determine which measure is most suitable. However, a variety of tasks and procedures is also needed to be able to answer different research questions.

\section{Executive Functioning}

Executive functioning was shown to moderate the relationship between alcoholrelated cognitive processes and adolescents alcohol use. Adolescents with a below average level of executive functioning tended to let their drinking behavior be guided by more implicit alcohol-related processes, whereas adolescents with an above average level of executive functioning determined tended to let their behavior be determined by more explicit alcohol-related processes (see Chapter 5). These results are in line with prior research on moderating effects of executive functions on implicit cognitive processes. Implicit cognition has been shown to be predictive of behavior in 
individuals with relatively low working memory capacity or attentional control (Grenard et al, in press; Payne, 2005). In addition, our findings are consistent with the findings of Tapert, McCarthy, Aarons, Schweinsburg and Brown (2003) that explicit positive alcohol expectancies predicted alcohol use in substance use disorder adolescents with good verbal skills, whereas they did not in substance use disorder adolescents with poor verbal skills. This indicates that alcohol expectancies may require deep processing in order for them to affect decision making and thus drinking.

This implicates that in order to change behavior, different strategies might be useful for different groups of adolescents. For example, at-risk adolescents with a below average level of executive functioning might benefit from interventions that attempt increasing their capacity to regulate impulses and to apply executive control. On the other hand, this also shows that there is a subgroup of adolescents with a high level of executive functioning that are specifically guided by explicit cognitive processes. This coincides with the idea that not all adolescents engage in risky behavior merely because there are unable to apply cognitive control. These adolescents might benefit from interventions that aim to enhance negative alcohol expectancies.

\section{Motivation}

Recently, it has been suggested that adolescents might engage in risky behavior, aware of the possible costs involved, to confirm or enhance their social status (Allen, Porter, McFarland, Marsh, \& McElhaney, 2005). Especially for this group of adolescents, it seems plausible to focus more on the underlying motivation or deliberate reasons to drink in stead of focusing primarily on executive control. The importance of having adaptive motivational structures in the context of changing one's tendencies toward more addictive behaviors has been demonstrated by a large body of research (Cox \& Klinger, 2002; Milner \& Rollnick, 2002). Furthermore, prior studies in other research domains have shown that the influence of implicit processes on behavior increases when motivation not to act on these automatic associations is low (e.g. Olson \& Fazio, 2004). Although motivation was expected to moderate the relationship between alcoholrelated cognitive processes and adolescents alcohol use, we could not test this hypothesis directly given that the intervention did not succeed in increasing motivation to change (see Chapter 6). However, other research has shown that concept of motivation does not replace the importance of the capacity to restrain oneself (TowlesSchwen \& Fazio, 2006). Thus, the influence of motivation on implicit cognitive processes might not be seen separately from the capacity to restrain oneself. Even if someone is very motivated and has a specific goal in mind, one still needs to be able to apply the executive control needed to achieve this goal (Feldman-Barrett, Tugade \& Engle, 2004; Wiers et al, 2007). 


\section{ALCOHOL USE \& INTERVENTION}

The large number of adolescents, that engages in hazardous drinking behavior in spite of primary prevention (in our samples sometimes up to $77 \%$ engaged in binge drinking), illustrates the need for secondary or targeted intervention. However, it is debatable whether longer lasting interventions show superior effectiveness compared to brief interventions in non-treatment seeking populations (e.g. Moyer, Finney, Swearingen, \& Vergun, 2002). By comparing Chapter 2 and Chapter 6 we could not find evidence that a relatively longer duration of the intervention leads to better outcomes. This is consistent with prior research which has shown that brief intervention can be just as effective (or ineffective) as longer preventive intervention programs (e.g. Bear et al., 1992; Project Match Research Group, 1997).

Additionally, it is the question whether interventions that focus primarily on changing explicit alcohol-related cognition are effective in changing behavior. For instance, in Chapter 2 the seven session intervention succeeded in changing explicit alcohol expectancies, however, this had no effect on adolescent drinking behavior. The fact that we did not observe the expected change in the subsequent outcome behavior, might reflect that targeting these cognitive determinants alone is not enough to elicit meaningful changes in at-risk youth. Although cognitive behavioral skills-based interventions and brief motivational feedback have been proven to be effective in reducing hazardous drinking in (young) adult populations (Larimer \& Cronce, 2002), these strategies might be less effective in at-risk adolescents populations. This reduced effectiveness in these at-risk adolescent populations might be explained by the differential response to alcohol in adolescents and adults. During adolescence not so much the negative effects, but especially the positive arousal effects of drinking are being experienced (NIAAA, 2005). Therefore, these adolescents might feel they do not have sufficient reason for changing their drinking behavior.

Furthermore, these intervention strategies might be less effective in these at-risk adolescent populations, because adolescent drinking behavior might be influenced more by implicit associative processes than explicit reflective processes, such as alcohol outcome expectancies. In other domains of psychopathology, for instance, research has shown that when only explicit cognition changes due to treatment but implicit cognition remains the same, that these implicit cognitive processes predict the return of fear (Huijding \& De Jong, 2007; Teachman \& Woody, 2003). However, it remains to be seen whether interventions that focus primarily on changing implicit alcohol-related cognition are effective in changing behavior. Experimental interventions, such as attentional retraining, are effective in changing implicit cognition but do not always lead to behavior change (Wiers, Cox et al., 2006). Thus, this again indicates that merely influence one of both processes is not enough.

However, part of the problem in testing the effectiveness of the interventions in Chapter 2 and 6 could have been that, although the initial idea was to sample high risk 
adolescent drinkers, we also included light drinking adolescents. Teaching adolescents to drink in a safe way is maybe not such a good idea when these adolescents are not already drinking regularly. For example, research by van der Vorst, Engels, Meeus and Deković (2006) showed that parents who adopt the more lenient strategy of teaching their children how to drink in a responsible way at home actually might worsen the problem. Once adolescents start drinking, they increase the frequency of drinking over the years. Setting norms and applying strict rules in the case of young abstaining adolescents seems to be a more fruitful approach: adolescents of parents who apply this method drink significantly less that their peers who have more lenient parents.

Moreover, other factors need to be considered as well. For example, adolescents tend to exhibit and prefer more risky behavior in the presence of peers than when alone compared to adults (Gardner \& Steinberg, 2005). This could be an alternative explanation for the results found in Chapter 6 , given that the adolescents were tested in small groups of four, but the intervention was performed alone. It could well be that in small peer groups an individual's positive-arousal alcohol expectancies and associations are more activated and accessible from memory than when in a situation by oneself. This might have reduced the effectiveness of the brief Ml intervention.

\section{ADOLESCENTS AT RISK? FUTURE DIRECTIONS}

Adolescence is not only a developmental period in which body and brain mature, but it is also a period which offers other special opportunities as well as vulnerabilities (Dahl, 2004). Adolescents are not merely incapable of making rationally sound decisions, but under certain conditions of high arousal cross-talk between the emotional and cognitive control system is impaired (Steinberg, 2007). This might be reflected in the fact that both explicit and implicit cognitive processes play an important role in the early development of addictive behaviors in adolescence (e.g. Chassin, Presson, Rose, Sherman \& Prost, 2002; Stacy, Ames, Sussman, \& Dent, 2006; Stacy, 1997; see Chapter 3, 4, 5 and 6). For instance, under circumstances of high arousal (e.g. being at a party), both negative alcohol expectancies and implicit positive arousal associations with alcohol may influence adolescent drinking behavior. These processes seem to influence behavior through separate pathways, given that they predict a unique part of the variance in adolescent drinking (Chapter 3 and 4) and influence behavior differently depending on the level of executive functioning (Chapter 5). However, it remains to be seen whether under these circumstances of high arousal, the individual is able to restrain the influence of implicit cognitive processes. One has to be highly motivated and have a high level of executive control to shift back and forth from automatically activated appetitive associations with alcohol to less salient deliberate reasons to refrain from drinking. In addition to investigating these moderation hypotheses in more detail, it is also imperative to determine the relative strength of both implicit and explicit processes 
on drinking behavior and to examine whether these two processes influence each other bidirectionally (Gawronski \& Bodenhausen, 2006). At this point, for instance, we do not know whether and how implicit cognitive processes might influence more explicit cognitive processes.

Furthermore, it is still unclear how these implicit associations are formed and how to change or moderate the influence of these associations. So far, only few scientific attempts have been made to investigate the development of implicit alcohol-related cognitions in the early stages towards alcohol consumption (O'Connor, Fite, Nowlin, \& Colder, 2007; Chapter 3). At this point experimental research is lacking which indicates the factors involved in the development of these implicit associations with alcohol. For instance, the level of executive functioning and amount of motivation may not only be factors that determine how much impact implicit associations have on drinking behavior, but may also determine the formation of these implicit associations. For instance, both negative motivational mood state and poor executive functioning are characterized by diminished top-down processing (e.g. Schwartz, 2000; Miyake, 200). It has been suggested that associative learning might profit from the absence of top down processes (e.g. De Houwer, Baeyens \& Field, 2005). In other words, children with a relatively low level of executive functioning or in a negative motivation mood state might more easily build alcohol-related associations. Future research has to investigate how and under what circumstances these implicit alcohol-related associations are formed (in longitudinal and more experimental design).

Additionally, it is important that we obtain insight how to change or moderate the influence of these implicit alcohol-related associations. In Chapter 5, it is implicated that different intervention methods might be effective for different subgroups of at-risk adolescents. For example, at-risk adolescents with below average working memory capacity might benefit from interventions that attempt increasing their capacity to regulate impulses and to apply executive control. Indeed, research has shown that executive functions and the associated brain regions are not fixed processes or structures but are characterized by their plasticity and can be modified through feedback and training (e.g. Erickson et al., 2007; Olesen, Westerberg \& Klingberg, 2004). There is some evidence that these kinds of training procedures are an effective direct intervention for children diagnosed with ADHD (Klingberg, et al., 2005; Shalev, Tsal \& Mevorach, 2007). Future experimental research has to indicate whether these kinds of training are effective in restoring executive control in other clinical and non-clinical populations of different ages. Additionally, it has to be investigated in a clinical setting whether these training methods indeed have a moderating influence on the impact of implicit cognitive processes on subsequent drinking behavior.

Conversely, at-risk adolescents with good working memory capacity might benefit more from interventions that focus on enhancing negative alcohol expectancies. Given that this subgroup of adolescents seems to drink for more deliberate reasons, it seems logical to intervene on the level of more explicit alcohol-related processes. 
Certainly, research has shown that negative alcohol expectancies can function as a protective factor (see Chapter 3). However, it remains to be seen if merely enhancing negative alcohol expectancies will be enough to moderate adolescent alcohol use, especially given the fact that the positive arousal effects of alcohol are experienced more intensely than the negative effects (NIAAA, 2005).

Likewise, following the dual process perspective on addictive behaviors (e.g. Stacy et al., 2004; Wiers et al, 2007), targeted intervention programs could benefit from behavioral interventions that strengthen motivation to control. Although in Chapter 6 there is little evidence for the effectiveness of $\mathrm{Ml}$ in (at-risk) adolescents, there are reasons to believe that motivational interventions might still be a promising approach in at-risk non-treatment seeking populations (Grenard et al., 2007; Moyer, Finney, Swearingen, \& Vergun, 2002). At this point there is little research yet on the role of motivation on explicit and implicit alcohol-related processes that influence drinking behavior. Given our disappointing results in Chapter 6, we might want to consider other ways to investigate motivation experimentally as a moderating factor in dual process models. In addition, we might need other ways to translate this research into intervention programs.

One possibility is to combine both increasing motivation and executive control in one intervention. As has been pointed out previously, the concept of motivation does not replace the importance of the capacity to restrain oneself (Towles-Schwen \& Fazio, 2006), just as the concept of executive functioning does not replace the importance of motivation and goals (Feldman-Barrett et al., 2004; Wiers et al, 2007). Moreover, there is evidence that these two moderating forces influence each other as well. Fadardi and Cox (in Wiers, Cox et al., 2006) have shown that motivation to change may be increased by enhancing the ability to control oneself. This indicates that these two moderating influences on implicit processes may not be seen separately and possibly have to be studied simultaneously in the field of addictive behaviors.

In sum, adolescence is a period with an increased vulnerability to engage in risky behaviors, such as hazardous drinking. However, research has provided new insights into the cognitive processes that might be fueling adolescents drinking behavior. Both implicit appetitive alcohol-related processes and explicit reflective alcohol-related processes influence adolescent drinking behavior. The immediate positive-arousal effects of alcohol seem to act as relatively automatic associations that guide drinking behavior, while more distal negative effects of drinking appear to need more time, effort and reflection to affect behavior. Executive functioning moderates these relationships between alcohol-related cognition and adolescent alcohol use. Whether specifically these explicit reflective processes moderate the influence of implicit associative processes, when the individual's level of executive functioning and amount of motivation is sufficient, needs further investigation. Some of the related questions raised here, implicate the need to investigate some of the claims of these dual process models of addiction in more detail and the implications for developing interventions. 



\section{Summary}

Adolescence is not only a developmental period in which body and brain mature, but it is also a period which offers other special opportunities as well as vulnerabilities (Dahl, 2004). Adolescents are not merely incapable of making rational decisions, but under certain conditions of high arousal cross-talk between the emotional and cognitive control system appears to be impaired (Steinberg, 2007). Hazardous adolescent alcohol use can be seen as a good example of impaired communication between these different systems or processes. For instance, under circumstances of high arousal (e.g. being at a party), both explicit negative alcohol expectancies and implicit positive arousal associations with alcohol may influence adolescent drinking behavior simultaneously. Where implicit cognitive processes represent more automatic associative motivational processes, explicit cognitive processes are related to more deliberate thought processes (Greenwald \& Banaji, 1995; Kahneman, 2003; Strack \& Deutsch, 2004).

The central focus of this thesis was to investigate the influence of explicit and implicit cognitive processes on adolescent alcohol use and to test the effectiveness of early intervention within this sample. Based on dual process models of addiction and prior research (see e.g. Stacy, Ames \& Knowlton, 2004; Wiers et al, 2007), it was assumed that both implicit and explicit cognitive processes influence (early) adolescent drinking behavior. The relationship between alcohol-related cognition and adolescent alcohol use was assumed to be moderated by other factors, such as executive functioning and motivation. Specifically, it was hypothesized that explicit cognitive processes moderated the influence of implicit cognitive processes when a person is motivated enough and has the opportunity to do so. Consequently, intervening at a motivational level and/or changing deliberate explicit reasons to drink should lead to a change in drinking behavior. The studies presented in this thesis investigated these assumptions that followed from the dual process perspective on addiction.

Chapter 2 described a study in which the effectiveness of a targeted intervention program aimed at changing explicit alcohol-related cognition in at-risk adolescents was investigated in a randomized clinical trial. This program combined intervention methods which have been proven effective in reducing drinking in young adults, such as an expectancy challenge, cognitive behavioral skill training and brief motivational feedback. Additionally, this intervention contained the new element of discussing biological, cognitive and social risk factors for developing alcohol problems. The intervention was effective in changing several of the targeted cognitive determinants: there was a significant increase the perception of risk factors for developing alcoholrelated problems and a significant decrease of positive alcohol expectancies for a high dose of alcohol in the experimental group compared with the control group. Despite the changes in these cognitive determinants of drinking, the experimental group did 
not show a significant difference in decrease of drinking at post-test compared with the control group. The results did not yield support for any differential long term effects of the intervention. So, although the intervention succeeded in changing explicit alcoholrelated cognition, the subsequent decrease in drinking behavior was not obtained.

The fact that we did not observe the expected change in the subsequent outcome behavior, might reflect that targeting these cognitive determinants alone is not enough to elicit meaningful changes in at-risk youth. Although cognitive behavioral skills-based interventions and brief motivational feedback have been proven to be effective in reducing hazardous drinking in (young) adult populations (Larimer \& Cronce, 2002), these strategies might be less effective in at-risk adolescents populations. This reduced effectiveness in these at-risk adolescent populations might be explained by the differential response to alcohol in adolescents and adults. During adolescence not so much the negative effects, but especially the positive-arousal effects of drinking are being experienced (NIAAA, 2005). Therefore, these adolescents might feel they do not have sufficient reason for changing their drinking behavior. Furthermore, these intervention strategies might be less effective in these at-risk adolescent populations, because adolescents drinking behavior might be influenced to a greater extent by other processes than explicit cognitive processes. It has been suggested that alcohol-induced brain damage in the prefrontal cortex during adolescence might lead to reduced executive functioning (e.g. Crews, Braun, Hoplight, Switzer \& Knapp, 2000). In turn, reduced executive functioning might lead to less deliberate control and more automatically implicitly induced behavior (Payne, 2005).

Therefore, in Chapter 3 it was investigated to what extent these implicit alcoholrelated cognitions predict adolescent binge drinking (drinking five or more standard alcohol servings on one occasion) after one year above and beyond several other variables such as explicit alcohol-related cognitions. The hierarchical regression analyses revealed that overall the indirect measures significantly added to the prediction of binge drinking after one year, whereas the direct measures (as a group) did not. However, the final regression model revealed that explicit negative alcohol expectancies next to grade and implicit arousal associations predicted binge drinking after one year. Additionally, a three-way interaction between grade, gender and negative implicit associations significantly predicted binge drinking after one year. Noteworthy, a two-way interaction between gender and implicit positive associations predicted prospective binge drinking at borderline significance. Thus, both implicit alcohol-related cognitions and explicit alcohol-related cognitions predicted a unique part of the variance in adolescent binge drinking.

Consequently, the role of explicit alcohol expectancies in predicting binge drinking after one year may be different and smaller than previously assumed. First, the hierarchical regression model revealed that overall the direct as a group did not add significantly to the prediction of binge drinking after one year. Additionally, the role of explicit alcohol expectancies may be different than previously assumed, because rather than 
positive or arousal alcohol expectancies (Dunn \& Goldman, 1996; 1998; 2000), negative alcohol expectancies significantly predicted binge drinking after one year in the final regression model. This is in line with the notion that negative expectancies play a vital role in the preventive approach of affecting current and future drinking (Jones, Corbin \& Fromme, 2001). Moreover, the results showed that implicit alcohol associations are important in the early development of drinking. The hierarchical regression model revealed that the indirect measures significantly added to the prediction of binge drinking after one year when controlling for gender, grade and explicit expectancies. This is consistent with previous research on the influence of implicit and explicit alcohol cognitions in older adolescents (Stacy, Ames, Sussman \& Dent, 1996).

Implicit alcohol-related cognition can be measured using various indirect measures derived from different research paradigms. Consequently, in Chapter 4 the question was how three of these indirect measures (the Implicit Association Task, the Extrinsic Affective Simon Task and word association tasks) performed in the prediction of alcohol use in at-risk adolescents. Results showed that the indirect measures predicted unique variance in prospective alcohol use after controlling for the direct measure of alcoholrelated cognitions and background variables. This is in line with previous research on implicit and explicit alcohol-related cognitive processes in young adults and adolescents (e.g. Palfai \& Wood, 2001; Stacy, 1997; Thush \& Wiers, 2007; Wiers, Van Woerden, Smulders \& De Jong, 2002). Additionally, the trimmed model showed that the word association tests were the strongest predictor among the group of indirect measures of alcohol use. Similar findings were reported in a comparison of word association, IAT, and EAST in the prediction of marijuana use among at-risk youth in the U.S. (Ames et al., 2007). Thus, the same pattern of result arises across different countries, languages and substances.

One possible explanation for the current pattern of results is that for instance the IAT measures relative associations between predefined categories. In contrast, word association allows for free competition among associates to be generated in response to a variety of cues. Because these word association tasks do not impose categorical constraints on the individual, they increase the likelihood of tapping into individual differences in underlying motivational associative structures. On the one hand, the reaction time measures seem to be limited in the sense that they place categorical constraints on the activation of associative structures; while on the other hand; these categorical constraints could be helpful when specific hypotheses about the content of alcohol-related associations are being assessed. In sum, both types of indirect measures (word association tasks and reaction time measures) have their own unique strengths for which they can be used in addiction research.

The relationship between alcohol-related cognition and adolescent alcohol use was assumed to be moderated by other factors, such as executive functioning and motivation. Therefore, Chapter 5 described a study in which both the interactions between implicit alcohol-related cognitions and working memory capacity and explicit 
alcohol-related cognitions and working memory capacity were explored in the prediction of alcohol use in at risk adolescents. Results from a hierarchical regression analysis showed that both interactions predicted unique variance in one-month follow-up alcohol use after controlling for the main effects and background variables. Interestingly, the signs of these interactions were in the opposite direction. That is, implicit positive-arousal cognitions predicted one-month follow-up alcohol use more strongly in participants with a below average level of working memory capacity, whereas explicit positive-arousal cognitions predicted one-month follow-up alcohol use more strongly in participants with an above average level of working memory capacity.

These results are in line with prior research on moderating effects of executive functions on implicit cognitive processes. Implicit cognition has been shown to be predictive of behavior in individuals with relatively low working memory capacity or attentional control (Grenard et al, in press; Payne, 2005). In addition, our findings were consistent with the findings of Tapert, McCarthy, Aarons, Schweinsburg and Brown (2003) that explicit positive alcohol expectancies predicted alcohol use in substance use disorder adolescents with good verbal skills, whereas they did not in substance use disorder adolescents with poor verbal skills. Verbal skills are needed for developing internal language-based reasoning skills (Luria, 1961). Both findings indicate that alcohol expectancies may require deep processing in order for them to affect decision making and thus drinking. Finally, our results are in agreement with research that found dissociations between implicit and explicit cognitions. For instance, prior studies have shown that explicit cognitions predicted more deliberative behavior whereas implicit cognition predicted more spontaneous behavior (Asendorpf, Banse \& Mücke, 2002; Perugini, 2005). Our results are also congruent with the idea that implicit and explicit cognitive processes influence behavior through different pathways (e.g. Fazio \& Towles-Schwen, 1999; Kahneman, 2003; Strack \& Deutsch, 2004), which clearly has relevance for addictive behaviors (see Wiers \& Stacy, 2006). Thus, both the interaction between working memory capacity and implicit positive-arousal cognitions and the interaction between working memory capacity and explicit positive-arousal cognitions could be taken into account when prevention and intervention methods are being developed.

This implicates that different intervention methods might be effective for different subgroups of at-risk adolescents. On the one hand, at-risk adolescents with relatively good working memory capacity might benefit from interventions that try to strengthen protective negative alcohol cognitions, such as a motivational interview, which appears feasible in at-risk adolescents (Grenard et al., 2007). On the other hand, at-risk adolescents with relatively poor working memory capacity might benefit from interventions that attempt to directly interfere with their automatic reactions, such as an attentional retraining (e.g. Wiers et al., 2006) or with interventions that aim at increasing their capacity to regulate impulses, such as a working memory training, as has been successfully used in children with ADHD (Klingberg et al., 2005). However, it should be noted that even if an increase in working memory capacity could also be achieved in 
at-risk adolescents, this does not replace the importance of motivation and goals: the adolescent still needs to be motivated or have a goal in mind to apply this executive control (Feldman-Barrett, Tugade \& Engle, 2004; Wiers et al, 2007). Future research is needed to indicate if and which methods can be included as proven effective components in intervention programs.

Hence, in Chapter 6 we investigated what the influence of Motivational Interviewing (MI) was on implicit and explicit alcohol-related cognitions and subsequent alcohol use in at-risk adolescents. From a dual process perspective on addiction (Stacy et al., 2004; Wiers et al, 2007), intervening at a motivational level and/or changing deliberate explicit reasons to drink should lead to a change in drinking behavior. Nevertheless, we did not find that the current brief Ml intervention influenced motivation to change or drinking behavior, although $\mathrm{Ml}$ is widely assumed to affect readiness to change and this in turn is strongly connected to behavior change (Miller \& Rollnick, 2002). Our findings raise some questions regarding the use of a brief $\mathrm{Ml}$ in this particular at-risk adolescent population and the mechanisms through which $\mathrm{Ml}$ is effective. Future experimental research regarding the influence of $\mathrm{MI}$ on alcohol-related cognitions can possibly shed some light on these issues.

However, the concept of motivation does not replace the importance of the capacity to restrain one-self (Towles-Schwen \& Fazio, 2006), just as the concept of executive functioning does not replace the importance of motivation and goals (Feldman-Barrett et al., 2004; Wiers et al, 2007). Moreover, there is evidence that these two moderators influence each other as well. Fadardi and Cox (in Wiers et al., 2006) have shown that motivation to change may be increased by enhancing the ability to control oneself. This indicates that these two moderating influences on implicit processes may not be seen separately and possibly have to be studied simultaneously in the field of addictive behaviors.

Concluding, adolescence is a period with an increased vulnerability to engage in risky behaviors, such as hazardous drinking. Research has provided new insights into the cognitive processes that might be fueling adolescents drinking behavior. Both implicit appetitive alcohol-related processes and explicit reflective alcohol-related processes influence adolescent drinking behavior. The immediate positive-arousal effects of alcohol seem to act as relatively automatic associations that guide drinking behavior, while more distal negative effects of drinking appear to need more time, effort and reflection to affect behavior. Executive functioning moderates these relationships between alcohol-related cognition and adolescent alcohol use. Whether specifically these explicit reflective processes moderate the influence of implicit associative processes, when the individual's level of executive functioning and amount of motivation is sufficient, needs further investigation. Some of the related questions raised here, implicate the need to investigate some of the claims of these dual process models of addiction in more detail and the implications for developing interventions. 



\section{Samenvatting}

De adolescentie is niet alleen een ontwikkelingsperiode waarin het lichaam en de hersenen volgroeien, maar het is ook een periode die andere speciale mogelijkheden en kwetsbaarheden biedt (Dahl, 2004). Adolescenten zijn niet zozeer incapabel om rationale beslissingen te nemen, maar onder bepaalde omstandigheden van verhoogde arousal lijkt de communicatie tussen het emotionele systeem en het cognitieve controle systeem verstoord (Steinberg, 2007). Overmatig alcoholgebruik in de adolescentie kan worden gezien als een goed voorbeeld van een miscommunicatie tussen deze systemen of processen. Bijvoorbeeld onder omstandigheden van verhoogde arousal, zoals het aanwezig zijn op een feestje, kunnen zowel expliciete negatieve alcohol verwachtingen als impliciete positieve arousal associaties met alcohol adolescent drinkgedrag beïnvloeden. Waar impliciete cognitieve processen meer automatische associatieve motivationele processen representeren, zijn expliciete cognitieve processen meer gerelateerd aan doelbewuste denkprocessen (Greenwald \& Banaji, 1995; Kahneman, 2003; Strack \& Deutsch, 2004).

Het voornaamste doel van dit proefschrift was dan ook om de invloed van deze expliciete en impliciete cognitieve processen op adolescent alcoholgebruik te onderzoeken en om de effectiviteit van vroege interventie binnen deze doelgroep te onderzoeken. Op basis van duale proces modellen van verslaving en eerder onderzoek (bijvoorbeeld Stacy, Ames \& Knowlton, 2004; Wiers et al, 2007), werd verondersteld dat zowel impliciete als expliciete cognitieve processen een rol spelen in het bepalen van het drinkgedrag in de (vroege) adolescentie. De relatie tussen alcoholgerelateerde cognitie en adolescent alcoholgebruik werd geacht te worden gemodereerd door andere factoren, zoals executief functioneren en motivatie. Om precies te zijn, werd er verwacht dat expliciete cognitieve processen de invloed van impliciete cognitieve processen kunnen beteugelen, wanneer de persoon gemotiveerd genoeg is en genoeg mogelijkheden heeft om dit te doen. Ingrijpen op het niveau van motivatie en/of doelbewuste expliciete redenen om te drinken zou dus moeten leiden tot een verandering van drinkgedrag. De studies gepresenteerd in dit proefschrift hebben deze assumpties, die volgen vanuit een duale proces modellen van verslaving, onderzocht.

In hoofdstuk 2 werd een studie beschreven, waarin de effectiviteit van een interventie programma, gericht op het veranderen van expliciete cognitieve processen bij risicojongeren, werd onderzocht in een gerandomiseerd klinische trial. Dit interventie programma combineerde interventie methoden, zoals een expectancy challenge, cognitieve gedragstraining en motivationele feedback, die in het verleden effectief zijn gebleken in het reduceren van alcoholgebruik in jong volwassenen. Daarnaast bevatte deze interventie een nieuw element waar de biologische, cognitieve en sociale risicofactoren voor het ontwikkelen van een alcoholprobleem werden besproken. De interventie was effectief in het veranderen van verschillende beoogde cognitieve 
determinanten: er was een significante toename in de perceptie van risicofactoren voor het ontwikkelen van een alcoholprobleem en een significante afname in positieve alcohol verwachtingen voor een hoge dosis alcohol in de experimentele groep in vergelijking tot de controle groep. Echter, ondanks deze veranderingen in de cognitieve determinanten van drinkgedrag, vertoonde de experimentele groep geen significant verschil in de afname van drinken op post-test in vergelijking tot de controle groep. De resultaten laten geen lange termijn effect van de interventie zien. Dus, hoewel de interventie er in slaagde expliciete alcoholgerelateerde cognitie te veranderen, bleef de relatieve afname in drankgebruik ten opzichte van de controle groep vit.

Het feit dat we de verwachte verandering in gedrag niet konden waarnemen, kan te wijten zijn aan dat het focussen op voornamelijk deze cognitieve determinanten niet genoeg is om verandering in gedrag bij risicojongeren te bewerkstelligen. Hoewel cognitieve gedragstraining en motivationele feedback in het verleden effectief zijn gebleken in het terugdringen van overmatig alcoholgebruik in de populatie van jong volwassenen (Larimer \& Cronce, 2002), kunnen deze strategieën minder effectief zijn in een populatie van risicojongeren. De verminderde effectiviteit in deze populatie kan worden verklaard door het feit dat adolescenten een andere reactie op alcohol vertonen dan volwassenen. Adolescenten ervaren niet zozeer de negatieve effecten, maar meer de positieve-arousal effecten van het drinken van alcohol (NIAAA, 2005). Daardoor kan het zo zijn dat adolescenten vinden dan ze niet genoeg reden hebben om hun drinkgedrag te veranderen. Bovendien kunnen deze interventie strategieën minder effectief zijn in deze populatie van risicojongeren, omdat adolescent drinkgedrag mogelijk in grotere mate wordt beïnvloed door andere processen dan door expliciete cognitieve processen. Het wordt bijvoorbeeld verondersteld, dat de schade in de prefrontale cortex door overmatig alcoholgebruik tijdens de adolescentie kan leiden tot verminderd executief functioneren (Crews, Braun, Hoplight, Switzer \& Knapp, 2000). Verminderde executieve functies kunnen op hun beurt leiden tot minder doelbewuste controle en meer automatisch geactiveerd gedrag (Payne, 2005).

In Hoofdstuk 3 werd daarom onderzocht in welke mate impliciete alcoholgerelateerde cognities adolescent binge drinken (vijf of meer standaard alcoholconsumpties per gelegenheid) na één jaar konden voorspellen naast andere variabelen, zoals expliciete alcoholgerelateerde cognities. De hiërarchische regressie analyses lieten zien, dat over het algemeen indirecte maten significant iets toevoegde aan de voorspelling van binge drinken na één jaar, terwijl de directe maten (als een groep) dit niet deden. Echter, in het getrimde regressie model is te zien dat expliciete negatieve alcohol verwachtingen, naast klas en impliciete arousal associaties met alcohol, binge drinken voorspelden na één jaar. Bovendien, werd er een drie-weg interactie tussen klas, geslacht en impliciete negatieve alcohol associaties gevonden die significant binge drinken na één jaar voorspelde. Daarnaast, was er ook een trend, dat de tweeweg interactie tussen geslacht en impliciete positieve alcohol associaties prospectief 
binge drinken voorspelde. Kortom, zowel impliciete als expliciete alcoholgerelateerde cognitie voorspelden een uniek deel van de variantie in adolescent binge drinken.

Daaruit volgt dat de rol van expliciete alcohol verwachtingen in het voorspellen van binge drinken na één jaar misschien anders en kleiner is dan eerder werd verondersteld. Allereerst liet het hiërarchische regressie model zien dat in het algemeen de directe maten als een groep niet significant bijdroegen tot de voorspelling van binge drinken na één jaar. Daarnaast lijkt de rol van expliciete alcohol verwachtingen anders dan voorheen werd verondersteld, aangezien in het getrimde regressie model niet positieve of arousal alcohol verwachtingen (Dunn \& Goldman, 1996; 1998; 2000), maar negatieve alcohol verwachtingen significant binge drinken voorspelde na één jaar. Dit komt overeen met het idee dat negatieve verwachtingen een essentiële rol spelen in een preventieve aanpak van huidig en toekomstig alcoholgebruik (Jones, Corbin \& Fromme, 2001). Bovendien, laten de resultaten zien dat impliciete alcohol associaties een belangrijke rol spelen in de vroege ontwikkeling van drinkgedrag. Het hiërarchische regressie model liet zien dat de indirecte maten significant bijdroegen aan de voorspelling van binge drinken na één jaar, waarbij er gecontroleerd wed voor geslacht, klas en expliciete alcohol verwachtingen. Dit is komt overeen met eerder onderzoek naar de invloed van impliciete en expliciete alcoholgerelateerde cognitie in oudere adolescenten (Stacy, Ames, Sussman \& Dent, 1996).

Impliciete alcoholgerelateerde cognitie kun je met verschillende indirecte maten uit diverse onderzoeksparadigma's meten. Daarom stond in Hoofdstuk 4 de vraag centraal, hoe drie van deze indirecte maten (de Impliciete Associatie Test, de Extrinsieke Affectieve Simon Taak en de woord associatie taken) zouden presteren in de voorspelling van alcoholgebruik bij risicojongeren. De resultaten laten zien dat de indirecte maten een uniek deel van de variantie verklaarde in de voorspelling van prospectief alcoholgebruik waarbij gecontroleerd werd voor de directe maat van alcoholgerelateerde cognitie en achtergrondvariabelen. Dit komt overeen met eerder onderzoek naar impliciete en expliciete alcoholgerelateerde cognitieve processen in jong volwassenen en adolescenten (Palfai \& Wood, 2001; Stacy, 1997; Thush \& Wiers, 2007; Wiers, Van Woerden, Smulders \& De Jong, 2002). Daarnaast liet het getrimde model zien, dat woord associatie taken de sterkste voorspellers van alcoholgebruik waren binnen de groep van indirecte maten. Soortgelijke bevindingen werden gerapporteerd in de vergelijking van woord associatie taken, de IAT en de EAST in de voorspelling van marihuana gebruik onder risicojongeren in de U.S. (Ames et al., 2007). Hetzelfde patroon van resultaten werd dus gevonden over verschillende landen, talen en middelen heen.

Een mogelijke verklaring voor dit patroon van resultaten is dat bijvoorbeeld de IAT relatieve associaties meet tussen vooraf bepaalde categorieën. Daarentegen is er in de woord associatie taken vrije competitie mogelijk tussen geassocieerde concepten als antwoord op verscheidene cues. Doordat deze woord associatie taken geen beperking qua voorafbepaalde categorieën opleggen aan het individu, wordt het 
waarschijnlijker dat deze taken daadwerkelijk individuele verschillen in onderliggende motivationele associatieve structuren meten. Aan de ene kant lijken reactie tijd taken beperkt, aangezien er een beperking wordt opgelegd aan de activatie van associatieve structuren. Maar aan de andere kant, kunnen deze vooraf bepaalde categorieën ook nuttig zijn, wanneer specifieke hypotheses over de inhoud van deze alcoholgerelateerde associaties worden getoetst. Kortom, beide soorten indirecte maten (woord associatie taken en reactie tijd taken) hebben hun eigen unieke eigenschappen en voordelen, waarvoor ze gebruikt kunnen worden in verslavingsonderzoek.

De relatie tussen alcoholgerelateerde cognitie en adolescent alcoholgebruik werd geacht te worden gemodereerd door andere factoren, zoals executief functioneren en motivatie. Hoofdstuk 5 beschrijft daarom een studie waarin zowel de interactie tussen impliciete alcoholgerelateerde cognitie en werkgeheugencapaciteit en expliciete alcoholgerelateerde cognitie en werkgeheugen capaciteit werden onderzocht in de voorspelling van alcoholgebruik in risicojongeren. De resultaten van de hiërarchische regressie analyse liet zien dat beide interacties een uniek deel van de variantie verklaarden in de follow-up na één maand waarbij gecontroleerd werd voor de hoofdeffecten en achtergrondvariabelen. Opmerkelijk was dat beide interacties in tegengestelde richting gebruik voorspelden. Impliciete positieve-arousal cognities voorspelde namelijk alcoholgebruik na één maand sterker in proefpersonen met een beneden gemiddelde werkgeheugen capaciteit, terwijl expliciete positieve-arousal cognities gebruik na één maand sterker voorspelden is proefpersonen met een bovengemiddelde werkgeheugen capaciteit.

Deze resultaten komen overeen met eerder onderzoek naar het modererende effect van executieve functies op impliciete cognitieve processen. Het is aangetoond dat impliciete cognitie gedrag vooral voorspelt in personen met relatieve lage werkgeheugen capaciteit of attentionele controle (Grenard et al, in press; Payne, 2005). Daarnaast komen onze bevindingen overeen met de eerdere bevinding van Tapert, McCarthy, Aarons, Schweinsburg en Brown (2003), dat expliciete positieve alcohol verwachtingen vooral alcoholgebruik in probleemdrinkende adolescenten met goede verbale vaardigheden voorspelden, terwijl dit bij probleemdrinkende adolescenten met slechte verbale vaardigheden niet het geval was. Verbale vaardigheden zijn nodig voor het ontwikkelen van het vermogen om in zichzelf te kunnen redeneren (Luria, 1961). Beide resultaten geven aan dat alcohol verwachtingen een diepe verwerking nodig hebben om het maken van beslissingen en het drinkgedrag wat daaruit volgt te beïnvloeden. Tot slot, zijn onze resultaten in overeenstemming met onderzoek dat de dissociatie tussen impliciete en expliciete cognities heeft laten zien. Eerder onderzoek heeft bijvoorbeeld aangetoond, dat expliciete cognitie meer doelmatig gedrag voorspelde, terwijl impliciete cognitie meer spontaan gedrag voorspelde (Asendorpf, Banse \& Mücke, 2002; Perugini, 2005). Onze resultaten zijn eveneens in overeenstemming met het idee dat impliciete en expliciete cognitieve processen gedrag beïnvloeden via verschillende wegen (Fazio \& Towles-Schwen, 1999; Kahne- 
man, 2003; Strack \& Deutsch, 2004) wat overduidelijk relevant is voor verslavingsgedrag (zie Wiers \& Stacy, 2006). Kortom, zowel de interactie tussen werkgeheugencapaciteit en impliciete positieve-arousal cognities en de interactie tussen werkgeheugencapaciteit en expliciete positieve-arousal cognities zouden in ogenschouw moeten worden genomen bij de ontwikkeling van preventie- en interventiemethodes.

Dit impliceert namelijk dat verschillende interventiemethodes effectief kunnen zijn voor verschillende subgroepen van risicojongeren. Aan de ene kant, lijken risicojongeren met een relatief goede werkgeheugencapaciteit gebaat bij interventies die de beschermende negatieve alcohol verwachtingen versterken. Bijvoorbeeld door een motivationeel interview, dat een veelbelovende methode bij risicojongeren lijkt te zijn (Grenard et al, 2007). Aan de andere kant, zouden risicojongeren met een relatief slechte werkgeheugencapaciteit gebaat zijn bij interventies die direct inspelen op hun automatische associaties of reacties op alcohol (bijvoorbeeld attentionele trainingen; zie Wiers et al., 2006) of interventies die tot doel hebben de impulsregulatie te versterken, zoals een werkgeheugen training, die succesvol is bij kinderen met ADHD (Klingberg et al, 2005). Echter, het moet worden gezegd, dat zelfs als de werkgeheugencapaciteit van deze risicojongeren kan worden verbeterd, dit nog niet het belang van motivatie en doelen vitvlakt: de adolescent zal nog steeds gemotiveerd moeten zijn of een duidelijk doel voor ogen moeten hebben om deze executieve controle te kunnen vitoefenen (Feldman-Barrett, Tugade \& Engle, 2004 ; Wiers et al, 2007). Toekomstig onderzoek zal moeten vitwijzen welke methodes als effectieve elementen kunnen worden opgenomen in interventie programma's.

In Hoofdstuk 6 hebben we vervolgens onderzocht wat de invloed Motivationeel Interviewen (MI) was op impliciete en expliciete alcoholgerelateerde cognitie en alcoholgebruik in risicojongeren. Geredeneerd vanuit duale proces modellen van verslaving (Stacy et al., 2004; Wiers et al, 2007), zou ingrijpen op het niveau van motivatie en/of doelbewuste expliciete redenen om te drinken zou dus moeten leiden tot een verandering van drinkgedrag. Desondanks, vonden we geen bewijs dat de huidige MI interventie motivatie om te veranderen of drinkgedrag beïnvloedde, hoewel MI over het algemeen wordt verondersteld de bereidheid om te veranderen te vergroten en dit weer sterk verband houd met het veranderen van gedrag (Miller \& Rollnick, 2002). Onze bevindingen werpen een aantal vragen op over de bruikbaarheid van MI binnen deze specifieke populatie van risicojongeren en de mechanismen waardoor MI mogelijkerwijs werkt. Toekomstig experimenteel onderzoek naar de invloed van MI op alcoholgerelateerde cognitie kan mogelijkerwijs antwoorden geven op deze vragen.

Echter, het concept motivatie vervangt het belang van executieve controle niet (Towles-Schwen \& Fazio, 2006), net zo goed als het concept van executief functioneren niet het belang vervangt van het hebben van voldoende motivatie en doelen (Feldman-Barrett et al., 2004; Wiers et al, 2007). Tevens is er bewijs dat deze twee moderatoren elkaar ook nog eens beïnvloeden. Fadardi en Cox (in Wiers et al., 2006) hebben laten zien dat motivatie om te veranderen kan worden versterkt door de zelfcontrole te 
vergroten. Dit laat zien dat deze twee factoren, die verondersteld worden een modererende werking te hebben op impliciete processen, niet afzonderlijk maar juist gezamenlijke onderzocht dienen te worden in verslavingsonderzoek.

Concluderend kunnen we zeggen, dat de adolescentie een periode is waarin er een verhoogde kwetsbaarheid is om zich met risicovolle activiteiten, zoals overmatig alcoholgebruik, in te laten. Onderzoek heeft nieuwe inzichten gebracht met betrekking tot de cognitieve processen, die dit adolescent drinkgedrag mogelijkerwijs voeden. Zowel impliciete appetitieve associatieve alcoholgerelateerde processen als expliciete reflectieve alcoholgerelateerde processen beïnvloeden adolescent drinkgedrag. De directe positieve-arousal effecten van alcohol lijken als relatief automatische associaties drinkgedrag te sturen, terwijl meer indirecte negatieve effecten van drinken meer tijd, moeite en reflectie lijken nodig te hebben om drinkgedrag te kunnen beïnvloeden. Executief functioneren modereert deze relatie tussen alcoholgerelateerde cognitie en adolescent alcoholgebruik. Er is meer onderzoek nodig om vast te stellen of inderdaad negatieve reflectieve processen de invloed van impliciete appetitieve associatieve processen modereren, wanneer de persoon gemotiveerd genoeg is en voldoende executieve capaciteit heeft. Sommige van de opgeworpen vragen in dit proefschrift laten zien, dat de veronderstellingen van duale proces modellen van verslaving en de implicaties voor de ontwikkeling van nieuwe interventies nog in meer detail moeten worden onderzocht. 
SAMENVATTING 



\section{References}

Allen, J. P, Porter, M. R., McFarland, F. C., Marsh, P, \& McElhaney, K. (2005). The two faces of adolescents success with peers: Adolescent popularity, social adaptation and deviant behavior. Child Development, 76, $747-760$.

Ames, S. L., Grenard, J. L., Thush, C., Sussman, S., Wiers, R. W. \& Stacy, A. W. (2007). Comparison of indirect assessments of marijuana-related associations among at-risk adolescents. Experimental Clinical Psychopharmacology, 15, 204-218.

Ames, S. L., Sussman, S., Dent, C. W., \& Stacy, A. W. (2005). Implicit cognition and dissocative experiences as predictors of adolescent substance use. American Journal of Drug \& Alcohol Abuse, 31, 129-162.

Ames, S. L., Zogg, J. B., \& Stacy, A. W. (2002). Implicit cognition, sensation seeking, marijuana use and driving behavior among drug offenders. Personality \& Individual Differences, 33, 1055-1072.

Anderson, K. G., Schweinsburg, A., Paulus, M. P., Brown, S. A., \& Tapert, S. (2005). Examining personality and alcohol expectancies using functional magnetic resonance imaging (fMRI) with adolescents. Journal of Studies on Alcohol, 66, $323-331$.

Arbuckle, J. L., \& Wothke, W. (1999). AMOS 4.0 user's guide. Chicago: SPSS Press.

Arbuckle, J. L. (2006) AMOS 7.0 user's guide. Chicago: SPSS Press.

Arnett, J. J. (1999). Adolescent storm and stress, reconsidered. American Psychologist, 54, 317 -326.

Asendorpf, J. B., Banse, R., \& Mücke, D. (2002). Double dissociation between implicit and explicit personality self-concept: The case of shy behavior. Journal of Personality and Social Psychology, 83, 380-393.

Azjen, I. (1991). The theory of planned behavior. Organizational Behavior and Human Decision Processes, 50, 179-221.

Bear, J.S., Marlatt, G. A., Kivlahan, D. R., Fromme, K., Larimer, M. E., \& Williams, E. (1992). An experimental test of three methods of alcohol risk reduction with young adults. Journal of Consulting and Clinical Psychology, 60, $974-979$.

Bechara, A. (2005). Decision making, impulse control and loss of willpower to resist drugs: A neurocognitive perspective. Nature Neuroscience, 8, 1459-1463.

Beringer, J. (1996). Experimental Run Time System (ERTS), Version 3. 18. Frankfurt, Germany: Berisoft.

Bosari, B. \& Carey, K. B. (2000). Effects of a brief motivational intervention with college student drinkers. Journal of Consulting and Clinical Psychology, 68, 728-733.

Bosson, J. K., Swann, W. B., \& Pennebaker, J. (2000). Stalking the perfect measures of implicit selfesteem: The blind man and the elephant revisited? Journal of Personality and Social Psychology, 79, 631-643.

Botvin, G. J., Griffin, K. W. (2004). Life skills training: Empirical predictions and future directions. Journal of Primary Prevention, 25, 211-232.

Botvin, G. J., Griffin, K. W., Diaz, T., \& Ifill-Williams, M. . (2001) Preventing binge drinking during early adolescence: One- and two-year follow-up of a school based preventive intervention. Psychology of Addictive Behavior, 15, 360-365.

Brown, S. A., Tapert, S. F., Granholm, E., \& Dellis, D. C. (2000). Neurocognitive functioning of adolescents: Effects of protracted alcohol use. Alcohol: Clinical and Experimental Research, 24, 164171. 


\section{REFERENCES}

Cameron, C. A., Stritzke, W. G. K. \& Durkin, K. (2003). Alcohol expectancies in late childhood: An ambivalence perspective on transitions toward alcohol use. Journal of Child Psychology and Psychiatry, 44, 687-698.

Chassin, L., Presson, C. C., Rose, J. S., Sherman, S. J., \& Prost, J. (2002) Parental smoking cessation and adolescent smoking. Journal of Pediatric Psychology, 27, $485-496$.

Chinn, C. A., \& Brewer, W. F. (1993). The role of anomalous data in knowledge acquisition: A theoretical framework and implications for science instruction. Review of Educational Research, 63, $1-49$.

Cohen, J., \& Cohen, P. (1983). Applied multiple regression/correlation analysis for the behavioral sciences (2nd ed.). Hillsdale: L. Erlbaum Associates.

Conrod, P. J., Stewart, S. H., \& Pihl, R. O. (2000) Efficacy of brief coping skills interventions that match different personality profiles of female substance abusers. Psychology of Addictive Behavior, 14, 231-242.

Cox, W. M., \& Klinger, E. (2002). Motivational structure relationships with substance use and processes of change. Addictive Behaviors, 27, 925 - 940.

Crews, F. T., Braun, C. J, Hoplight, B., Switzer, R.C. 3rd, \& Knapp D. J. (2000). Binge ethanol consumption causes differential brain damage in young adolescent rats compared with adult rats. Alcoholism: Clinical and Experimental Research, 24, 1712-1723.

Cuijpers, P., Jonkers, R., De Weerdt, I., \& De Jong, A. (2002). The effects of drug abuse prevention at school: The 'Healthy School and Drugs' project. Addiction, 97, 67-73.

Dahl, R. E. (2004). Adolescent brain development: A period of vulnerabilities and opportunities. Annals of the New York Academy of Sciences, 1021, 1-22.

Darkes, J., \& Goldman, M. S. (1993). Expectancy challenge and drinking reduction: Experimental evidence for a mediational process. Journal of Consulting Clinical Psychology, 61, 344-353.

Darkes, J., \& Goldman, M. S. (1998). Expectancy challenge and drinking reduction: Process and structure in the alcohol expectancy network. Experimental Clinical Psychopharmacology, 6, 6476.

De Bellis, M.D., Clark, D.B., Beers, S.R., Soloff, P.H., Boring A.M., Hall, J. et al. (2000). Hippocampal volume in adolescent-onset alcohol use disorders. American Journal of Psychiatry, 157, 737-744.

Deckel, A. W., Hesselbrock, V., \& Baver, L. (1995). Relationship between alcohol-related expectancies and anterior brain functioning in young men at risk for developing alcoholism. Alcoholism: Clinical and Experimental Research, 19, 476-481.

De Houwer, J. (2001). A structural and process analysis of the Implicit Association Test. Journal of Personality and Social Psychology, 37, 443-451.

De Houwer, J. (2002). The Implicit Association Test as a tool for studying dysfunctional associations in psychopathology: Strengths and limitations. Journal of Behavior Therapy and Experimental Psychiatry, 33, 115-133.

De Houwer, J. (2003). The Extrinsic Affective Simon Task. Experimental Psychology, 50, 77 - 85.

De Houwer, J. (2006). What are implicit measures and why are we using them? In R. W. Wiers \& A. W. Stacy (Eds.), Handbook of implicit cognition and addiction. (pp. 11 - 28). Thousand Oaks: SAGE.

De Houwer, J., \& De Bruycker, E. (2007). The Implicit Association Test outperforms the Extrinsic Affective Simon Task as an implicit measure of interindividual differences in attitudes. British Journal of Social Psychology, 46, $401-421$.

De Houwer, J., Baeyens, F., \& Field, A. P. (2005). Associative learning of likes and dislikes: Some current controversies and possible ways forward. Cognition and Emotion, 19, 161- 174. 
De Houwer, J., Crombez, G., Koster, E. H. W., \& De Beul, N. (2004). Implicit alcohol-related cognitions in clinical samples of heavy drinkers. Journal of Behaviour Therapy and Experimental Psychiatry, 35, 275-286.

De Jong, P. J., Wiers, R.W., van den Braak, M., Huijding, J. (2007). Using the Extrinsic Affective Simon Test as a measure of implicit attitudes towards alcohol: Relationship with drinking behavior and alcohol problems. Addictive Behaviors, 32, 881-887.

Deutsch, R., Gawronski, B., \& Strack, F. (2006) At the boundaries of automaticity: Negation as reflective operation. Journal of Personality and Social Psychology, 91, 385-405.

Deutsch, R. \& Strack, F. (2006). Reflective and impulsive determinants of addictive behavior. In R. W. Wiers \& A. W. Stacy (Eds.), Handbook of implicit cognition and addiction. (pp. 45 - 58). Thousand Oaks: SAGE.

Di Chiara, G. (2000). Role of dopamine in the behavioral actions of nicotine related to addiction. European Journal of Pharmacology, 393, 295-314.

Dimeff, L. A., Bear, J. S., Kivlahan, D. R., \& Marlatt, G. A. (1999). BASICS: Brief Alcohol Screening and Intervention for College Students: A harm reduction approach. New York: Guilford Press.

Dunn, M. E., \& Goldman, M. S. (1996). Empirical modeling of an alcohol expectancy memory network in elementary school children as a function of grade. Experimental Clinical Psychopharmacology, 4, $209-217$.

Dunn, M. E., \& Goldman, M. S. (1998). Age and drinking-related differences in the memory organization of alcohol expectancies in $3^{\text {rd }}-6^{\text {th }}-9^{\text {th }}$, and $12^{\text {th }}$ - grade children. Journal of Consulting and Clinical Psychology, 66, 579-585.

Dunn, M. E., \& Goldman, M. S. (2000). Validation of multidimensional scaling-based modeling of alcohol expectancies in memory: Age and drinking-related differences in expectancies of children assessed as first associates. Alcoholism: Clinical and Experimental Research, 24, 1639 1646.

Erickson, K.I, Colcombe, S.J., Wadhwa, R., Bhere, L., Peterson, M.S., Scalf, P.E., Kim, J.S., Alvarado, M., \& Kramer, A.F. (2007). Training-induced functional activation in dual task processing: An fMRI study. Cerebral Cortex. 17, $192-204$.

Fazio, R.H., \& Towles-Schwen, T. (1999). The MODE model of attitude-behavior processes. In S. Chaiken \& Y. Trope (Eds.), Dual process theories in social psychology (pp. 97-116). New York: Guilford Press.

Feldman-Barrett, L.F., Tugade, M.M., \& Engle, R.W. (2004). Individual differences in working memory capacity and dual-process theories of the mind. Psychological Bulletin, 130, 553-573.

Finn, P.R., \& Hall, J. (2004). Cognitive ability and risk for alcoholism: Short-term memory capacity and intelligence moderate personality risk for alcohol problems. Journal of Abnormal Psychology, $113,569-581$.

Foxcroft, D. R., Ireland, D., Lister-Sharp, D. J, Lowe, G., \& Breen, R. (2003). Longer-term primary prevention for alcohol misuse in young people: a systematic review. Addiction, 98, $397-411$.

Fromme, K., Marlatt, G. A., Baer, J. S., \& Kivlahan, D. R. (1994). The alcohol skills training program: Group intervention for young adult drinkers. Journal of Substance Abuse Treatment, 11, 143-154.

Gardner, M. \& Steinberg, L. (2005). Peer influence on risk taking, risk preference and risky decision making in adolescence and adulthood: An experimental study. Developmental Psychology, 41 , $625-635$.

Gawronski, B., \& Bodenhausen, G. V. (2006) Associative and propositional processes in evaluation: An integrative review of implicit and explicit attitude change. Psychological Bulletin, 132, 692731. 


\section{REFERENCES}

Goldman, M. S. \& Darkes, J. (2004). Alcohol expectancy multiaxial assessment: A memory networkbased approach. Psychological Assessment, 16, 4-15.

Goldman, M. S., Del Boca, F. K. \& Darkes, J. (1999). Alcohol expectancy theory: The application of cognitive neuroscience. In H. T. Blane, \& K. E. Leonard (Eds.), Psychological theories of drinking and alcoholism (2nd ed., pp. 203-246). New York: Guilford Press.

Grant, B. F., \& Dawson, D. A. (1997). Age at onset of alcohol use and its association with DSM-IV alcohol abuse and dependence: results from the national longitudinal alcohol epidemiologic survey. Journal of Substance Abuse, 9, 103-110.

Greenwald, A. G., \& Banaji, M. R. (1995). Implicit social cognition: Attitudes, self-esteem, and stereotypes. Psychological Review, 102, 4-27.

Greenwald, A. G., McGhee, D. E., \& Schwartz, J. L. K. (1998). Measuring individual differences in implicit cognition: The Implicit Association Test. Journal of Personality and Social Psychology, 74, 1464-1480.

Greenwald, A. G., Nosek, B. A., \& Banaji, M. R. (2003). Understanding and using the Implicit Association Test: 1. An improved scoring algorithm. Journal of Personality and Social Psychology, 85, 197-216.

Grenard, J. L., Ames, S. L., Pentz, M. A., \& Sussman, S. (2006). Motivational interviewing with adolescents and young adults for drug-related problems. International Journal of Adolescent Medical Health, 18, 53 -67.

Grenard, J. L., Ames, S. L., Wiers, R. W., Thush, C., Stacy, A.W., \& Sussman, S. (2007). Brief intervention for substance use among at-risk adolescents: An initial study. Journal of Adolescent Health, 40, 188-191.

Grenard, J.L., Ames, S.L, Wiers, R.W., Thush, C., Sussman, S., \& Stacy, A.W. (in press). Working memory moderates the predictive effects of drug-related associations on substance use. Psychology of Addictive Behavior.

Hess, B. (2000) Assessing program impact using latent growth modeling: A primer for the evaluator. Evaluation and Program Planning, 23, 419-428.

Hibell, B., Andersson, B., Bjarnason, T., Ahlström, S., Balakireva, O., Kokkevi, A., et al. (2004). The ESPAD report 2003: Alcohol and other drug use among students in 35 European countries. Stockholm: The Swedish Council for Information on Alcohol and Other Drugs (CAN) and the Pompidou Group at the Council of Europe.

Hofmann, W., Rauch, W., \& Gawronski, B. (2007). And deplete us not into temptation: Automatic attitudes, dietary restraint, and self-regulatory resources as determinants of eating behavior. Journal of Experimental Social Psychology, 43, 497-504.

Houben, K. (2007). Decoding the alcohol-IAT: The Implicit Association Test as a measure of individual differences in implicit preferences for alcohol. Unpublished doctoral dissertation.

Houben, K., \& Wiers, R. W. (2006). Assessing implicit alcohol associations with the Implicit Association Test: Fact or artifact? Addictive Behaviors, 31, 1346- 1362.

Huijding, J. \& De Jong, P. (2006). Automatic associations with the sensory aspects of smoking: Positive in habitual smokers but negative in non-smokers. Addictive Behaviors, 31, 182-186.

Huijding, J., \& De Jong, P. (2007). Beyond fear and disgust: The role of (automatic) contaminationrelated associations in spider phobia. Journal of Behavior Therapy and Experimental Psychiatry, $38,200-211$.

Jajodia, A., \& Earleywine, M. (2003). Measuring alcohol expectancies with the Implicit Association Test. Psychology of Addictive Behaviors, 17, 126-133.

Jones, B. T., Corbin, W. \& Fromme, K. (2001). A review of expectancy theory and alcohol consumption. Addiction, 91, 57-72. 
Jones, B. C., Jones, B. T., Blundell, L. \& Bruce, G. (2002). Social users of alcohol and cannabis who detect substance-related changes in a change blindness paradigm report higher levels of use than those detecting substance-neutral changes. Psychopharmacology, 165, $93-96$.

Jones, B. T., Jones, B. C., Smith, H. \& Coply, N. (2003). A flicker paradigm for inducing change blindness reveals alcohol and cannabis information processing biases in social users. Addiction, 98, 235-244.

Jones, B. T., \& McMahon, J. (1996). A comparison of positive and negative alcohol expectancy and value and their multiplicative composite as predictors of post- treatment abstinence survivorship. Addiction, 91, 89 - 99.

Jones, B. T., \& McMahon, J. (1998). Alcohol motivations as outcome expectancies. In W. R. Miller \& N. Heather (Eds.), Treating addictive behaviors. Applied clinical psychology (2nd ed.) (pp. 75-91). New York: Plenum Press.

Kahneman, D. (2003). A perspective on judgment and choice. American Psychologist, 58, 697-720.

Kalivas, P.W., \& Volkow, N.D. (2005). The neural basis of addiction: A pathology of motivation and choice. American Journal of Psychiatry, 162, 1403-1413.

Kane, M.J., \& Engle, R.W. (2002). The role of prefrontal cortex in working-memory capacity, executive attention, and general fluid intelligence: An individual-differences perspective. Psychonomic Bulletin \& Review, 9, 637-671.

Karpinski, A. \& Steinman, R. B (2006). The single category implicit association test as a measure of implicit social cognition, Journal of Personality and Social Psychology, 91, 16-32

Kelly, A. B., Masterman, P. W., \& Marlatt, G. A. (2005). Alcohol-related associative strength and drinking behaviours: Concurrent and prospective relationships. Drug and Alcohol Review, 24, 489-498.

Klingberg, T., Fernell, E., Olesen, P. J., Johnsons, M., Gustafsson, P., Dahlström, K. Gillberg, C. G., Forssberg, H., \& Westerberg, L. P. (2005). Computerized training of working memory in children with ADHD: A randomized, controlled trial. Journal of the American Academy of Child and Adolescent Psychiatry, 44, 177 - 186.

LaBrie, J. W., Quinlan, T., Schiffman, J. E., \& Earleywine, M. E. (2005) Performance of alcohol and safer sex change rulers compared with readiness to change questionnaires. Psychology of Addictive Behaviors, 19, 112-115

Lang, P. J. (1995). The emotion probe. Studies of motivation and attention. American Psychologist, $50,372-385$.

Larimer, M. E., \& Cronce, J. M. (2002). Identification, prevention and treatment: A review of individual-focused strategies to reduce problematic alcohol consumption by college students. Journal of Studies on Alcohol, 14 (Suppl 1), 148-163.

Larimer, M.E., \& Cronce, J. M. (2007). Identification, prevention and treatment revisited: Individualfocused college drinking prevention strategies 1999-2006. Addictive Behaviors, 32, 2439-2468.

Leigh, B. C. (1989). In search of the seven dwarves: Issues of measurement and meaning in alcohol expectancy research. Psychological Bulletin, 105, 361-373.

Luna, B., Thulborn, K. R., Munoz, D. P., Merriam, E. P., Garver, K.E., Minshew, N.J., et al. (2001). Maturation of widely distributed brain function subserves cognitive development. Neuroimage, 13, 786 $-793$.

Luria, A.R. (1961). The role of speech in the regulation of normal and abnormal behavior. New York: Liveright Publications.

Marlatt, G.A., Baer, J.S., Kivlahan, D.R., Dimeff, L.A., Larimer, M.E., Quigley, L.A. et al. (1998). Screening and brief intervention for high-risk college student drinkers: Results from a 2-year follow-up assessment. Journal of Consulting and Clinical Psychology, 66, 604-615. 


\section{REFERENCES}

McClelland, G. H., \& Judd, C. M. (1993). Statistical difficulties of detecting interactions and moderator effects. Psychological Bulletin, 114, 376-390.

Meredith, W., \& Tisak J. (1990). Latent curve analysis. Psychometrika, 55, 107-122.

Mierke, J. \& Klaver, C. J. (2003). Method-specific variance in the implicit association test. Journal of Personality and Social Psychology, 85, 1180-1192.

Miller, W. R., Brown, J. M., Simpson, T. L., Handmaker, N. S., Bien, T. H., Luckier, L. F. et al. (1995). What works? A methodological analysis of alcohol treatment outcome literature. In R. K. Hester \& W. R Miller (Eds.) Handbook of alcoholism treatment approaches. (pp. 12-44). Boston: Allyn and Bacon.

Miller, W.R. \& Rollnick, S. (2002). Motivational Interviewing: Preparing people to change addictive behaviors (2nd ed.). New York: Guilford Press.

Miyake, A., Friedman, N.P., Emerson, M.J., Witzki, A.H., Howerter, A., \& Wager, T.D. (2000). The unity and diversity of executive functions and their contributions to complex "frontal lobe" tasks: A latent variable analysis. Cognitive Psychology, 41, 49-100.

Monshouwer, K., Van Dorsselaer, S., Gorter, A., Verdurmen, J., \& Vollebergh, W. (2004). Jeugd en riskant gedrag. Kerngegevens uit het peilstationsonderzoek 2003. Roken, drinken, drugsgebruik en gokken onder scholieren vanaf tien jaar. Utrecht: Trimbos-institute.

Moyer, A., Finney, J., Swearingen, C, \& Vergun, P. (2002). Brief interventions for alcohol problems: A meta-analytic review of controlled investigations in treatment-seeking and non-treatmentseeking populations. Addiction, 97, 279-92.

Moyers, T. B., Martin, T., Manuel, J. K., Hendrickson, S. M. L. \& Miller, W. R. (2005). Assessing competence in the use of motivational interviewing. Journal of Substance Abuse Treatment, 28, 19- 26.

National Institute on Alcohol Abuse and Alcoholism (NIAAA) (2005). The effects of alcohol on physiological processes and biological development. Alcohol Research and Health, 28, 125-131.

Newcomb, M.D., \& Bentler, P.M. (1989). Substance use and abuse among children and teenagers. American Psychologist, 44, 242-248.

Nisbett, R. E., \& Wilson, T. D. (1977). Telling more than we can know: Verbal reports on mental processes. Psychological Review, 84, 231-259.

O'Conner, R. M ., Fite, P. J. , Nowlin, P. R., \& Colder, C. R. (2007). Children's belief about substance use: An examination of age differences in implicit and explicit cognitive precursors of substance use initiations. Psychology of Addictive Behaviors, 21, 525- 533.

Olesen, P. J., Westerberg, H. \& Klingberg, T. (2004). Increased prefrontal and parietal activity after training of working memory. Nature Neuroscience, 7, $75-79$.

Olson, M.A., \& Fazio, R. H. (2004). Trait interferences as a function of automatically activated racial attitudes and motivation to control prejudiced reactions. Basic Applied Social Psychology, 26, 1 $-11$

Palfai, T., \& Wood, M. D. (2001). Positive alcohol expectancies and drinking behavior: The influence of expectancy strength and memory accessibility. Psychology of Addictive Behaviors, 15, 60-67.

Payne, B.K. (2005). Conceptualizing control in social cognition: How executive functioning modulates the expression of automatic stereotyping. Journal of Personality and Social Psychology, 89, 488-503.

Perugini, M. (2005). Predictive models of implicit and explicit attitudes. British Journal of Social Psychology, 44, 29-45.

Peterson, J.B., Finn, P.R., \& Pihl, R.O. (1992). Cognitive dysfunction and the inherited predisposition to alcoholism. Journal of Studies on Alcohol, 53, 154-160.

Peterson, J.B., Pihl, R.O., Higgins, D., \& Lee, A. (2002). NeuroCognitive Battery. Version 2.0. Deerfield Beach. 
Petrides, M., \& Milner, B. (1982). Deficits on subject-ordered tasks after frontal- and temporal-lobe lesions in man. Neuropsychologia. 20, 249-262.

Poelen, E. A. P., Scholte, R. H. J., Engels, R. C. M. E, Boomsma, D. I, \& Willemsen G. (2005). Prevalence and trends of alcohol use and misuse among adolescents and young adults in the Netherlands from 1993 to 2000. Drug and Alcohol Dependence, 79, 413-421.

Project Match Research Group (1997). Project Match secondary a priori hypotheses. Addiction 92 , 1671-1698.

Rensink, R.A. (2000). Change detection. Annual Review of Psychology, 53, 245-277.

Rinck, M., \& Becker, E. S. (2007) Approach and avoidance in fear of spiders. Journal of Behavioral Therapy and Experimental Psychiatry, 38, 105-120.

Robinson, T. E., \& Berridge, K. C. (1993). The neural basis of drug craving: An incentive-sensitization theory of addiction. Brain Research Reviews, 18, 247-291.

Rothermund, K. \& Wentura, D. (2004). Underlying processes in the Implicit Association Test (IAT): Dissociating salience from associations. Journal of Experimental Psychology: General, 133, 139165.

Rudman, L. A. (2004). Sources of implicit attitudes. Current Directions in Psychological Science, 13, 79-82.

Schuckit, M. A. (1998). Biological, psychological and environmental predictors of the alcoholism risk: A longitudinal study. Journal of Studies on Alcohol, 59, 485-494.

Schwartz, N. (2000). Emotion, cognition, and decision making. Cognition and Emotion, 14, 433-440.

Shalev, L., Tsal, Y., Mevorach, C. (2007). Computerized progressive attentional training (CPAT) program; Affective direct intervention for children with ADHD. Child Neuropsychology, 13, 382 388.

Sher, K. J., \& Gotham, H. J. (1999). Pathological alcohol involvement: A developmental disorder of young adulthood. Development and Psychopathology, 11, 933-956.

Sher, K. J., Wood, M. D., Wood, P. K., \& Raskin, G. (1996). Alcohol outcome expectancies and alcohol use: A latent variable cross-lagged panel study. Journal of Abnormal Psychology, 105, 561-574.

Sobell, L. C., \& Sobell, M. B. (1990). Self-report issues in alcohol-abuse: State of the art and future directions. Behavioral Assessment, 12, 77-90.

Spear, L. P. (2004). Adolescence and the trajectory of alcohol use: Introduction to part VI. Annals of the New York Academy of Sciences, 1021, $202-205$.

Stacy, A. W. (1995). Memory association and ambiguous cues in models of alcohol and marijuana use. Experimental \& Clinical Psychopharmacology, 3, 183-194.

Stacy, A. W. (1997). Memory activation and expectancy as prospective predictors of alcohol and marijuana use. Journal of Abnormal Psychology, 106, 61-73.

Stacy, A. W., \& Newcomb, M. D. (1998). Memory association and personality as predictors of alcohol use: Mediation and moderator effects. Experimental \& Clinical Psychopharmacology, 6, 280291.

Stacy, A. W., Ames, S. L., \& Knowlton, B. J. (2004). Neurologically plausible distinctions in cognition relevant to drug use etiology and prevention. Substance Use and Misuse, 39, 1571-1623.

Stacy, A. W., Ames, S. L., Sussman, S. \& Dent, C. W. (1996). Implicit cognition in adolescent drug use. Psychology of Addictive Behaviors, 10, 190-203.

Stacy, A. W., Galaif, E. R., Sussman, S., \& Dent, C. W. (1996). Self-generated drug outcomes in highrisk adolescents. Psychology of Addictive Behaviors, 10, 18-27.

Stacy, A. W., Newcomb, M. D., \& Bentler, P. M. (1991). Cognitive motivation and drug use: A 9-year longitudinal study. Journal of Abnormal Psychology, 100, 502-515. 


\section{REFERENCES}

Steinberg, L. (2005). Cognitive and affective development in adolescence. Trends in Cognitive Science, 9, $69-74$.

Steinberg, L. (2007). Risk taking in adolescence: New perspectives from brain and behavioral science. Current Directions in Psychological Science, 16, 55 - 58.

Strack, F., \& Deutsch, R. (2004). Reflective and impulsive determinants of social behavior. Personality and Social Psychology Review, 8, $220-247$.

Sussman, S., Stacy, A. W., Ames, S. L., \& Freedman, L. B. (1998). Self-reported high-risk locations of adolescent drug use. Addictive behaviors, 23, 405-411.

Tapert, S. F., Baratta, B. S., Abrantes, B. A., \& Brown, S. A. (2002). Attention dysfunction predicts substance involvement in community youths. Journal of American Academy of Child and Adolescent Psychiatry, 41, 680-686.

Tapert, S. F., McCarthy, D. M., Aarons, G. A., Schweinsburg, A. D., \& Brown, S. A., (2003). Influence of language abilities and alcohol expectancies on the persistence of heavy drinking in youth. Journal of Studies on Alcohol, 64, 313-321.

Tapert, S. F., Schweinsburg, A. D., Barlett, V. C., Brown, S. A., Frank, L. R., Brown, G. G. et al. (2004). Blood oxygen level dependent response and spatial working memory in adolescents with alcohol use disorders. Alcoholism: Clinical and Experimental Research, 28, 1577-1586.

Teachman, B. A., \& Woody, S. R. (2003). Automatic processing in spider phobia: Implicit fear association over the course of treatment. Journal of Abnormal Psychology, 112, 100 -109.

Thorpe, S. J., \& Salkovskis, P. M. (1997). The effect of one-session treatment for spider phobia on attentional bias and beliefs. British Journal of Social and Clinical Psychology, 36, 225-241.

Thush, C. \& Wiers, R.W. (2007). Explicit and implicit alcohol-related cognitions and the prediction of future drinking in adolescents. Addictive Behaviors, 32, 1367-1383.

Thush, C., Wiers, R.W., Ames, S. L., Grenard, J. L., Sussman, S., \& Stacy, A.W. (2007). Apples and oranges? Comparing indirect measures of alcohol-related cognition predicting alcohol use in at-risk adolescents. Psychology of Addictive Behaviors, 21, 587 -591.

Thush, C., Wiers, R. W., Theunissen, N., Van den Bosch, J., Opdenacker, J., van Empelen, P. et al. (2007). A randomized clinical trial of a targeted intervention to moderate alcohol use and alcohol-related problems in at-risk adolescents. Pharmacology Biochemistry and Behavior, 86, 368 376.

Thush, C., Wiers, R.W., Ames, S. L., Grenard, J. L., Sussman, S., \& Stacy, A.W. (2008). Interactions between implicit cognition and working memory in the prediction of.alcohol use in at-risk adolescents. Drug and Alcohol Dependence, 94, 116- 124.

Towles-Schwen, T, \& Fazio, R. H. (2006). Automatically activated racial attitudes as predictors of the success of interracial roommate relationships. Journal of Experimental Social Psychology, 42, 698-705.

Van de Luitgaarden, J., Thush, C., Wiers, R. W., \& Knibbe, R. A. (2008). Prevention of alcohol problems in Dutch youth: Missed opportunities and new developments. Evaluation \& the Health Professions, 31, $167-181$.

Van de Luitgaarden, J., Wiers, R.W., Knibbe, R.A. \& Boon, B.J. (2006). From the laboratory to real life: A pilot study of an expectancy challenge with 'heavy drinking' young people on holiday. Substance Use and Misuse, 41, 353-368.

Van der Vorst, H., Engels, R. C. M. E., Meeus, M., \& Deković, M. (2006). The impact of alcohol-specific rules, parental norms about early drinking and parental alcohol use on adolescents' drinking behavior. Journal of Child Psychology and Psychiatry, 47, 1299-1306.

Van Dijck, D. \& Knibbe, R.A. (2005). De prevalentie van probleemdrinken in Nederland. Maastricht: Universiteit Maastricht. 
Van Kleef, L.E. \& Van der Lely, N. (2006). Jongeren met alcoholintoxicatie: een groeiend probleem. Nederlands Tijdschrift voor Geneeskunde, 150 (46), 2521-2522.

Volkow, N., Fowler, J., \& Wang, G. (2004). The addicted human brain viewed in the light of imaging studies: Brain circuits and treatment strategies. Neuropharmacology, 47, 3-13.

Weinstein, N. D. (1982). Unrealistic optimism about susceptibility to health problems. Journal of Behavioral Medicine, 5, 441-460.

Wellman, H. M., \& Gelman, S. A. (1992). Cognitive development: Foundational theories of core domains. Annual Review of Psychology, 43, 337-375.

White, A. M., Ghia, A. J., Levin, E. D., \& Swartzwelder H. S. (2000). Binge pattern alcohol exposure: Differential impact on subsequent responsiveness to alcohol. Alcoholism: Clinical and Experimental Research, 24, 1251-1256.

White, A.M., \& Swartzwelder, H. S. (2004). Hippocampal function during adolescence: A unique target of ethanol effects. Annals of the New York Academy of Sciences, 102, 206-220.

White, H. R., \& Labouvie, E. W. (1989). Towards the assessment of adolescent problem drinking. Journal of Studies on Alcohol, 50, 30-37.

White, H. R., \& Labouvie, E. W. (2000). Longitudinal trends in problem drinking as measured by the Rutgers Alcohol Problem Index [abstract]. Alcoholism: Clinical and Experimental Research, 24 (5 Suppl 1), 76A.

Wiers, R.W., Bartholow, B.D., van den Wildenberg, E., Thush C., Engels, R., Sher, K.J., Grenard, J.L., Ames, S.L, Stacy, \& A.W. (2007). Automatic and controlled processes and the development of addictive behaviors in adolescents: A review and a model. Pharmacology, Biochemistry and Behavior, 86, $263-283$.

Wiers, R. W., Cox, W. M., Field, M., Fadardi, J. S., Palfai, T. P., Schoenmakers, T. et al. (2006). The search for new ways to change implicit alcohol-related cognitions in heavy drinkers. Alcoholism: Clinical and Experimental Research, 30, 320-331.

Wiers, R.W., De Jong, P. J. Havermans, R., \& Jelicic, M. (2004). How to change implicit drug userelated cognitions in prevention: A transdisciplinary integration of findings from experimental psychopathology, social cognition, memory and experimental learning theory. Substance Use \& Misuse, 39, 1625-1684.

Wiers, R. W., Gunning, W. B., \& Sergeant, J. A. (1998). Do young children of alcoholics hold more positive or negative alcohol-related expectancies than controls? Alcoholism: Clinical and Experimental Research, 22, 1855-1863.

Wiers, R. W., Hoogeveen, K. J., Sergeant, J. A., \& Gunning, W. B. (1997). High and low dose expectancies and the differential associations with drinking in male and female adolescents and young adults. Addiction, 92, 871-888.

Wiers, R. W., Houben, K., Smulders, F. T. Y., Conrod, P. J., \& Jones, B. T. (2006). To drink or not to drink: the role of automatic and controlled cognitive processes in the etiology of alcohol-related problems. In R. W. Wiers \& A. W. Stacy (Eds.), Handbook of implicit cognition and addiction. (pp. 339-361). Thousand Oaks: SAGE.

Wiers, R.W., \& Kummeling, R. H. C (2004). An experimental test of an alcohol expectancy challenge in mixed gender groups of heavy young drinkers. Addictive Behaviors, 29, 215-220.

Wiers, R. W., \& Stacy, A.W. (Eds.), (2006). Handbook of implicit cognition and addiction. Thousand Oaks: SAGE.

Wiers, R. W., Van de Luitgaarden, J., Van den Wildenberg, E., \& Smulders, F. T. Y. (2005). Challenging implicit and explicit alcohol-related cognitions in young heavy drinkers. Addiction, 100, 806 -819.

Wiers, R.W., Van Woerden, N., Smulders, F. T. Y. \& De Jong, P. J. (2002). Implicit and explicit alcoholrelated cognitions in heavy and light drinkers. Journal of Abnormal Psychology, 111, 648-658. 


\section{REFERENCES}

Wigboldus, D. , Holland, R. W. , \& van Knippenberg, A. (2005). Single target implicit associations. Unpublished manuscript.

Wilson, S. J., Sayette, M. A., \& Fiez, J. A., (2004). Prefrontal responses to drug cues: A neurocognitive analysis. Nature Neuroscience, 1, 211-214.

Wood, M., Capone, C., Laforge, R., Erickson, D. J. \& Brand, N. H. (2007) Brief motivational interview and alcohol expectancy challenge with heavy drinking college students: A randomized factorial study. Addictive Behaviors, 32, 2509-2528.

Zuckerman, M., Kuhlman, D. M., Thornquist, M., \& Kiers, H. (1991). Five (or three) robust questionnaire scale factors of personality without culture. Personality \& Individual Differences, 12, 929-941. 



\section{Dankwoord}

Het schrijven van een dankwoord is een mooi voorbeeld van hoe automatische associatieve en meer doelbewuste rationele processen ook goed kunnen samenwerken. Er schieten je natuurlijk als eerste een aantal personen direct in je gedachten. Vaak is het best moeilijk in mooie volzinnen te benoemen wat deze mensen nu precies voor je betekent hebben, maar na lang nadenken wil je er toch graag iets verstandigs over zeggen...

Mijn begeleiders Reinout en Anita wil ik vooral bedanken voor alle kansen die ze me hebben geboden om het maximale vit mijzelf en mijn aio tijd te halen. Ik heb geprobeerd deze kansen met beide handen aan te grijpen en er iets moois mee te doen. Het was bijzonder om in het internationale toponderzoek te mogen meedraaien.

Dank aan mijn directe collega's Katrijn, Tim, Jade en Esther. leder van jullie heeft zo zijn of haar eigen unieke bijdrage geleverd aan een bijzondere tijd. Niet alleen op wetenschappelijk vlak, maar ook bijvoorbeeld op al die mooie congressen vol met disfunctionele föhns, walvissen, onverwachte kamergenoten, zwembadfeestjes en ga zo maar door.

Saskia en Jill, jullie waren niet alleen mij collegaatjes, maar ook veel meer dan dat. Jullie waren bij alle hoogte- en dieptepunten en waren meer dan eens degenen bij wie ik even mijn hart kon vitstorten. Ik ben er trots op dan jullie mijn paranimfen zijn.

A special word of thanks for my collaborators on this project in LA: Alan Stacy, Susan Ames, Jerry Grenard and Steve Sussman. Thanks for all your help with setting up the studies and writing the papers, but also for the wonderful time in LA. Alan, thanks for showing me the hospitality of your home, the beautiful Californian desert with the rocky off-road tracks and bush fires. Susan, thanks for all the special colorful moments and letting me feel right at home, but also for taking me out for the best Sushi in town and hanging out in Pasadena and China Town.

Mirjam Moerbeek, bedankt dat je me hebt kunnen laten zien hoe heerlijk helder statistiek kan zijn. Dit bracht me dit zoveel plezier, dat het proces er naar toe soms veel belangrijker leek dan de vitkomst zelf.

Dank aan de mensen die mee hebben gewerkt om deze studies in het veld van de grond te krijgen. Vrolijke Frans Feron, dank voor al je gezelligheid en hulp bij het opbouwen van een netwerk van scholen, waar we ons onderzoek konden uitvoeren. Dank aan Mirjam, Femke, Lotte, Lieke en Marten. Jullie hebben keihard meegewerkt aan dit project, waarin we soms in alle vroegte moest afreizen naar het viterste puntje van Limburg om drie scholieren te testen in een EHBO hok. Je een weg zoeken in de wereld van roosterwijzigingen, lesuitval, spijbelaars, proefwerken en overhoringen valt zeker niet mee. Zonder jullie had ik zeker niet al deze jongeren kunnen bereiken.

Tot slot, dank aan Guido en Maya. Jullie hebben me keer op keer laten zien dat het echte leven het meest waardevolle experiment van al is. 



\section{Curriculum Vitae}

Carolien Thush was born on November 27th 1979, in Haaksbergen, the Netherlands. In June 1998 she graduated from secondary school (Scholengemeenschap 'Het Assink', Haaksbergen). Immediately thereafter, she started studying Psychology at Maastricht University. During this period, she was actively involved in tutoring fellow students in psychology and statistics. In 2001, she interrupted her studies for one year and went traveling through Australia en Indonesia. Once returned, she graduated in January 2004 and received her master' degree in cognitive psychology. Directly following her graduation, she began her PhD research at the Department of Experimental Psychology, Maastricht University. She conducted parts of her research in collaboration with Prof. Dr. Alan Stacy at the University of Southern California. She currently holds a position as a post-doctoral fellow at the Department of Experimental Psychology, Maastricht University.

\section{PUBLICATIONS}

\section{International Publications}

Ames, S.L., Grenard, J., Thush, C., Sussman, S., Wiers, R. W. \& Stacy, A. W. (2007). Comparison of indirect assessments of marijuana-related associations among at-risk adolescents. Experimental Clinical Psychopharmacology 15, 204-218.

Grenard, J. L., Ames, S. L., Wiers, R. W., Thush, C., Stacy, A.W., \& Sussman, S. (2007). Brief intervention for substance use among at-risk adolescents: An initial study. Journal of Adolescent Health, 40, 188-191.

Grenard, J. L., Ames, S. L., Wiers, R. W., Thush, C., Sussman, S., \& Stacy, A.W. (in press).Working memory moderates the predictive effects of drug-related associations on substance use. Psychology of Addictive Behaviors.

Stewart, S., Conrod, P., Marlatt, A., Comeau, N., Thush, C. \& Krank, M. (2005). New developments in prevention and early intervention for alcohol abuse in youth. Alcoholism: Clinical and Experimental Research, 29, 278-286.

Thush, C., \& Wiers, R.W. (2007). Explicit and implicit alcohol-related cognitions and the prediction of future drinking in adolescents. Addictive Behaviors, 32, 1367 -1383.

Thush, C., Wiers, R.W. , Ames, S. L. , Grenard, J. L. , Sussman, S. , \& Stacy, A.W. (2007). Apples and oranges? Comparing indirect measures of alcohol-related cognition predicting alcohol use in at-risk adolescents. Psychology of Addictive Behaviors, 21, $587-591$. 
Thush, C., Wiers, R.W., Ames, S. L., Grenard, J. L., Sussman, S., \& Stacy, A.W. (2008). Interactions between implicit and explicit cognition and working memory in the prediction of alcohol use in at-risk adolescents. Drug and Alcohol Dependence, 94, $116-124$.

Thush, C. , Wiers, R.W. , Moerbeek, M., Ames, S. L. , Grenard, J. L. , Sussman, S. , \& Stacy, A.W. (in press). The influence of Motivational Interviewing on explicit and implicit alcohol-related cognition and alcohol use in at-risk adolescents. Psychology of Addictive Behaviors.

Thush, C., Wiers, R. W., Theunissen, N. , Van den Bosch, J. , Opdenacker, J., van Empelen, P., Moerbeek, M. \& F Feron, F. J. M. (2007). A randomized clinical trial of a targeted intervention to moderate alcohol use and alcohol-related problems in at-risk adolescents. Pharmacology, Biochemistry and Behavior, 86, 368 -376.

Van de Luitgaarden, J., Thush, C., Wiers, R. W., \& Knibbe, R. A. (2008). Prevention of alcohol problems in Dutch youth: Missed opportunities and new developments. Evaluation \& the Health Professions, 31, $167-181$.

Wiers, R. W., Bartholow, B. D., van den Wildenberg E., Thush C., Engels, R., Sher, K. J., Grenard, J. L., Ames, S. L. \& Stacy, A. W. (2007). Automatic and controlled processes and the development of addictive behaviors in adolescents: A review and a model. Pharmacology, Biochemistry and Behavior, 86, 263- 283.

\section{Chapters}

Wiers, R. W., Schoenmakers, T., Houben, K., Thush, C., Fadardi, J. S., Cox, W. M. (in press). Can problematic alcohol use be trained away? New behavioral treatments aimed at changing and moderating implicit cognitive processes in alcohol abuse. In C. R. Martin (Ed.). Identification and Treatment of Alcohol Dependency. UK: M\&K Publishing.

\section{National Publications}

Houben, K., Schoenmakers, T., Thush, C., \& Wiers, R. W. (2008). Impliciete cognitie en verslaving: Theoretische inzichten en praktische toepassingen. Gedragstherapie, 41, 169-183.

Thush, C., Wiers, R.W., Ames, S. L., Grenard, J. L., Sussman, S., \& Stacy, A.W. (2007). Appels en peren? Verschillende impliciete maten van alcoholgerelateerde cognitie als voorspellers van alcoholgebruik onder jongeren. In C. van Laar, R. Ruiter, J. Karremans, W. van Rijswijk, F. van Harreveld (Eds.), Jaarboek Sociale Psychologie. Groningen: ASPO Pers.

Thush, C., Wiers, R., van den Bosch, J., Opdenacker, J., Theunissen, N. \& Feron, F. (2004). 'Leren drinken'; Opzet en eerste resultaten van een preventieprogramma gericht op overmatig drinkende jongeren. Gedrag \& Gezondheid, 32, 193-202. 
Wiers, R.W. \& Thush, C. (2007). Zwaar drinkende jongeren: wat kunnen we doen en hoe krijgen we ze te pakken? Tijdschrift voor Gezondheidswetenschappen (TSG), 85(1), 63-65.

Wiers, R. W. \& Thush, C. (2007). Lessen achteraf of "I knew it all along" Tijdschrift voor Gezondheidswetenschappen (TSG) 85(2), 20-21.

Wiers, R. W. \& Thush, C. (2006). Secundaire preventie alcoholmisbruik onderzocht. PrePost, 8, $18-19$.

\section{Presentations}

Ames, S. L., Thush, C., Grenard, J. L., Wiers, R. W., Sussman, S. Y., \& Stacy, A. W. (2005). Translating basic research methods to field research in at-risk adolescent populations. Presentation given at the 113th Annual Meeting of the American Psychology Association (APA), Washington, USA.

Ames, S. L., Grenard, J. L., Thush, C., Sussman, S., Wiers, R.W., \& A. W. Stacy (2006). Comparison of indirect assessments of marijuana-related associations among atrisk adolescents. Poster presented at the $18 \mathrm{e}$ Annual Convention of the American Psychological Society (APS), New York, USA.

Grenard, J. L., Ames, S. L., Wiers, R.W., Thush, C, Sussman, S., \& A. W. Stacy (2006). Working memory interacts with implicit associations in the prediction of drug use. Poster presented at the 18e Annual Convention of the American Psychological Society (APS), New York, USA.

Thush, C. \& Wiers, R. W. (2005). Keeping track of changes: Explicit and implicit alcoholrelated cognition before and after the initiation of drinking. Poster presented at the Belgian Association for Psychological Science (BAPS) conference, Ghent, Belgium.

Thush, C. \& Wiers, R. W. (2005). Keeping track of changes: Implicit and explicit alcoholrelated cognition. Presentation given at the 28 e Annual Scientific Meeting of the de Research Society on Alcoholism (RSOA), Santa Barbara, USA.

Thush, C. \& Wiers, R. W. (2006). Using implicit alcohol- and drug-related cognitions to improve interventions in high-risk youth. Presentation given at the Pharmacology, Biochemistry and Behavior (PBB) conference entitled 'Adolescence: Alcohol, Drugs and Mental Disorders', Morzine, France.

Thush, C. \& Wiers, R. W. (2006). Reflex of Reflectie: Werkgeheugencapaciteit en impliciete associaties in de voorspelling van prospectief alcoholgebruik onder jongeren. Presentation given at the 16 th meeting of the Forum Alcohol en Drug Onderzoek (FADO), Utrecht, the Netherlands.

Thush, C. \& Wiers, R. W. (2006). Appels en peren? Verschillende impliciete maten van alcoholgerelateerde cognitie als voorspellers van alcoholgebruik onder jongeren. Presentation given at the annual conference of the Associatie van Sociaal Psychologische Onderzoekers (ASPO), Leiden, the Netherlands. 
Thush, C., Wiers, R.W., Ames, S. L., Grenard, J. L., Sussman, S., \& Stacy, A.W. (2006). Comparing implicit measures of alcohol-related cognition predicting alcohol use in at-risk adolescents. Poster presented at the 29e Annual Scientific Meeting of the Research Society on Alcoholism (RSOA), Baltimore, USA.

Thush, C., Wiers, R.W., Ames, S. L., Grenard, J. L., Sussman, S., \& Stacy, A.W. (2007). Reflex or reflect: Interaction between implicit and explicit cognition and working memory capacity in the prediction of prospective drinking in at-risk adolescents. Poster presented at the Adolescent Expert Meeting getiteld 'Risks and Opportunities in Adolescent Brain Development', Leiden, the Netherlands.

Thush, C., Wiers, R.W., Ames, S. L., Grenard, J. L., Sussman, S., \& Stacy, A.W. (2007). Reflex or reflect: Interaction between implicit and explicit cognition and working memory capacity in the prediction of prospective drinking in at-risk adolescents. Poster presented at the FWO Scientific Research Community meeting entitled 'Automatic processes in psychopathology and health related behavior', Ghent, Belgium.

Thush, C., Wiers, R.W., Ames, S. L., Grenard, J. L., Sussman, S., \& Stacy, A.W. (2008). Interactions between implicit cognition and working memory capacity in the prediction of alcohol use in at-risk adolescents. Presentation given at the 2008 Biennial Meeting of the Society for Research on Adolescence (SRA), Chicago, USA.

Thush, C., Wiers, R.W., Ames, S. L., Grenard, J. L., Sussman, S., \& Stacy, A.W. (2008). Reflex or reflect: Interaction between implicit and explicit cognition and working memory capacity in the prediction of prospective drinking in at-risk adolescents. Poster presented at the expert meeting 'Neurocognitive Approaches to Control and Working Memory', Leiden, the Netherlands.

Thush, C., Wiers, R. W, Theunissen, N., Opdenacker, J., Van den Bosch, J. \& Frans Feron (2004). A randomized clinical trial of a targeted prevention to moderate alcohol use and alcohol-related problems in adolescents at risk for alcoholism. Presentation given at the 27e Annual Scientific Meeting of the Research Society on Alcoholism (RSOA), Vancouver, Canada.

Wiers, R. W, Grenard, J. L., Thush, C. (2007). Executive functions moderate the effects of implicit associations to use alcohol and drugs. Presentation given at the World Behavior Therapy Conference, Barcelona, Spain.

Wiers, R. W., \& Thush, C. (2005). Using different versions of the EAST to assess alcohol and drug-related cognition. Presentation given at the EAST Workshop, Oppurg, Germany. 